\title{
Modelagem DE DAdos DE LONGA DURAÇÃO BASEADA EM PROCESSOS DE NASCIMENTO E MORTE LATENTES
}

\author{
Victor Silva Ritter
}

\author{
DisSERTAÇÃO APRESENTADA AO \\ Instituto DE MATEMÁTicA E EstatísticA \\ DA Universidade de SÃo PaUlo \\ PARA OBTENÇÃO DO TÍTULO DE \\ Mestre em CiÊnCias
}

Área de Concentração: Estatística

Orientador: Prof. Dr. Antonio Carlos Pedroso de Lima

Durante o desenvolvimento deste trabalho o autor

recebeu apoio financeiro do CNPq e da FAPESP

São Paulo, agosto de 2014 



\section{Modelagem DE DADOS DE LONGA DURAÇÃO BASEADA EM PROCESSOS DE NASCIMENTO E MORTE LATENTES}

Este exemplar corresponde à redação final da dissertação devidamente corrigida e defendida por Victor Silva Ritter, e aprovada pela Comissão Julgadora.

Comissão Julgadora:

- Prof. Dr. Antonio Carlos Pedroso de Lima (orientador) - IME, USP

- Prof ${ }^{a}$. Dra ${ }^{\mathrm{a}}$. Gisela Tunes da Silva - IME, USP

- Prof. Dr. Pranab Kumar Sen - UNC Chapel Hill, EUA 

"...as data becomes more complex, the data models become more cumbersome and are losing the advantage of presenting a simple and clear picture of nature's mechanism."

Breiman, L. (2001). Statistical modeling: The two cultures. Statistical Science 16(3), 199-231. 

Aos meus pais Verônica e Edson, ao meu irmão Tiago, à minha namorada Tamy e a minha amiga Tuany 



\section{Resumo}

Esse trabalho contribui com o desenvolvimento de um novo modelo para dados de sobrevivência com sobreviventes de longo termo visando uma formulação e interpretação mais realista do que a apresentada pelos modelos com fração de curados usuais. Motivados pelo estudo do tempo de sobrevivência residual para pacientes oncológicos, o modelo usa o processo de nascimento e morte para permitir a variação do

número de fatores de risco latentes durante um período precedente ao acompanhamento médico, considerando, então, um cenário de riscos competitivos para obtenção da função da sobrevivência (imprópria) dos pacientes. Simulações a aplicações à dados do Instituto do Câncer do Estado de São Paulo mostraram vantagens sobre o modelo de tempos de promoção.

Palavras-chave: análise de sobrevivência, fração de cura, termo longo, tempo de promoção, risco competitivo, tempo de vida residual, recidiva do câncer. 



\section{Abstract}

This work contributes with a new cure rate survival model developed aiming more realistic formulation and interpretations than the usual long-term survival models. Motivated by studying residual survival times in oncological patients, the model uses birth and death process to allow free variation on the number of latent risk factors during a pre-follow up period, then considers competing risks scenario for accessing the patients survival. Simulations and application to Instituto do Câncer do Estado de São Paulo data showed improvement over the promotion time model.

Keywords: survival analysis; cure rate; long-term; promotion time; competing risks; residual survival time; cancer relapse. 



\section{Agradecimentos}

Agradeço imensamente meu orientador, Antonio Carlos Pedroso de Lima, o qual vem acompanhando minha trajetória acadêmica desde a iniciação científica em 2010. É também desde esse período que o professor Antonio Carlos vem me dando conselhos importantes com relação às boas práticas de pesquisa, criticando sempre construtivamente meu trabalho, discutindo e alimentando horas de conversa sobre todo e qualquer tópico (pessoal ou profissional) levantado durante todos esses anos de trabalho. Sou grato por todas as maravilhosas oportunidades de crescimento acadêmico proporcionadas por ele, desde a elaboração de relatórios científicos, pôsteres e artigos até a oportunidade de pesquisa no exterior por ele intermediada. Sou grato não só à sua imensurável presteza e cuidado profissional durante às inúmeras revisões de trabalhos, muitas vezes feitas em extensas reuniões nas quais cada frase era discutida e comentada detalhadamente, mas também à sua enorme sensibilidade com relação ao meu bem estar, deixando claro a todo momento que valores como família e saúde devem prevalecer frente às questões profissionais, do contrário todo trabalho realizado será infértil. Sou grato ainda pela imensa ajuda e importante companhia durante os meses em que pudemos trabalhar juntos em Chapel-Hill, tendo sido esse período fundamental para meu amadurecimento acadêmico e pessoal.

Gostaria de agradecer em especial à professora Elisabeti Kira pelo excepcional trabalho realizado durante os três cursos por ela ministrados em que estive presente e por todo apoio prestado durante o período de pesquisa no exterior, não medindo esforços para fazer de minha primeira vivência internacional a melhor e mais agradável possível.

Agradeço também ao professor Julio da Motta Singer pelos inúmeros conselhos, consultorias e cursos ministrados, sempre de forma inteligente, objetiva e organizada. Agradeço também pelos inúmeros quilômetros pedalados, os quais certamente contribuíram para o sucesso desse e de outros trabalhos. 
Sou também imensamente grato ao professor Pranab Kumar Sen pela enorme dedicação durante o período de pesquisa em Chapel-Hill. Agradeço ao professor Sen por sua incrível didática, paciência e humildade, tratando questões e dúvidas muitas vezes básicas com igual ou maior importância que os mais elaborados conceitos estatísticos. Agradeço pelas inúmeras discussões realizadas durante as primeiras fazes desse trabalho, as quais foram fundamentais para a obtenção de um produto final satisfatório e de importância científica.

Agradeço a todos que indiretamente foram importantes para o bom desenvolvimento desse projeto, em especial destaco os funcionários da Diretoria do IME-USP, da Secretaria do Departamento de Estatística do IME-USP, da Fundação de Amparo à Pesquisa do Estado de São Paulo e da Gillings School of Global Public Health (UNC, Chapel-Hill)

Agradeço principalmente aos meus pais e ao meu irmão por terem me dado todo suporte emocional e material para que pudesse concluir mais uma etapa de minha formação. Sou imensamente grato à minha namorada Tamy pela paciência, companhia, ajuda e conselhos nas horas difíceis. Agradeço também a minha amiga Tuany pela ajuda durante a escrita desse trabalho, lembrando-me toda e qualquer regra gramatical existente.

Agradeço também ao Conselho Nacional de Pesquisa e Desenvolvimento por prover suporte financeiro durante os primeiros meses do mestrado no IME e pela Fundação de Amparo à Pesquisa do Estado de São Paulo pelo apoio financeiro no período restante do programa de mestrado e pela oportunidade de estágio no exterior totalmente financiada pela fundação.

Victor SiLVA RitTER

São Paulo, julho de 2014 


\section{Conteúdo}

$\begin{array}{ll}\text { Introdução } & 1\end{array}$

1 Conceitos iniciais $r$

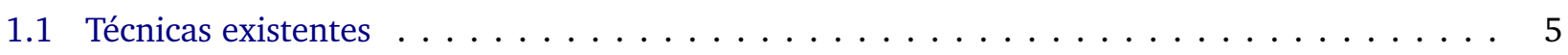

1.2 Processo de nascimento e morte $\ldots \ldots \ldots \ldots \ldots \ldots \ldots \ldots \ldots \ldots$

1.2 .1 Formulação geral $\ldots \ldots \ldots \ldots \ldots \ldots \ldots \ldots \ldots \ldots \ldots \ldots \ldots \ldots \ldots \ldots \ldots \ldots$

1.2 .2 Especificando as taxas de nascimento e morte $\ldots \ldots \ldots \ldots$

1.3 Modelo de tempos de promoção . . . . . . . . . . . . . . . . . . . . 14

1.3 .1 Formulação geral . . . . . . . . . . . . . . . . . . . . . 14

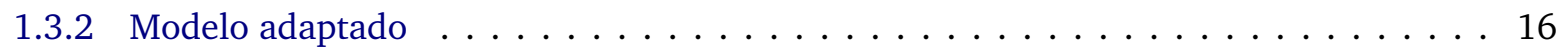

2 Abordagem proposta $\quad 21$

2.1 Cálculo da função de sobrevivência $\ldots \ldots \ldots \ldots \ldots \ldots \ldots \ldots$

2.2 Propriedades da função de sobrevivência $\ldots \ldots \ldots \ldots \ldots \ldots \ldots \ldots \ldots$

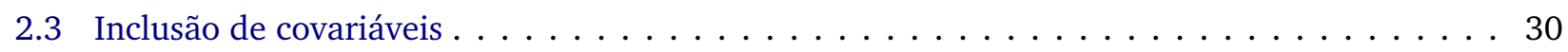

2.4 Verossimilhança e estimação . . . . . . . . . . . . . . . . . . 32

$\begin{array}{llr}3 & \text { Estudos de simulação } & 35\end{array}$

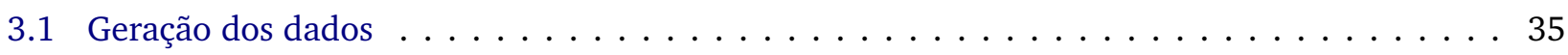

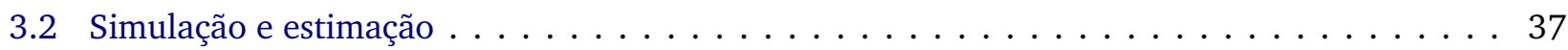

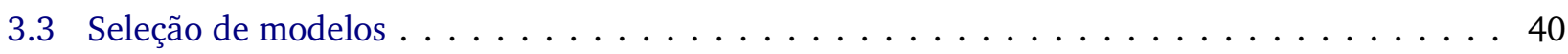

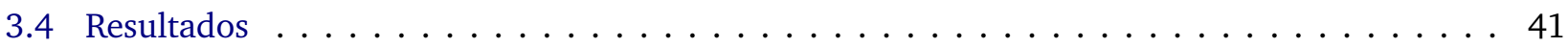


A Tabelas

51

B Gráficos 


\section{Introdução}

Metodologias que buscam reproduzir processos biológicos em sua formulação têm sido amplamente trabalhadas em análise de sobrevivência (Rodrigues et al. 2011; Rodrigues et al. 2009b; Tournoud \& Ecochard 2007; Tsodikov et al. 2003; Chen et al. 1999, entre outros), especialmente em estudos relacionados à área de oncologia, na qual fica evidente a importância de se considerar o mecanismo de evolução de um carcinoma na modelagem.

Em análise de sobrevivência, um dos principais modelos formulados com base em aspectos biológicos foi introduzido por Yakovlev et al. (1993) e denominado posteriormente modelo de tempos de promoção (ou tempos de progressão). Sua popularização deveu-se ao fato desse ser um modelo de fração de cura (também conhecido como modelo de longo termo), servindo como uma abordagem alternativa aos populares modelos de mistura introduzidos por Berkson \& Gage (1952). De fato, Rodrigues et al. (2009a) mostraram que ambos modelos podem ser representados de maneira comum, unificando, assim, a classe dos modelos de fração de cura em análise de sobrevivência.

Ao formular o modelo de tempos de promoção, Yakovlev et al. (1993) consideraram a modelagem do tempo até uma possível recidiva em pacientes tratados de câncer. Nesse tipo de estudo, após o término do tratamento, espera-se que uma expressiva fração dos pacientes apresente remissão do tumor e possam ser considerados livres da doença (indivíduos curados). Para os demais, assume-se que o número remanescente de células passíveis de gerar um novo tumor (denominadas clonogens, porém tratadas aqui simplesmente como carcinogens ou células cancerígenas) é aleatório com distribuição de Poisson. A cada uma dessas células cancerígenas é associada uma variável aleatória contínua positiva representando o tempo até o desenvolvimento de um novo tumor (denominado tempo de promoção ou tempo de progressão). Dessa forma, o tempo até a recidiva será dado pelo mínimo entre os tempos de remissão de cada célula, que 
usualmente são supostos independentes. A partir dessa estrutura é possível derivar a função de sobrevivência para cada paciente.

Apesar dessa modelagem estar fundamentada no processo biológico de crescimento tumoral, o modelo de tempos de promoção tem sido adotado em aplicações nas mais diversas áreas. Basta considerar a situação genérica em que indivíduos independentes são observados quanto ao tempo até a ocorrência de um evento de interesse; cada indivíduo apresenta um número aleatório de fatores latentes independentes, sendo que, a cada fator associa-se uma variável aleatória contínua positiva representado o tempo até àquele fator produzir o evento. Configura-se então um cenário de riscos competitivos no qual será observado apenas o menor dos tempos de sobrevivência, fazendo com que os demais tempos de promoção sejam latentes (não observáveis). Note que possibilitar um número de fatores latentes igual zero implica na existência de indivíduos curados, ou seja, indivíduos que não são suscetíveis ao evento de interesse.

Em finanças, por exemplo, pode-se considerar o tempo de sobrevivência como sendo o tempo até um cliente se tornar devedor e o número de fatores latentes como sendo o número de possíveis causas de fraude. Hoggart \& Griffin (2001) consideraram ainda a aplicação desse modelo para o estudo do tempo até o desligamento de um cliente, tendo como fatores latentes os eventos que podem levar o consumidor a encerrar suas atividades junto à empresa.

Na área da saúde, Tournoud \& Ecochard (2007) propuseram a utilização do modelo de tempos de promoção no contexto de doenças infectocontagiosas, sendo os fatores latentes o número de patógenos em contato com o indivíduo e o evento de interesse a detecção de uma doença infecciosa. Outra possível aplicação refere-se ao estudo do tempo até o retorno ao hospital após a alta médica, tendo como fatores latentes as possíveis complicações decorrentes de uma exposição ao ambiente hospitalar.

Buscando novamente motivação em estudos de câncer, Rodrigues et al. (2011) propuseram a introdução de uma nova característica biológica na modelagem. Denominado modelo de fração de cura Poisson ponderado destrutivo, os autores consideraram a possibilidade de que parte das células cancerígenas remanescentes fossem destruídas, representando o que ocorre durante tratamentos de irradiação ou simplesmente permitindo que ocorra morte celular.

Com o intuito aproximar ainda mais a abordagem teórica das características biológicas nos modelos com fração de curados, este trabalho se propõe a discutir e desenvolver uma metodologia que permita a livre 
variação do número de células cancerígenas segundo uma estrutura estocástica conveniente. Essa variação seria decorrente de processos biológicos como divisão e contaminação celular, os quais podem causar o aumento da população de células cancerosas, bem como de processos que levem à morte celular (natural ou por irradiação), contribuindo para a diminuição do tumor. Além disso, seria razoável considerar que a taxa com que o número de células malignas aumenta ou diminui varie com o tempo, pois usualmente a eficácia dos tratamentos aplicados à pacientes nesse quadro clínico tende a diminuir com o tempo.

Além da motivação teórica visando uma nova abordagem frente às técnicas existentes, também serviu de estímulo para esse trabalho o recorrente cenário prático em que pacientes oncológicos pós-operados permanecem um determinado período sob tratamento adjuvante. Nesses casos, em geral, há interesse em se estudar o tempo até uma possível recidiva ou o próprio tempo de sobrevida do paciente.

Há também um interesse especial em analisar dados provenientes do Instituto do Câncer do Estado de São Paulo (ICESP). O banco cedido pela instituição contém dados de 804 pacientes tratados contra diferentes tipos de carcinoma e acompanhados durante 2010 e 2012. Dentre as informações coletadas estão o tempo de sobrevida, tipo de procedimento adotado e tempo de permanência na unidade de tratamento intensivo (UTI). Pretende-se, então, ajustar o modelo proposto, discutir os resultados encontrados e comparar qualitativamente e quantitativamente a nova proposta com a de Yakovlev et al. (1993).

Assim como no modelo de tempos de promoção, diversas outras aplicações, além das na área de oncologia, podem ser idealizadas. Para tanto, basta adaptar os casos supracitados para o caso em que os fatores latentes podem variar ao longo do tempo. Nas aplicações em finanças, por exemplo, podemos considerar como período de tratamento a vigência de algum programa de fidelização adotado pela instituição financeira ou simplesmente um período fixo em que é observado um cenário financeiro específico. Na área da saúde, pode-se imaginar que o número de patógenos em contato com o indivíduo, ou ainda, que o número de fatores latentes no ambiente hospitalar, varia naturalmente em função da dinâmica dos sistemas biológicos envolvidos.

A metodologia apresentada nesse trabalho tem como principais objetivos:

- Introduzir uma nova técnica para análise de dados de longa duração que permita a variação do número de fatores latentes segundo um processo estocástico;

- Reproduzir mecanismos biológicos em sua formulação visando obter parâmetros interpretáveis e 
estimativas acuradas;

- Ser acessível aos demais pesquisadores e possuir aplicações práticas diversas à exemplo do modelo de tempos de promoção.

Para cumprir os objetivos propostos será adotado, em um primeiro momento, o enfoque paramétrico. Conforme salientado em Yakovlev \& Tsodikov (1996), essa abordagem permite: interpretação biológica clara dos parâmetros; possibilita a predição dos resultados para instantes além do período de acompanhamento, desde que feita da maneira criteriosa; e oferece meios simples para estimação da proporção de curados. Segundo o autor, essas vantagens acabam sobrepondo as restrições decorrentes da especificação de distribuição quando o modelo é suficientemente realista e produz resultados razoáveis. Além disso, o enfoque paramétrico possibilitará a otimização do processo de estimação, permitindo uma ampla exploração do modelo proposto.

Os conceitos necessários para a formulação da nova metodologia envolvendo análise de sobrevivência e processo de nascimento e morte são retomados nas seções que compõem o Capítulo 1. Em seguida, no Capítulo 2, é apresentado o modelo proposto e suas principais propriedades. O Capítulo 3 explica em detalhes os estudos de simulação conduzidos e apresenta os principais resultados. A aplicação do modelo aos dados do ICESP é apresentada no Capítulo 4, juntamente com a comparação entre a abordagem proposta e o modelo de tempos de promoção. Finalmente, no Capítulo 5 são discutidos alguns aspectos a respeito da metodologia desenvolvida e apresentados os próximos tópicos de pesquisa envolvendo o modelo proposto. 


\section{Capítulo 1}

\section{Conceitos iniciais}

Neste capítulo serão apresentadas algumas das metodologias motivadas por estudos oncológicos. Muitas dessas técnicas deixaram de ser trabalhadas devido à sua aplicabilidade restrita ou à falta de aparato computacional no período de seu desenvolvimento, porém serviram para o entendimento da dinâmica dos carcinomas e de base para a formulação das técnicas atualmente utilizadas.

Além disso, serão apresentadas as principais ferramentas utilizadas na formulação da nova metodologia. Assume-se que o leitor está familiarizado dos alguns conceitos básicos de análise de sobrevivência, processos estocásticos e regressão.

\subsection{Técnicas existentes}

A literatura a respeito da modelagem de dados provenientes da área de oncologia é vasta, incluindo estudos sobre crescimento tumoral, epidemiologia do câncer e ensaios clínicos relacionados à área de oncologia experimental. Em Yakovlev \& Tsodikov (1996) e Tan (2002) pode-se obter um completo histórico do tratamento estatístico quem vem sendo dado ao tema, contemplando os principais modelos, técnicas e aplicações existentes até as últimas décadas do século XX. Contendo abordagens mais recentes, Tsodikov et al. (2003) elaboram um interessante compilado das técnicas utilizadas na modelagem com fração de curados.

A modelagem de dados de câncer passou a despertar maior interesse de matemáticos e estatísticos a partir da década de 1950 com o surgimento de uma nova classe de modelos conhecida como modelos 
multi-estágio, introduzidos por Armitage \& Doll (1954) e amplamente estudada por Moolgavkar e colegas (Moolgavkar \& Venzon, 1979; Moolgavkar \& Knudson, 1981; Moolgavkar et al., 1988). Essa classe tem como principal representante o modelo de dois estágios, o qual acabou perdendo espaço após a década de 1990 para abordagens mais simples, como os modelos de mistura, de tempos de promoção, modelos Bayesianos e semiparamétricos.

Apesar de complexos, o apelo biológico dos modelos multi-estado ainda é destacável. Fazendo uso de processos Markovianos, os modelos dessa classe tentam reproduzir as diversas fases de desenvolvimento de um tumor (Tan, 1991). O modelo mais básico (dois estágios) considera que o câncer surge a partir de uma única célula tronco iniciada, ou seja, uma célula tronco cujo genótipo sofreu alteração devido à mutação, deleção, transcolação ou amplificação de sua carga genética; essa fase é denominada iniciação e pode ser influenciada por agentes ou substâncias externas denominados iniciadores. O próximo passo seria a reprodução descontrolada da célula iniciada gerando um tumor benigno; essa fase é denominada promoção e pode ser reversível caso não seja decorrente de alterações genéticas, mas sim da ação de fatores externos (promotores). O estágio final representa a evolução da massa cancerosa para um tumor maligno e a possível ocorrência de metástase; denominada progressão, essa fase depende da ocorrência de alterações genéticas específicas (Yang \& Chen, 1991).

A nomenclatura desses modelos corresponde ao número de alterações genéticas irreversíveis consideradas, dessa forma, o modelo com $k$-estágios considera a ocorrência de $k$ alterações genéticas na célula tronco primária e a progressão do tumor.

Diversas variações do modelo multi-estágio foram propostas. Klein \& Klein (1984) consideraram que o surgimento de um tumor maligno pode decorrer de diferentes trajetórias entre os estágios. Surgiu então a proposta de se considerar um modelo de múltiplos caminhos até a progressão do tumor; para tanto, dois ou mais modelos multi-estágio são anexados em paralelo. As diferenças entre indivíduos foram consideradas por Tan \& Singh (1987) no desenvolvimento dos modelos mistos de carcinogênese. Tan \& Chen (1995) consideraram, ainda, o surgimento de múltiplos tumores (múltiplos órgãos) na abordagem dos modelos multivariados de carcinogênese.

O elevado grau de detalhamento dos modelo multi-estágio acabou tornando essas metodologias interessantes teoricamente, porém pouco atraentes do ponto de vista prático. Conforme destacaram Tan \& Chen 
(1998), os modelos com três ou mais estágios são matematicamente intratáveis e os estados de seu processo marcoviano são não identificáveis (Moolgavkar \& Luebeck, 1992). Apenas o caso mais simples sob suposições restritivas pode ser trabalhado analiticamente, ainda assim o processo de estimação e ajuste para dados de câncer é extremamente complicado. Vale ressaltar que essas técnicas não têm foco em dados de sobrevivência, o que dificulta sua aplicação a dados censurados e em casos cuja presença de indivíduos curados parece razoável.

Introduzidos por Boag (1949) e popularizados sob a abordagem de mistura com Berkson \& Gage (1952), os modelos com fração de curados tiveram suas primeiras aplicações em oncologia com Yakovlev et al. (1993) e o modelo de tempos de promoção. A simplicidade e flexibilidade desses modelos proporcionou o desenvolvimento de diversas outras abordagens, inclusive da abordagem tratada nesse trabalho.

As técnicas envolvendo modelos com fração de curados até o final da década de 1990 tinham majoritariamente enfoque paramétrico clássico. A primeira abordagem Bayesiana para o modelo foi proposta por Chen et al. (1999), a qual desencadeou diversas outras técnicas com tratamento semelhante (Ibrahim et al., 2001; Yin \& Ibrahim, 2005; Kim et al., 2007 e outros). Apesar da grande popularidade do modelo semiparamétrico de Cox (Cox, 1972), foi apenas em Peng \& Dear (2000) que a primeira abordagem semiparamétrica para dados com fração de curados foi apresentada. Detalhes com relação a essa técnica podem ser encontrados em Peng (2003).

\subsection{Processo de nascimento e morte}

\subsubsection{Formulação geral}

Considere um processo estocástico em tempo contínuo $\{M(t), t \geq 0\}$, denotando o tamanho de uma população no tempo $t$. Para caracterizar a dinâmica dessa população assume-se a seguinte estrutura, para $t \geq 0$ :

$$
\begin{array}{lll}
\text { i. } & \mathbb{P}\{M(t+\Delta)=k+1 \mid M(t)=k\}=\lambda_{k}(t) \Delta+o(\Delta), & k=0,1, \ldots ; \\
\text { ii. } \quad & \mathbb{P}\{M(t+\Delta) \geq k+2 \mid M(t)=k\}=o(\Delta), & k=0,1, \ldots ; \\
\text { iii. } \quad & \mathbb{P}\{M(t+\Delta)=k-1 \mid M(t)=k\}=\mu_{k}(t) \Delta+o(\Delta), & k=1,2, \ldots ;
\end{array}
$$


iv. $\quad \mathbb{P}\{M(t+\Delta) \leq k-2 \mid M(t)=k\}=o(\Delta), \quad k=2,3, \ldots ;$

em que $\Delta$ é um incremento positivo, $\lambda_{k}(\cdot)$ e $\mu_{k}(\cdot)$ são funções positivas.

De acordo com (i)-(iv), $\lambda_{k}(\cdot)$ e $\mu_{k}(\cdot)$ controlam, respectivamente, as taxas de nascimento e morte da população. Além disso, a probabilidade de ocorrerem dois nascimentos (ou mortes) em um curto período de tempo torna-se negligível conforme o comprimento do intervalo considerado diminui.

A partir dessa estrutura deriva-se um sistema de equações diferenciais que permite a obtenção da expressão para a função geradora de probabilidades do processo de nascimento e morte. A seguinte notação será adotada para simplificar as expressões adiante:

$$
\mathbb{P}\left\{M\left(t_{2}\right)=j \mid M\left(t_{1}\right)=i\right\}=p_{i, j}\left(t_{1}, t_{2}\right),
$$

$\operatorname{com} t_{2}>t_{1}>0$ e $i, j=0,1, \ldots$

Para derivar o sistema assume-se a condição inicial $M(0)=i, i>0$ e avalia-se o processo $\{M(t)\}_{t \geq 0}$ nos extremos de dois intervalos consecutivos. Em seguida são consideradas todas as possíveis trajetórias que resultam na transição observada. Sem perda de generalidade, considere os intervalos $(0, t)$ e $(t, t+\Delta)$. Considerando todas as possíveis trajetórias de um estado $i$ para qualquer outro estado $j$ devemos observar um dos seguintes padrões:
a) $\quad\left\{m^{(0)}=i\right\} \rightarrow \cdots \rightarrow\left\{m^{(t)}=j\right\} \rightarrow \cdots \rightarrow\left\{m^{(t+\Delta)}=j\right\}$
b) $\quad\left\{m^{(0)}=i\right\} \rightarrow \cdots \rightarrow\left\{m^{(t)}=j-1\right\} \rightarrow \cdots \rightarrow\left\{m^{(t+\Delta)}=j\right\}$;
c) $\left\{m^{(0)}=i\right\} \rightarrow \cdots \rightarrow\left\{m^{(t)}=j-k\right\} \rightarrow \cdots \rightarrow\left\{m^{(t+\Delta)}=j\right\}$, $k=2, \ldots, j$
d) $\quad\left\{m^{(0)}=i\right\} \rightarrow \cdots \rightarrow\left\{m^{(t)}=j+1\right\} \rightarrow \cdots \rightarrow\left\{m^{(t+\Delta)}=j\right\}$;
e) $\quad\left\{m^{(0)}=i\right\} \rightarrow \cdots \rightarrow\left\{m^{(t)}=j+k\right\} \rightarrow \cdots \rightarrow\left\{m^{(t+\Delta)}=j\right\}, \quad k=2,3, \cdots$;

em que $m^{(s)}$ é o valor da trajetória observada no instante $s$ e os pontos representam quaisquer transições possíveis dentro dos intervalos considerados. Escrevendo as probabilidades de transição em termos dos padrões descritos teremos

$$
p_{i, j}(0, t+\Delta)=p_{i, j}(0, t) p_{j, j}(t, t+\Delta)+p_{i, j-1}(0, t) p_{j-1, j}(t, t+\Delta)+
$$




$$
\begin{aligned}
& +\sum_{k=2}^{j} p_{i, j-k}(0, t) p_{j-k, j}(t, t+\Delta)+p_{i, j+1}(0, t) p_{j+1, j}(t, t+\Delta)+ \\
& +\sum_{k \geq 2} p_{i, j+k}(0, t) p_{j+k, j}(t, t+\Delta) .
\end{aligned}
$$

Usando (ii) e (iv), pode-se escrever

$$
\begin{aligned}
p_{j, j}(t, t+\Delta)= & -\mathbb{P}\{M(t+\Delta) \neq j \mid M(t)=j\} \\
= & 1-(\mathbb{P}\{M(t+\Delta) \leq j-2 \mid M(t)=j\}+\mathbb{P}\{M(t+\Delta)=j-1 \mid M(t)=j\}+ \\
& \quad+\mathbb{P}\{M(t+\Delta)=j+1 \mid M(t)=j\}+\mathbb{P}\{M(t+\Delta) \geq j+2 \mid M(t)=j\}) \\
= & 1-\left(\lambda_{j}(t) \Delta+\mu_{j}(t) \Delta+o(\Delta)\right) \\
p_{j, j}(t, t+\Delta)= & 1-\left(\lambda_{j}(t)+\mu_{j}(t)\right) \Delta-o(\Delta) .
\end{aligned}
$$

Substituindo (1.3) em (1.2) e usando (i)-(iv), tem-se

$$
\begin{aligned}
p_{i, j}(0, t+\Delta)= & p_{i, j}(0, t)\left[1-\left(\lambda_{j}(t)+\mu_{j}(t)\right) \Delta-o(\Delta)\right]+p_{i, j-1}(0, t)\left[\lambda_{j-1}(t) \Delta+o(\Delta)\right]+ \\
& \quad+\sum_{k=2}^{j} p_{i, j-k}(0, t) o(\Delta)+p_{i, j+1}(0, t)\left[\mu_{j+1}(t) \Delta+o(\Delta)\right]+ \\
& +\sum_{k \geq 2} p_{i, j+k}(0, t) o(\Delta) \\
= & p_{i, j}(0, t)\left[1-\left(\lambda_{j}(t)+\mu_{j}(t)\right) \Delta\right]+p_{i, j-1}(0, t)\left[\lambda_{j-1}(t) \Delta\right]+ \\
& +p_{i, j+1}(0, t)\left[\mu_{j+1}(t) \Delta\right]+o(\Delta) .
\end{aligned}
$$

Logo,

$$
\begin{aligned}
\frac{p_{i, j}(0, t+\Delta)-p_{i, j}(0, t)}{\Delta}=- & p_{i, j}(0, t)\left(\lambda_{j-1}(t)+\mu_{j+1}(t)\right)+p_{i, j-1}(0, t) \lambda_{j-1}(t)+ \\
& +p_{i, j+1}(0, t) \mu_{j+1}(t)+\frac{o(\Delta)}{\Delta}
\end{aligned}
$$

Tomando o limite quando $\Delta \longrightarrow 0$ em (1.4), conclui-se que 


$$
\frac{\mathrm{d} p_{i, j}(0, t)}{\mathrm{d} t}=-p_{i, j}(0, t)\left(\lambda_{j}(t)+\mu_{j}(t)\right)+p_{i, j-1}(0, t) \lambda_{j-1}(t)+p_{i, j+1}(0, t) \mu_{j+1}(t),
$$

para $j \geq 1$. No caso em que $j=0$, (1.4) será

$$
\frac{p_{i, 0}(0, t+\Delta)-p_{i, 0}(0, t)}{\Delta}=-p_{i, 0}(0, t)\left(\lambda_{0}(t)+\mu_{0}(t)\right)+p_{i, 1}(0, t) \mu_{1}(t)+\frac{o(\Delta)}{\Delta} .
$$

Portanto

$$
\frac{\mathrm{d} p_{i, 0}(0, t)}{\mathrm{d} t}=-p_{i, 0}(0, t)\left(\lambda_{0}(t)+\mu_{0}(t)\right)+p_{i, 1}(0, t) \mu_{1}(t)
$$

Dessa forma, as probabilidades de transição $p_{i, j}(0, t)$ são completamente determinadas pelo sistema

$$
\left\{\begin{array}{l}
\frac{\mathrm{d} p_{i, j}(0, t)}{\mathrm{d} t}=-p_{i, j}(0, t)\left(\lambda_{j}(t)+\mu_{j}(t)\right)+p_{i, j-1}(0, t) \lambda_{j-1}(t)+p_{i, j+1}(0, t) \mu_{j+1}(t), \\
\frac{\mathrm{d} p_{i, 0}(0, t)}{\mathrm{d} t}=-p_{i, 0}(0, t)\left(\lambda_{0}(t)+\mu_{0}(t)\right)+p_{i, 1}(0, t) \mu_{1}(t)
\end{array}\right.
$$

e pelas condições iniciais

$$
p_{i, i}(0,0)=1 \quad \text { e } \quad p_{i, k}(0,0)=0, \quad \text { for } k \neq i
$$

Entretanto, (1.7) não pode ser resolvido diretamente. Chiang (1980) discute em detalhes as etapas necessárias para solução desse sistema. Porém, como o interesse aqui se restringe à expressão da função geradora de probabilidades, apenas serão aplicadas as expressões do sistema considerando alguma estrutura para as taxas de nascimento e morte.

\subsubsection{Especificando as taxas de nascimento e morte}

Considere agora uma estrutura básica para o processo, em que a taxa de nascimento $\lambda_{k}(t)$ e morte $\mu_{k}(t)$ são dadas por

$$
\lambda_{k}(t)=k \lambda(t) \quad \text { e } \quad \mu_{k}(t)=k \mu(t), \quad k=0,1, \ldots
$$


Assumindo $M(0)=m^{(0)}>0$, a função geradora de probabilidades desse processo será

$$
G_{M}(s, t)=\sum_{k=0}^{\infty} s^{k} p_{m^{(0)}, k}(0, t), \quad|s|<1, t \geq 0
$$

derivando termo a termo, obtem-se

$$
\begin{aligned}
\frac{\partial G_{M}(s, t)}{\partial t} & =\sum_{k=0}^{\infty} s^{k} \frac{\mathrm{d} p_{m^{(0)}, k}(0, t)}{\mathrm{d} t} \\
& =\frac{\mathrm{d} p_{m^{(0)}, 0}(0, t)}{\mathrm{d} t}+\sum_{k=1}^{\infty} s^{k} \frac{\mathrm{d} p_{m^{(0)}, k}(0, t)}{\mathrm{d} t}
\end{aligned}
$$

aplicando (1.7), segue que

$$
\begin{aligned}
\frac{\partial G_{M}(s, t)}{\partial t}=- & p_{m^{(0)}, 0}(0, t)\left(\lambda_{0}(t)+\mu_{0}(t)\right)+p_{m^{(0)}, 1}(0, t) \mu_{1}(t)+\sum_{k=1}^{\infty} s^{k}\left\{-p_{m^{(0), k}}(0, t)\left(\lambda_{k}(t)+\right.\right. \\
& \left.\left.+\mu_{k}(t)\right)+p_{m^{(0)}, k-1}(0, t) \lambda_{k-1}(t)+p_{m^{(0)}, k+1}(0, t) \mu_{k+1}(t)\right\}
\end{aligned}
$$

usando (1.8)

$$
\begin{aligned}
\frac{\partial G_{M}(s, t)}{\partial t}= & p_{m^{(0)}, 1}(0, t) \mu_{1}(t)+\sum_{k=1}^{\infty} s^{k}\left\{-k p_{m^{(0)}, k}(0, t)(\lambda(t)+\mu(t))+\right. \\
& \left.+(k-1) p_{m^{(0)}, k-1}(0, t) \lambda(t)+(k+1) p_{m^{(0)}, k+1}(0, t) \mu(t)\right\} \\
=- & (\lambda(t)+\mu(t)) \sum_{k=1}^{\infty} k s^{k} p_{m^{(0)}, k}(0, t)+\lambda(t) \sum_{k=1}^{\infty}(k-1) s^{k} p_{m^{(0)}, k-1}(0, t)+ \\
& +\mu(t) \sum_{k=0}^{\infty}(k+1) s^{k} p_{m^{(0)}, k+1}(0, t) \\
=- & s(\lambda(t)+\mu(t)) \frac{\partial G_{M}(s, t)}{\partial s}+s^{2} \lambda(t) \frac{\partial G_{M}(s, t)}{\partial s}+\mu(t) \frac{\partial G_{M}(s, t)}{\partial s} \\
= & (s-1)(s \lambda(t)-\mu(t)) \frac{\partial G_{M}(s, t)}{\partial s} .
\end{aligned}
$$

Portanto,

$$
\frac{\partial G_{M}(s, t)}{\partial t}+(1-s)(s \lambda(t)-\mu(t)) \frac{\partial G_{M}(s, t)}{\partial s}=0 .
$$

A obtenção da expressão para a função geradora requer a solução da equação diferencial parcial (1.10). 
Para uma revisão das principais técnicas de resolução o leitor pode recorrer a Chiang (1980). De acordo com essas técnicas, (1.10) pode ser resolvida a partir das equações auxiliares:

$$
\mathrm{d} G_{M}(s, t)=0 \quad \text { e } \quad \frac{\mathrm{d} t}{1}=\frac{\mathrm{d} s}{(1-s)(s \lambda(t)-\mu(t))} .
$$

Além disso, como a segunda equação em (1.11) é quadrática em $s$, Chiang (1980) sugere a mudança de variáveis $z=1 /(1-s)$, levando à equação auxiliar modificada

$$
\frac{\mathrm{d} t}{1}=\frac{\mathrm{d} z}{z(\lambda(t)-\mu(t))-\lambda(t)}
$$

a qual pode ser resolvida usando as técnicas regulares. Por exemplo, seja

$$
c(t)=\int_{0}^{t}(\mu(u)-\lambda(u)) \mathrm{d} u
$$

multiplicando (1.12) por $e^{c(t)}$ e rearranjando os termos, tem-se

$$
e^{c(t)} \frac{\mathrm{d} z}{\mathrm{~d} t}+z(\mu(t)-\lambda(t)) e^{c(t)}+\lambda(t) e^{c(t)}=0
$$

que corresponde a

$$
\frac{\mathrm{d}}{\mathrm{d} t}\left[z e^{c(t)}\right]+\lambda(t) e^{c(t)}=0
$$

Dessa forma, integrando (1.14) e retornando à variável original $s$, conclui-se que a solução geral da segunda equação auxiliar será dada por

$$
\frac{1}{1-s} e^{c(t)}+\int_{0}^{t} \lambda(u) e^{c(u)} \mathrm{d} u=c_{1}
$$

com $c_{1}$ sendo uma constante.

A solução da primeira equação auxiliar em (1.11) é trivialmente $G_{M}(s, t)=c_{2}$, sendo $c_{2}$ uma constante. Escrevendo $c_{2}$ em função de $c_{1}$ segue que

$$
G_{M}(s, t)=h\left\{\frac{1}{1-s} e^{c(t)}+\int_{0}^{t} \lambda(u) e^{c(u)} \mathrm{d} u\right\},
$$


em que $h(\cdot)$ é uma função diferenciável arbitrária. Usando a condição inicial para $t=0$, segue que

$$
h\left\{\frac{1}{1-s}\right\}=s^{m^{(0)}}
$$

Fazendo $v=1 /(1-s)$

$$
h(v)=\left\{1-\frac{1}{v}\right\}^{m^{(0)}}
$$

Portanto,

$$
G_{M}(s, t)=\left\{1-\left[\frac{1}{1-s} e^{c(t)}+\int_{0}^{t} \lambda(u) e^{c(u)} \mathrm{d} u\right]^{-1}\right\}^{m^{(0)}}
$$

$\operatorname{com} c(t)$ dado por (1.13).

Dependendo da especificação das taxas de nascimento e morte, (1.16) pode se tornar algebricamente intratável. Dessa forma, foi iniciado o desenvolvimento do novo modelo considerando o caso mais simples em que as taxas são constantes e, portanto, o crescimento e o decrescimento da população são lineares.

Sejam

$$
\lambda(t)=\lambda \quad \text { e } \quad \mu(t)=\mu, \quad \lambda, \mu \geq 0
$$

sendo necessário que ao menos uma das taxas seja não nula para que o processo esteja bem definido. Retomando a expressão da função geradora de probabilidades, (1.13) fica

$$
c(t)=(\mu-\lambda) t
$$

Logo, para $\lambda \neq \mu$, em (1.16) vem

$$
\int_{0}^{t} \lambda e^{c(u)} \mathrm{d} u=\frac{\lambda\left[e^{(\lambda-\mu) t}-1\right]}{\lambda-\mu} .
$$

Quando $\lambda=\mu, c(t)=0$, e assim,

$$
\int_{0}^{t} \lambda e^{c(u)} \mathrm{d} u=\int_{0}^{t} \lambda \mathrm{d} u=\lambda t
$$


Substituindo e realizando algumas manipulações algébricas, encontra-se

$$
G_{M}(s, t)= \begin{cases}{\left[\frac{\lambda t(1-s)+s}{\lambda t(1-s)+1}\right]^{m^{(0)}},} & \lambda=\mu \\ {\left[\frac{\lambda s-\mu+\mu(1-s) e^{(\lambda-\mu) t}}{\lambda s-\mu+\lambda(1-s) e^{(\lambda-\mu) t}}\right]^{m^{(0)}},} & \lambda \neq \mu\end{cases}
$$

para $|s|<1$ e $t \geq 0$.

\subsection{Modelo de tempos de promoção}

O modelo de tempos de promoção se mostrou especialmente importante no desenvolvimento da metodologia proposta nesse trabalho, pois, além de servir de escopo para sua derivação teórica, serviu como objeto de comparação. As seções que seguem tratam de sua derivação teórica, principais propriedades e métodos de estimação.

\subsubsection{Formulação geral}

Considere uma amostra aleatória de $n$ indivíduos tratados contra câncer sobre a qual deseja-se estudar o tempo até uma possível recidiva do tumor. Após o tratamento, cada indivíduo permanece com uma quantidade $M_{i}$ de células cancerígenas. Assumindo $M_{i}$ como sendo uma variável aleatória com distribuição de Poisson de média $\phi$, definem-se os tempos de sobrevivência (nesse caso, tempos de remissão) como sendo

$$
T_{i}= \begin{cases}\min \left\{V_{i 1}, V_{i 2}, \ldots, V_{i M_{i}}\right\}, & M_{i}>0 \\ +\infty, & M_{i}=0\end{cases}
$$

em que $V_{i j}$ é o tempo latente de remissão da $j$-ésima célula cancerígena remanescente no $i$-ésimo indivíduo, com $i=1, \ldots, n$ e $j=1, \ldots, M_{i}$. Dado $M_{i}=m_{i}$, consideram-se os tempos latentes independentes e identicamente distribuídos com função de distribuição $F_{V}(v ; \boldsymbol{\theta})=1-S_{V}(v ; \boldsymbol{\theta})$ a ser especificada. Além disso, a sequência $\left\{V_{i 1}, V_{i 2}, \ldots\right\}$ é suposta ser independente do número de fatores latentes $M_{i}$.

Note que a cardinalidade do conjunto de variáveis aleatórias do qual tomamos o mínimo é aleatória; dessa forma, deve-se trabalhar com a distribuição condicional dado que $M_{i}=m_{i}$. A função de sobrevivência 
individual pode então ser derivada fazendo-se

$$
\begin{aligned}
S_{T}(t ; \phi, \boldsymbol{\theta}) & =\sum_{m_{i}=0}^{\infty} \mathbb{P}\left\{T_{i}>t \mid M_{i}=m_{i}\right\} \mathbb{P}\left\{M_{i}=m_{i}\right\} \\
& =\mathbb{P}\left\{M_{i}=0\right\}+\sum_{m_{i}=1}^{\infty} \mathbb{P}\left\{V_{i 1}>t, \ldots, V_{i m_{i}}>t\right\} \mathbb{P}\left\{M_{i}=m_{i}\right\} \\
& =\sum_{m_{i}=0}^{\infty} S_{V}(t ; \boldsymbol{\theta})^{m_{i}} \mathbb{P}\left\{M_{i}=m_{i}\right\} \\
S_{T}(t ; \phi, \boldsymbol{\theta}) & =G_{M}\left(S_{V}(t ; \boldsymbol{\theta})\right) .
\end{aligned}
$$

Logo,

$$
S_{T}(t ; \phi, \boldsymbol{\theta})=\exp \left\{-\phi\left[1-S_{V}(t ; \boldsymbol{\theta})\right]\right\}
$$

A partir de (1.19) conclui-se que $S_{T}$ é imprópria, levando à uma proporção de curados correspondente à $\pi=e^{-\phi}$. Além disso, é fácil ver que as funções densidade e de risco associadas serão dadas por

$$
f_{T}(t ; \phi, \boldsymbol{\theta})=-\frac{\mathrm{d} S_{T}(t ; \phi, \boldsymbol{\theta})}{\mathrm{d} t}=\phi f_{V}(t ; \boldsymbol{\theta}) \exp \left\{-\phi\left[1-S_{V}(t ; \boldsymbol{\theta})\right]\right\}
$$

e

$$
h_{T}(t ; \phi, \boldsymbol{\theta})=\phi f_{V}(t ; \boldsymbol{\theta})
$$

Em geral, o modelo de tempos de promoção é adotado quando há interesse na modelagem da fração de curados. Dessa forma, a inclusão de covariáveis no caso de populações heterogêneas se dá por meio da atribuição de regressores à $\phi$ ou $\pi$.

É possível que os parâmetros da distribuição dos tempos latentes também recebam covariáveis, conforme explica Sposto (2002). De fato, Lambert et al. (2007) mostram que a modelagem incorreta desses parâmetros pode levar a estimativas enviesadas da proporção de cura. Nesse trabalho, foi considerado que apenas a proporção de cura varia de acordo com covariáveis, pois uma estrutura análoga foi proposta para o modelo em desenvolvimento. Adotou-se

$$
\log \phi_{i}=\eta_{i}, \quad \eta_{i}=\mathbf{x}_{i}^{\top} \boldsymbol{\beta}
$$


sendo $\mathbf{x}_{i}$ o vetor de $p$ covariáveis do i-ésimo indivíduo e $\boldsymbol{\beta}$ o vetor de parâmetros associados.

Com relação à função de ligação utilizada, optou-se pela logarítmica em detrimento de outras comumente utilizadas (ver Sposto, 2002) devido à sua simplicidade e conveniência durante o processo de estimação, possibilitando a avaliação irrestrita do vetor de parâmetros $\boldsymbol{\beta}$.

Para a construção da verossimilhança considera-se a possibilidade de censura aleatória dos tempos de sobrevivência. Seja, portanto, $Y_{i}=\min \left\{T_{i}, C_{i}\right\}$ o tempo de sobrevivência efetivamente observado para o i-ésimo paciente, em que $C_{i}$ e $T_{i}$ são, respectivamente, os tempos aleatórios de censura e de falha dados em (1.17). Seja ainda $\delta_{i}=\mathbb{1}_{\left\{T_{i} \geq C_{i}\right\}}$ o indicador de falha do paciente $i$. A verossimilhança será dada por

$$
\begin{aligned}
\mathcal{L}_{n}(\boldsymbol{\beta}, \boldsymbol{\theta} ; \mathbf{y}) & =\prod_{i=1}^{n} \mathcal{L}_{i}\left(\boldsymbol{\beta}, \boldsymbol{\theta} ; y_{i}\right) \\
& =\prod_{i=1}^{n} f_{T}\left(y_{i} ; \boldsymbol{\beta}, \boldsymbol{\theta}\right)^{\delta_{i}} S_{T}\left(y_{i} ; \boldsymbol{\beta}, \boldsymbol{\theta}\right)^{\left(1-\delta_{i}\right)} \\
\mathcal{L}_{n}(\boldsymbol{\beta}, \boldsymbol{\theta} ; \mathbf{y}) & =\prod_{i=1}^{n} h_{T}\left(y_{i} ; \boldsymbol{\beta}, \boldsymbol{\theta}\right)^{\delta_{i}} S_{T}\left(y_{i} ; \boldsymbol{\beta}, \boldsymbol{\theta}\right) .
\end{aligned}
$$

Logo, a log-verossimilhança é

$$
\begin{aligned}
& \log \mathcal{L}_{n}(\boldsymbol{\beta}, \boldsymbol{\theta} ; \mathbf{y})=\sum_{i=1}^{n}\left\{\delta_{i} \log h_{T}\left(y_{i} ; \boldsymbol{\beta}, \boldsymbol{\theta}\right)+\log S_{T}\left(y_{i} ; \boldsymbol{\beta}, \boldsymbol{\theta}\right)\right\} \\
& \log \mathcal{L}_{n}(\boldsymbol{\beta}, \boldsymbol{\theta} ; \mathbf{y})=\sum_{i=1}^{n}\left\{\delta_{i}\left(\mathbf{x}_{i}^{\top} \boldsymbol{\beta}+\log f_{V}\left(y_{i} ; \boldsymbol{\theta}\right)\right)-e^{\mathbf{x}_{i}^{\top} \boldsymbol{\beta}}\left(1-S_{V}\left(y_{i} ; \boldsymbol{\theta}\right)\right)\right\} .
\end{aligned}
$$

A estimação dos parâmetros $(\boldsymbol{\beta}, \boldsymbol{\theta})$ pode ser feita maximizando diretamente em (1.20) ou por meio do algoritmo EM (Chen \& Ibrahim, 2001). A Seção seguinte apresentará o modelo de tempos de promoção adaptado para a situação prática motivadora da metodologia proposta e tratará o processo de estimação em maiores detalhes.

\subsubsection{Modelo adaptado}

Como estudos discutidos anteriormente têm como objetivo estudar o tempo até a recidiva em pacientes pós-tratados para câncer, a principal característica do modelo adaptado é utilização de uma função de sobrevivência truncada no instante final do tratamento. Assim como no modelo usual, considera-se que 
o número de fatores latentes é fixo e que, portanto, a quantidade de células cancerígenas presentes no paciente permanecerá constante durante todo o período de tratamento. Claramente essa suposição não parece razoável, porém, adotá-la será importante para obtenção de um critério de comparação entre a técnica usual e a nova proposta.

Considere novamente um grupo de $n$ pacientes com câncer para os quais o interesse é estudar o tempo até uma possível recidiva, porém, dessa vez, suponha que cada paciente é submetido à cirurgia no instante $t=0$ e permanece em tratamento adjuvante até o instante $\xi_{i}$. Seja $M_{i}^{(0)}$ uma variável aleatória com distribuição de Poisson de média $\phi$ correspondente ao número de fatores latentes após a cirurgia. Assumindo que esse número não se altera durante o tratamento adjuvante, teremos $M_{i}^{\left(\xi_{i}\right)}$ (número de fatores latentes após o tratamento) equivalente à $M_{i}^{(0)}$, i.e.

$$
\mathbb{P}\left\{M_{i}^{(0)}=M_{i}^{\left(\xi_{i}\right)}\right\}=1
$$

Como antes,

$$
T_{i}= \begin{cases}\min \left\{V_{i 1}, V_{i 2}, \ldots, V_{\left.i M_{i}^{\left(\xi_{i}\right)}\right\},}\right. & M_{i}^{\left(\xi_{i}\right)}>0 \\ +\infty, & M_{i}^{\left(\xi_{i}\right)}=0\end{cases}
$$

novamente $V_{i j}$ é o tempo latente de remissão da $j$-ésima célula cancerígena remanescente no $i$-ésimo indivíduo, para $i=1, \ldots, n$ e $j=1, \ldots, M_{i}^{\left(\xi_{i}\right)}$. De maneira análoga ao modelo usual, dado $M_{i}^{\left(\xi_{i}\right)}=m_{i}^{\left(\xi_{i}\right)}$, os tempos latentes são considerados independentes e identicamente distribuídos com função de distribuição $F_{V}(v ; \boldsymbol{\theta})=1-S_{V}(v ; \boldsymbol{\theta})$ a ser especificada. Assume-se também que $\left\{V_{i 1}, V_{i 2}, \ldots\right\}$ é independente do número de fatores latentes $M_{i}^{\left(\xi_{i}\right)}$.

Considerando que todos os indivíduos permanecerão vivos durante o tratamento adjuvante, podemos definir

$$
V_{i j}=U_{i j}+\xi_{i}
$$

$\operatorname{com} U_{i j}>0$ variáveis aleatórias independentes com distribuição $F_{U}(u ; \boldsymbol{\theta})$. Portanto, a função de sobrevivência individual sará dada por

$$
S_{T}(t ; \phi, \boldsymbol{\theta})=\mathbb{P}\left\{T_{i}>t \mid T_{i}>\xi_{i}\right\}=\mathbb{1}_{\left\{0<t \leq \xi_{i}\right\}}+\frac{\mathbb{P}\left\{T_{i}>t\right\}}{\mathbb{P}\left\{T_{i}>\xi_{i}\right\}} \mathbb{1}_{\left\{t>\xi_{i}\right\}}
$$


procedendo com o cálculo das probabilidades de maneira análoga à (1.18) segue que

$$
S_{T}(t ; \phi, \boldsymbol{\theta})=\mathbb{1}_{\left\{0<t \leq \xi_{i}\right\}}+\exp \left\{-\phi\left[S_{V}\left(\xi_{i} ; \boldsymbol{\theta}\right)-S_{V}(t ; \boldsymbol{\theta})\right]\right\} \mathbb{1}_{\left\{t>\xi_{i}\right\}} \cdot
$$

Logo, as funções de distribuição e de risco associadas ao modelo adaptado são

$$
f_{T}(t ; \phi, \boldsymbol{\theta})=\phi f_{V}(t ; \boldsymbol{\theta}) \exp \left\{-\phi\left[S_{V}\left(\xi_{i} ; \boldsymbol{\theta}\right)-S_{V}(t ; \boldsymbol{\theta})\right]\right\} \mathbb{1}_{\left\{t>\xi_{i}\right\}}
$$

$\mathrm{e}$

$$
h_{T}(t ; \phi, \boldsymbol{\theta})=\phi f_{V}(t ; \boldsymbol{\theta}) \mathbb{1}_{\left\{t>\xi_{i}\right\}}
$$

De (1.21) conclui-se que a proporção de curados e o percentil de ordem $p(\times 100)$ do tempo de remissão no modelo adaptado são dados, respectivamente, por

$$
\pi=\exp \left\{-\phi S_{V}\left(\xi_{i} ; \boldsymbol{\theta}\right)\right\} \quad \text { e } \quad t_{p}=S_{V}^{-1}\left(S_{V}\left(\xi_{i}\right)+\frac{\log p}{\phi}\right)
$$

porém, o $(100 \times p)$-ésimo percentil estará bem definido apenas para $\pi<p$. Essas quantidades serão importantes para a comparação os modelos proposto e de tempos de promoção.

A atribuição de covariáveis e estimação do modelo segue de maneira análoga à formulação usual, fazendo

$$
\log \phi_{i}=\eta_{i}, \quad \eta_{i}=\mathbf{x}_{i}^{\top} \boldsymbol{\beta}
$$

sendo $\mathbf{x}_{i}$ o vetor de $p$ covariáveis do $i$-ésimo indivíduo e $\boldsymbol{\beta}$ o vetor $(p \times 1)$ de parâmetros associados. Novamente a adoção da função de ligação logarítmica é conveniente para estimação irrestrita do vetor de parâmetros $\boldsymbol{\beta}$.

Sejam, respectivamente, $Y_{i}=\min \left\{T_{i}, C_{i}\right\}$ e $\delta_{i}=\mathbb{1}_{\left\{T_{i} \leq C_{i}\right\}}$ o tempo de sobrevivência efetivamente observado e o indicador de falha para o $i$-ésimo paciente, com $C_{i}$ e $T_{i}$ os tempos aleatórios de censura e de falha. A verossimilhança do modelo adaptado será dada por

$$
\log \mathcal{L}_{n}(\boldsymbol{\beta}, \boldsymbol{\theta} ; \mathbf{y})=\sum_{i=1}^{n}\left\{\delta_{i}\left(\mathbf{x}_{i}^{\boldsymbol{\top}} \boldsymbol{\beta}+\log f_{V}\left(y_{i} ; \boldsymbol{\theta}\right)\right)-e^{\mathbf{x}_{i}^{\top} \boldsymbol{\beta}}\left(S_{V}\left(\xi_{i} ; \boldsymbol{\theta}\right)-S_{V}\left(y_{i} ; \boldsymbol{\theta}\right)\right)\right\}
$$


A estimação dos parâmetros do modelo adaptado foi feita a partir da maximização da log-verossimilhança (1.22) por meio do procedimento de Newton-Raphson (Atkinson, 2008). Para a obtenção do processo iterativo de maximização, considere $\boldsymbol{\psi}=(\boldsymbol{\beta}, \boldsymbol{\theta})$ o vetor $(p+q \times 1)$ de parâmetros e os vetores escore

$$
\underset{(p \times 1)}{\mathcal{U}_{\boldsymbol{\beta}}(\boldsymbol{\psi})}=\frac{\partial \log \mathcal{L}_{n}}{\partial \boldsymbol{\beta}} \quad \text { e } \quad \underset{(q \times 1)}{\mathcal{U}_{\boldsymbol{\theta}}(\boldsymbol{\psi})}=\frac{\partial \log \mathcal{L}_{n}}{\partial \boldsymbol{\theta}}
$$

A Informação de Fisher amostral observada será dada por

$$
\underset{(p+q \times p+q)}{\mathcal{I}_{F}(\boldsymbol{\psi})}=\left[\begin{array}{cc}
-\frac{\partial^{2} \log \mathcal{L}_{n}}{\partial \boldsymbol{\beta} \partial \boldsymbol{\beta}^{\top}} & -\frac{\partial^{2} \log \mathcal{L}_{n}}{\partial \boldsymbol{\beta} \partial \boldsymbol{\theta}^{\top}} \\
-\frac{\partial^{2} \log \mathcal{L}_{n}}{\partial \boldsymbol{\theta} \partial \boldsymbol{\beta}^{\boldsymbol{\top}}} & -\frac{\partial^{2} \log \mathcal{L}_{n}}{\partial \boldsymbol{\theta} \partial \boldsymbol{\theta}^{\top}}
\end{array}\right] .
$$

Considerando a expansão de Taylor do escore conjunto $\left(\mathcal{U}_{\boldsymbol{\beta}}, \mathcal{U}_{\boldsymbol{\theta}}\right)$ em torno do ponto $\boldsymbol{\psi}_{0}=\left(\boldsymbol{\beta}_{0}, \boldsymbol{\theta}_{0}\right)$ e utilizando o fato de que $\mathcal{U}_{\beta}(\widehat{\psi})=\mathcal{U}_{\theta}(\widehat{\psi})=0$ se $\widehat{\psi}$ é o estimador de máxima verossimilhança, segue que

$$
\left[\begin{array}{l}
\mathcal{U}_{\boldsymbol{\beta}}\left(\boldsymbol{\psi}_{0}\right) \\
\mathcal{U}_{\boldsymbol{\theta}}\left(\boldsymbol{\psi}_{0}\right)
\end{array}\right]=\mathcal{I}_{F}\left(\boldsymbol{\psi}_{0}\right)\left[\begin{array}{l}
\widehat{\boldsymbol{\beta}}-\boldsymbol{\beta}_{0} \\
\widehat{\boldsymbol{\theta}}-\boldsymbol{\theta}_{0}
\end{array}\right]+o_{p}(1)
$$

Baseado em (1.23), o algoritmo de Newton-Raphson considera o seguinte processo iterativo

$$
\left[\begin{array}{c}
\boldsymbol{\beta}_{k} \\
\boldsymbol{\theta}_{k}
\end{array}\right]=\left[\begin{array}{c}
\boldsymbol{\beta}_{k-1} \\
\boldsymbol{\theta}_{k-1}
\end{array}\right]+\left[\mathcal{I}_{F}\left(\boldsymbol{\psi}_{k-1}\right)\right]^{-1}\left[\begin{array}{l}
\mathcal{U}_{\boldsymbol{\beta}}\left(\boldsymbol{\psi}_{k-1}\right) \\
\mathcal{U}_{\boldsymbol{\theta}}\left(\boldsymbol{\psi}_{k-1}\right)
\end{array}\right]
$$

A partir de um valor inicial para os parâmetros, itera-se (1.24) até a convergência. Usualmente é adotado como critério de convergência a condição

$$
\left\|\psi_{k}-\psi_{k-1}\right\|<\varepsilon
$$

$\operatorname{com} \varepsilon$ uma constante positiva arbitrária. 



\section{Capítulo 2}

\section{Abordagem proposta}

Conforme discutido anteriormente, a metodologia proposta tem sua principal motivação na área de oncologia. Dessa forma, formularemos o modelo tendo como referência a seguinte situação prática: suponha um estudo em que pacientes com câncer são submetidos a um tratamento que consiste de uma intervenção cirúrgica seguida de um período pré-especificado de tratamento medicamentoso e/ou de irradiação; ao final desse período os pacientes são acompanhados quanto ao tempo de remissão do tumor. Como usual em estudos de análise de sobrevivência, possíveis perdas de informação devido a censuras devem ser sempre consideradas. Vale ressaltar que, apesar desse cenário servir de motivação para a formulação do modelo, suas aplicações não estão restritas a essa situação, conforme discutido na seção introdutória.

A Figura 2.1 ilustra as duas etapas consideradas na modelagem. Assim como no modelo de tempos de promoção, assume-se que após a intervenção cirúrgica (representada pelo instante $t=0$ no gráfico) um número $m_{i}^{(0)}(>0)$ de células cancerígenas permanece no indivíduo. O comportamento das células tumorais remanescentes passa então a ser regido por um processo de nascimento e morte, possibilitando a variação desse número de células ao longo do período de tratamento medicamentoso, o qual se estende até o instante pré especificado $\xi_{i}$. Nesse instante, considera-se que o número de células cancerígenas remanescentes é $m_{i}^{\left(\xi_{i}\right)}$.

Sendo o tratamento adjuvante efetivo no combate às células cancerígenas remanescentes, é razoável assumir que a taxa de nascimento seja menor que a taxa de morte e que, provavelmente, esse paciente deva experimentar uma diminuição do número de fatores latentes $\left(m_{i}^{(0)}>m_{i}^{\left(\xi_{i}\right)}\right)$. Eventualmente o paciente pode 
ser curado durante o tratamento $\left(m_{i}^{\left(\xi_{i}\right)}=0\right.$ ), ou seja, o processo de nascimento e morte atinge o estado absorvente zero. Por outro lado, a ineficácia do tratamento pode implicar em uma taxa de nascimento maior que a de morte e, portanto, um provável aumento do número de fatores latentes $\left(m_{i}^{(0)}<m_{i}^{\left(\xi_{i}\right)}\right)$. Caso o procedimento cirúrgico seja bem sucedido na remoção da massa tumoral, espera-se que $m_{i}^{(0)}$ seja pequeno, aumentando as chances de cura do paciente.

Ainda na Figura 2.1, dado o valor de $m_{i}^{\left(\xi_{i}\right)}$, a modelagem segue de maneira análoga ao modelo de tempos de promoção, utilizando o enfoque de riscos competitivos para a obtenção da função de sobrevivência imprópria do tempo de remissão. Nesse trabalho denomina-se a primeira fase da modelagem como fase de progressão ou fase de tratamento, e a fase seguinte como fase de promoção ou fase de acompanhamento.

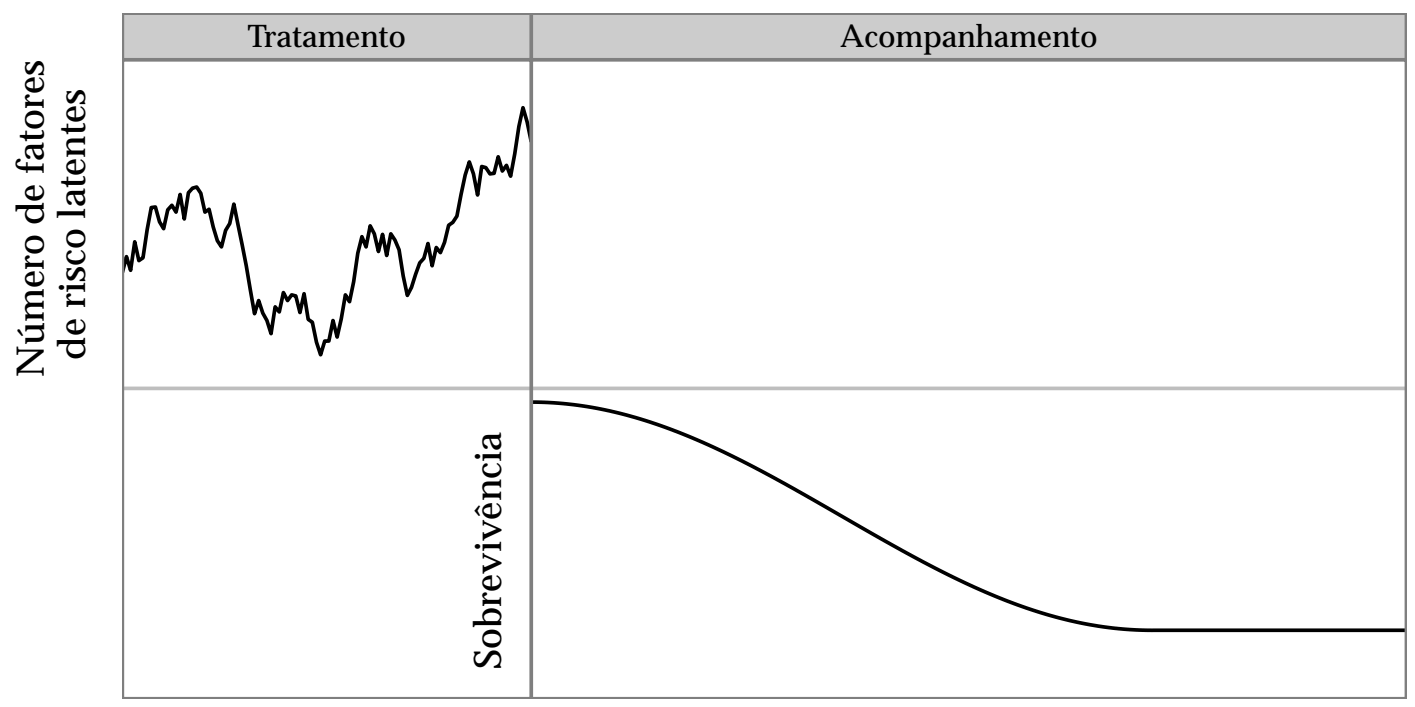

Tempo

Figura 2.1: representação das fases de tratamento e acompanhamento de um paciente hipotético. No período de tratamento observa-se a trajetória do número de fatores latentes (células cancerígenas) remanescentes. Após o tratamento, acompanha-se o indivíduo até a observação do evento de interesse (recidiva do câncer) ou da censura.

Uma formulação alternativa poderia considerar os processos de nascimento e morte evoluindo simultaneamente com o processo de promoção do tumor. Apesar dessa abordagem ter sido trabalhada, optou-se por tratar os dois processos separadamente, o que implicou em algumas restrições na aplicabilidade do modelo final; porém, serviu como passo inicial para o desenvolvimento da nova metodologia. Outra variação 
possível seria utilizar outro tipo de processo estocástico na modelagem do número de fatores latentes, entretanto, esse processo se mostrou bastante conveniente por apresentar uma formulação simples e de grande flexibilidade, podendo adequar-se às diversas características observadas na prática, como nos casos em que o tratamento não apresenta a eficácia esperada, ou perde sua eficiência ao longo do tempo.

\subsection{Cálculo da função de sobrevivência}

Suponha que $n$ pacientes são operados de câncer e, em seguida, submetidos a um tratamento complementar com duração de $\xi_{i}$ unidades de tempo. Considerou-se então o instante da cirurgia como início do estudo e durante o período de tratamento foi adotado o processo de nascimento e morte como mecanismo de modelagem do número de células cancerígenas. As derivações teóricas serão realizadas de maneira análoga ao modelo de tempos de promoção (ver Seção 1.3), utilizando o cenário de riscos competitivos.

Seja $\left\{M_{i}(t), t \geq 0\right\}$ um processo de nascimento e morte com taxas $\lambda_{k}=k \lambda$ e $\mu_{k}=k \mu$, sendo $\lambda, \mu>0$ e $k=0,1, \ldots$ Dessa forma, $M_{i}(t)$ representa o número de fatores latentes do $i$-ésimo indivíduo no instante $t$, com $i=1, \ldots, n$. Será considerada como condição inicial $M_{i}(0)=m_{i}^{(0)}>0, i=1, \ldots, n$, pois entende-se que, em um primeiro momento, todos os pacientes submetidos ao tratamento estão sujeitos ao evento de interesse, necessitando, portanto, de um número de fatores latentes estritamente maior que zero.

Fixado o término do tratamento em $t=\xi_{i}>0$, de maneira análoga ao modelo de tempo de promoção, define-se

$$
T_{i}= \begin{cases}\min \left\{V_{i 1}, V_{i 2}, \ldots, V_{i M_{i}\left(\xi_{i}\right)}\right\}, & M_{i}\left(\xi_{i}\right)>0 \\ +\infty, & M_{i}\left(\xi_{i}\right)=0\end{cases}
$$

em que $V_{i j}$ é novamente o tempo latente de remissão da j-ésima célula cancerígena remanescente no $i$-ésimo indivíduo, com $i=1, \ldots, n$ e $j=1, \ldots, M_{i}\left(\xi_{i}\right)$. Dado $M_{i}\left(\xi_{i}\right)=m_{i}^{\left(\xi_{i}\right)}$, consideram-se os tempos latentes independentes e identicamente distribuídos com função de distribuição $F_{V}(v ; \boldsymbol{\theta})=1-S_{V}(v ; \boldsymbol{\theta})$ a ser especificada. Além disso, supõe-se que a sequência $\left\{V_{i 1}, V_{i 2}, \ldots\right\}$ é independente do número de fatores latentes no instante $\xi_{i}$.

Considerando que o indivíduo permanece no estudo durante o tratamento e que, além disso, esteve livre da doença durante esse período, é razoável assumir que os tempos de promoção sejam truncados à 
esquerda no valor $\xi_{i}$, ou seja, $T_{i} \geq \xi_{i}$ para $i=1, \ldots, n$. Porém, considerando que a célula responsável pelo desencadeamento de um novo tumor pode ter surgido em qualquer instante durante o tratamento, a truncagem em $\xi_{i}$ passa a ser questionável.

Para contornar esse problema e preservar a simplicidade do modelo, os tempos latentes foram definidos como:

$$
V_{i j}=U_{i j}+(1-w) \xi_{i}, \quad i=1,2, \ldots, n
$$

com $U_{i j}>0$ uma variável aleatória com distribuição $F_{U}(u ; \theta)=1-S_{U}(u ; \theta)$ e $0 \leq w \leq 1$ uma constante conhecida. Note que, ao atribuir $w=0$, por exemplo, considera-se que a célula promotora do tumor esteve em remissão por pelo menos $\xi_{i}$, ao passo que com $w=1$ assume-se que a célula promovida teve seu surgimento momentos antes do final do período de tratamento. Dessa forma, $w$ pode ser interpretado como um fator de remissão, indicando que, quanto mas próximo de zero, mais as células cancerígenas são suscetíveis à remissão.

Definido o valor de $w$, a função de sobrevivência dos tempos latentes será dada por

$$
\begin{aligned}
S_{V}(v ; \boldsymbol{\theta}) & =\mathbb{P}\left\{V_{i}>v\right\} \\
& =\mathbb{P}\left\{U_{i}>v-(1-w) \xi_{i}\right\} \\
& =S_{U}\left(v-(1-w) \xi_{i} ; \boldsymbol{\theta}\right) \mathbb{1}_{\left\{v>(1-w) \xi_{i}\right\}}+\mathbb{1}_{\left\{v \leq(1-w) \xi_{i}\right\}},
\end{aligned}
$$

em que $\mathbb{1}_{\{x\}} A$ é 1 se $x \in A$ e zero caso contrário. Dado $M_{i}\left(\xi_{i}\right)=m_{i}^{\left(\xi_{i}\right)}$, tem-se a seguinte função de sobrevivência para o tempo de remissão individual

$$
S_{T_{i}}\left(t ; m_{i}^{(0)}, \lambda, \mu, \boldsymbol{\theta}\right)=\mathbb{P}\left\{T_{i}>t \mid T_{i}>\xi_{i}\right\}=\frac{\mathbb{P}\left\{T_{i}>t\right\}}{\mathbb{P}\left\{T_{i}>\xi_{i}\right\}}
$$

O numerador de (2.2) fica, para $t>\xi_{i}$,

$$
\begin{aligned}
\mathbb{P}\left\{T_{i}>t\right\} & =\sum_{m_{i}^{\left(\xi_{i}\right)}=0}^{\infty} \mathbb{P}\left\{T_{i}>t \mid M_{i}\left(\xi_{i}\right)=m_{i}^{\left(\xi_{i}\right)}\right\} \mathbb{P}\left\{M_{i}\left(\xi_{i}\right)=m_{i}^{\left(\xi_{i}\right)}\right\} \\
& =\mathbb{P}\left\{M_{i}\left(\xi_{i}\right)=0\right\}+\sum_{m_{i}^{\left(\xi_{i}\right)}=1}^{\infty} \mathbb{P}\left\{\min \left\{V_{i 1}, V_{i 2}, \ldots, V_{i M_{i}\left(\xi_{i}\right)}\right\}>t \mid M_{i}\left(\xi_{i}\right)=m_{i}^{\left(\xi_{i}\right)}\right\} \times
\end{aligned}
$$




$$
\begin{aligned}
& \times \mathbb{P}\left\{M_{i}\left(\xi_{i}\right)=m_{i}^{\left(\xi_{i}\right)}\right\} \\
= & \mathbb{P}\left\{M_{i}\left(\xi_{i}\right)=0\right\}+\sum_{m_{i}^{\left(\xi_{i}\right)}=1}^{\infty} \mathbb{P}\left\{\min \left\{V_{i 1}, V_{i 2}, \ldots, V_{\left.i m_{i}^{\left(\xi_{i}\right)}\right\}}\right\} t\right\} \mathbb{P}\left\{M_{i}\left(\xi_{i}\right)=m_{i}^{\left(\xi_{i}\right)}\right\} \\
= & \sum_{m_{i}^{\left(\xi_{i}\right)}=0}^{\infty} S_{V}(t ; \boldsymbol{\theta})^{m_{i}^{\left(\xi_{i}\right)}} \mathbb{P}\left\{M_{i}\left(\xi_{i}\right)=m_{i}^{\left(\xi_{i}\right)}\right\} \\
= & \sum_{m_{i}^{\left(\xi_{i}\right)}=0}^{\infty} S_{V}(t ; \boldsymbol{\theta})^{m_{i}^{\left(\xi_{i}\right)}} \sum_{m_{i}^{(0)}=1}^{\infty} \mathbb{P}\left\{M_{i}\left(\xi_{i}\right)=m_{i}^{\left(\xi_{i}\right)} \mid M_{i}(0)=m_{i}^{(0)}\right\} \mathbb{P}\left\{M_{i}(0)=m_{i}^{(0)}\right\} .
\end{aligned}
$$

Assumindo $m_{i}^{(0)}$ conhecido, segue que

$$
\mathbb{P}\left\{T_{i}>t\right\}=\sum_{m_{i}^{\left(\xi_{i}\right)}=0}^{\infty} S_{V}(t ; \boldsymbol{\theta})^{m_{i}^{\left(\xi_{i}\right)}} \mathbb{P}\left\{M_{i}\left(\xi_{i}\right)=m_{i}^{\left(\xi_{i}\right)} \mid M_{i}(0)=m_{i}^{(0)}\right\}
$$

Seguindo a notação introduzida em (1.1) e a definição (1.9) da função geradora de probabilidade do processo de nascimento e morte, conclui-se que

$$
\begin{aligned}
\mathbb{P}\left\{T_{i}>t\right\}= & G_{M}\left(S_{V}(t ; \boldsymbol{\theta}), \xi_{i}\right) \\
= & \begin{cases}{\left[\frac{\lambda \xi_{i}\left[1-S_{V}(t ; \boldsymbol{\theta})\right]+S_{V}(t ; \boldsymbol{\theta})}{\lambda \xi_{i}\left[1-S_{V}(t ; \boldsymbol{\theta})\right]+1}\right]^{m_{i}^{(0)}},} & \lambda=\mu ; \\
{\left[\frac{\lambda S_{V}(t ; \boldsymbol{\theta})-\mu+\mu\left[1-S_{V}(t ; \boldsymbol{\theta})\right] e^{(\lambda-\mu) \xi_{i}}}{\lambda S_{V}(t ; \boldsymbol{\theta})-\mu+\lambda\left[1-S_{V}(t ; \boldsymbol{\theta})\right] e^{(\lambda-\mu) \xi_{i}}}\right]^{m_{i}^{(0)}},} & \lambda \neq \mu ;\end{cases}
\end{aligned}
$$

para $t>\xi_{i}$.

Procedendo de maneira análoga para o denominador de (2.2) e denotando

$$
\frac{\lambda \xi_{i}\left[1-S_{V}(t ; \boldsymbol{\theta})\right]+S_{V}(t ; \boldsymbol{\theta})}{\lambda \xi_{i}\left[1-S_{V}(t ; \boldsymbol{\theta})\right]+1}=\gamma_{1}(t ; \boldsymbol{\lambda}, \boldsymbol{\theta})
$$

$\mathrm{e}$

$$
\frac{\lambda S_{V}(t ; \boldsymbol{\theta})-\mu+\mu\left[1-S_{V}(t ; \boldsymbol{\theta})\right] e^{(\lambda-\mu) \xi_{i}}}{\lambda S_{V}(t ; \boldsymbol{\theta})-\mu+\lambda\left[1-S_{V}(t ; \boldsymbol{\theta})\right] e^{(\lambda-\mu) \xi_{i}}}=\gamma_{2}(t ; \boldsymbol{\lambda}, \boldsymbol{\theta}),
$$

em que $\lambda$ é o vetor de taxas do processo de nascimento e morte $(\lambda=(\lambda, \mu)$ quando $\lambda \neq \mu$ e $\lambda=\lambda$ no caso 
em que $\lambda \neq \mu$ ), obtém-se a seguinte expressão para a função de sobrevivência do tempo até a recidiva

$$
S_{T_{i}}\left(t ; m_{i}^{(0)}, \boldsymbol{\lambda}, \boldsymbol{\theta}\right)=\left[\frac{\gamma_{j}(t ; \boldsymbol{\lambda}, \boldsymbol{\theta})}{\gamma_{j}\left(\xi_{i} ; \boldsymbol{\lambda}, \boldsymbol{\theta}\right)}\right]^{m_{i}^{(0)}}, \quad t \geq 0
$$

com $j=1$ no caso $\lambda=\mu$ e $j=2$ caso contrário. Considerando todo o período do estudo, teremos

$$
S_{T_{i}}\left(t ; m_{i}^{(0)}, \boldsymbol{\lambda}, \boldsymbol{\theta}\right)=\mathbb{1}_{\left\{0<t \leq \xi_{i}\right\}}+\left[\frac{\gamma_{j}(t ; \boldsymbol{\lambda}, \boldsymbol{\theta})}{\gamma_{j}\left(\xi_{i} ; \boldsymbol{\lambda}, \boldsymbol{\theta}\right)}\right]^{m_{i}^{(0)}} \mathbb{1}_{\left\{t>\xi_{i}\right\}}
$$

A expressão da sobrevivência (2.5) em si poderia ser útil na modelagem dos dados oriundos da situação prática apresentada anteriormente, porém, o fato do número de fatores latentes ter sido considerado conhecido pode dificultar a aplicação do modelo. Como solução para esse problema será utilizado o modelo marginalizado contendo apenas os parâmetros populacionais; dessa forma, preserva-se a simplicidade do modelo sem prejudicar sua aplicabilidade.

Suponha que, assim como no modelo de tempos de promoção, o número de fatores latentes iniciais tenha distribuição de Poisson com parâmetro $\rho>0$. Como restringimos $m_{i}^{(0)}>0$, considera-se a distribuição de Poisson truncada:

$$
\mathbb{P}\left\{M_{i}(0)=k\right\}=\frac{p_{k}}{1-e^{-\rho}}, \quad p_{k}=\frac{e^{-\rho} \rho^{k}}{k !} ; \quad k=1,2, \ldots
$$

A sobrevivência marginalizada, para $t>\xi_{i}$, será dada por

$$
\begin{aligned}
S_{T}(t ; \rho, \boldsymbol{\lambda}, \boldsymbol{\theta}) & =\sum_{k=1}^{\infty} S_{T_{i}}(t ; k, \boldsymbol{\lambda}, \boldsymbol{\theta}) \mathbb{P}\left\{M_{i}(0)=k\right\} \\
& =\sum_{k=1}^{\infty}\left\{\left[\frac{\gamma_{j}(t ; \boldsymbol{\lambda}, \boldsymbol{\theta})}{\gamma_{j}\left(\xi_{i} ; \boldsymbol{\lambda}, \boldsymbol{\theta}\right)}\right]^{k} \frac{p_{k}}{1-e^{-\rho}}\right\} \\
& =\frac{1}{1-e^{-\rho}}\left(\exp \left\{\rho\left[\frac{\gamma_{j}(t ; \boldsymbol{\lambda}, \boldsymbol{\theta})}{\gamma_{j}\left(\xi_{i} ; \boldsymbol{\lambda}, \boldsymbol{\theta}\right)}-1\right]\right\}-e^{-\rho}\right) \\
S_{T}(t ; \rho, \boldsymbol{\lambda}, \boldsymbol{\theta}) & =\frac{1}{e^{\rho}-1}\left(\exp \left\{\rho\left[\frac{\gamma_{j}(t ; \boldsymbol{\lambda}, \boldsymbol{\theta})}{\gamma_{j}\left(\xi_{i} ; \boldsymbol{\lambda}, \boldsymbol{\theta}\right)}\right]\right\}-1\right) .
\end{aligned}
$$


Logo, considerando $t>0$, tem-se

$$
S_{T}(t ; \rho, \boldsymbol{\lambda}, \boldsymbol{\theta})=\mathbb{1}_{\left\{0<t \leq \xi_{i}\right\}}+\frac{1}{e^{\rho}-1}\left(\exp \left\{\rho\left[\frac{\gamma_{j}(t ; \boldsymbol{\lambda}, \boldsymbol{\theta})}{\gamma_{j}\left(\xi_{i} ; \boldsymbol{\lambda}, \boldsymbol{\theta}\right)}\right]\right\}-1\right) \mathbb{1}_{\left\{t>\xi_{i}\right\}}
$$

em que as funções $\gamma_{j}(\cdot)$, com $j=1,2$, são dadas por (2.3) e (2.4), respectivamente.

\subsection{Propriedades da função de sobrevivência}

A primeira característica a se destacar com respeito à função de sobrevivência (2.6) certamente é o fato de sua expressão depender da relação entre as taxas de nascimento e morte. Como os casos $\lambda=\mu$ e $\lambda \neq \mu$ levam à expressões diferentes, será necessária a adoção de um critério para escolha entre os dois submodelos possíveis.

A princípio foi trabalhada a elaboração de um teste estatístico para a hipótese de igualdade entre as taxas baseado na razão de verossimilhanças, porém, as inconsistências apresentadas em estudos de simulação realizados acabou levando à tomada de decisões baseadas em critérios de informação (Akaike, 1974; Schwarz, 1978) e reamostragens bootstrap (Efron, 1979).

Dada a relação entre as taxas de nascimento e morte, verifica-se que de fato $S_{T}(\cdot)$ é uma função de sobrevivência imprópria. Considere as funções auxiliares

$$
\begin{array}{cl}
f(x)=\frac{e^{\rho x}-1}{e^{\rho}-1}, & 0 \leq x \leq 1, \rho>0, \\
g_{1}(x)=\frac{\lambda \xi_{i}(1-x)+x}{\lambda \xi_{i}(1-x)+1}, & 0 \leq x \leq 1, \xi_{i}>0, \lambda>0, \\
g_{2}(x)=\frac{\lambda x-\mu+\mu(1-x) e^{(\lambda-\mu) \xi_{i}}}{\lambda x-\mu+\lambda(1-x) e^{(\lambda-\mu) \xi_{i}},} & 0 \leq x \leq 1, \xi_{i}>0, \lambda>0, \\
h(x)=S_{V}(x ; \theta), & \mu>0, \lambda \neq \mu, \\
& x \geq \xi_{i} .
\end{array}
$$

Portanto, pode-se escrever $S_{T}$ por meio da composição

$$
S_{T}(x ; \rho, \lambda, \boldsymbol{\theta})=\left(f \circ g_{j} \circ h\right)(x), \quad x \geq \xi_{i}, j=1,2 .
$$


Para verificar que (2.6) é não-crescente toma-se sua derivada

$$
\left(f \circ g_{j} \circ h\right)^{\prime}(x)=\left[f^{\prime} \circ\left(g_{j} \circ h\right)(x)\right]\left[\left(g_{j}^{\prime} \circ h\right)(x)\right] h^{\prime}(x)
$$

em que

$$
\begin{array}{cl}
f^{\prime}(x)=\frac{\rho e^{\rho x}}{e^{\rho}-1}, & 0 \leq x \leq 1, \rho>0, \\
g_{1}^{\prime}(x)=\frac{1}{\left(\lambda \xi_{i}(1-x)+1\right)^{2}}, & 0 \leq x \leq 1, \xi_{i}>0, \lambda>0, \\
g_{2}^{\prime}(x)=\frac{(\lambda-\mu)^{2} e^{(\lambda-\mu) \xi_{i}}}{\left[\lambda x-\mu+\lambda(1-x) e^{(\lambda-\mu) \xi_{i}}\right]^{2}}, & 0 \leq x \leq 1, \xi_{i}>0, \lambda>0, \\
h^{\prime}(x)=-f_{V}(x ; \boldsymbol{\theta}), & \mu>0, \lambda \neq \mu, \\
& x \geq \xi_{i} .
\end{array}
$$

Logo, como $f^{\prime} \circ\left(g_{j} \circ h\right)(x)>0,\left(g_{j}^{\prime} \circ h\right)(x)>0$ e $h^{\prime}(x) \leq 0$, segue que a derivada de $S_{T}$ é estritamente negativa, o que verifica sua monotonicidade não-crescente.

A proporção de curados será determinada pelo comportamento da função de sobrevivência quando $t$ tende a infinito. Como $S_{V}(\cdot)$ é própria, segue

$$
\begin{aligned}
& \lim _{t \rightarrow \infty} \frac{\gamma_{1}(t ; \lambda, \boldsymbol{\theta})}{\gamma_{1}\left(\xi_{i} ; \lambda, \boldsymbol{\theta}\right)}=\frac{\lambda \xi_{i}}{\lambda \xi_{i}+1} \gamma_{1}\left(\xi_{i} ; \lambda, \boldsymbol{\theta}\right)^{-1}=q_{1}, \\
& \lim _{t \rightarrow \infty} \frac{\gamma_{2}(t ; \boldsymbol{\lambda}, \boldsymbol{\theta})}{\gamma_{2}\left(\xi_{i} ; \boldsymbol{\lambda}, \boldsymbol{\theta}\right)}=\frac{\mu e^{(\lambda-\mu) \xi_{i}}-\mu}{\lambda e^{(\lambda-\mu) \xi_{i}}-\mu} \gamma_{2}\left(\xi_{i} ; \boldsymbol{\lambda}, \boldsymbol{\theta}\right)^{-1}=q_{2} .
\end{aligned}
$$

Para verificar que $0 \leq q_{j} \leq 1, j=1,2$, pode-se argumentar que a monotonicidade não-crescente das funções $\gamma_{j}$ é condição suficiente. Utilizando as funções auxiliares definidas anteriormente, tem-se que $\gamma_{j}(x ; \boldsymbol{\lambda}, \boldsymbol{\theta})=\left(g_{j} \circ h\right)(x)$, para $x \geq \xi_{i} ;$ portanto, $\gamma_{j}^{\prime}(x ; \boldsymbol{\lambda}, \boldsymbol{\theta})=\left[\left(g_{j}^{\prime} \circ h\right)(x)\right] h^{\prime}(x)$. Vimos que $\left[\left(g_{j}^{\prime} \circ h\right)(x)\right]>0$ e $h^{\prime}(x) \leq 0$; logo, segue a monotonicidade não-crescente de $\gamma_{j}$ e, consequentemente, o fato de que $q_{1}$ e $q_{2}$ são proporções.

Dessa forma, a proporção de curados será dada por

$$
\pi_{j}=\frac{e^{\rho q_{j}}-1}{e^{\rho}-1}, \quad j=1,2
$$


com $q_{j}$ dado por (2.7) e (2.8).

A partir de (2.6), pode-se obter o percentil $100 \times p$ do tempo de remissão. Fazendo $S_{T}\left(t_{p} ; \rho, \lambda, \boldsymbol{\theta}\right)=p$, para $p>\pi_{j}$, segue que

$$
\gamma_{j}\left(t_{p} ; \lambda, \boldsymbol{\theta}\right)=u_{1}, \quad u_{1}=\frac{1}{\rho} \gamma_{j}\left(\xi_{i} ; \lambda, \boldsymbol{\theta}\right) \log \left\{p\left(e^{\rho}-1\right)+1\right\}
$$

Logo, a partir das expressões (2.3) e (2.4), tem-se $S_{V}\left(t_{p} ; \boldsymbol{\theta}\right)=u_{2 j}$, em que

$$
\begin{array}{cc}
u_{21}=\frac{\lambda \xi_{i}\left(1-u_{1}\right)+u_{1}}{\lambda \xi_{i}\left(1-u_{1}\right)+1}, & \lambda=\mu ; \\
u_{22}=\frac{\mu\left(1-u_{1}\right)+\left(\lambda u_{1}-\mu\right) e^{(\lambda-\mu) \xi_{i}}}{\lambda\left(1-u_{1}\right)+\left(\lambda u_{1}-\mu\right) e^{(\lambda-\mu) \xi_{i}}} & \lambda \neq \mu ;
\end{array}
$$

Finalmente os percentis do tempo de remissão serão dados por

$$
t_{p}=S_{V}^{-1}\left(u_{2 j} ; \boldsymbol{\theta}\right)
$$

A Figura 2.2 ilustra o comportamento da função de sobrevivência quando variados os valores das taxas de nascimento e morte. Como esperado, o aumento da taxa de nascimento mantendo-se fixos os demais parâmetros acarreta a diminuição da proporção de curados, ou seja, quanto maior a taxa com que surgem carcinogens, maior a chance de recidiva. Procedendo da mesma forma para a taxa de morte, seu aumento produz a elevação da fração de cura, indicando que o tratamento deve ser efetivo na remissão do tumor.

Na situação em que as taxas de nascimento e morte são iguais, representando o caso de um paciente estável, sugere-se a interpretação dessa quantidade como a taxa de instabilidade dos carcinogens, pois quanto maior seu valor, maior o número de transições por intervalo de tempo fixado no processo de nascimento e morte. Nesse caso, a Figura 2.3 exemplifica o comportamento da função de sobrevivência mediante o aumento dessa taxa, ocasionando o aumento da proporção de curados. Esse comportamento pode ser explicado pelo fato de que, sendo o processo mais instável, maior é a probabilidade haja a transição para o estado absorvente zero (inexistência de fatores de risco), implicando na cura do paciente. 


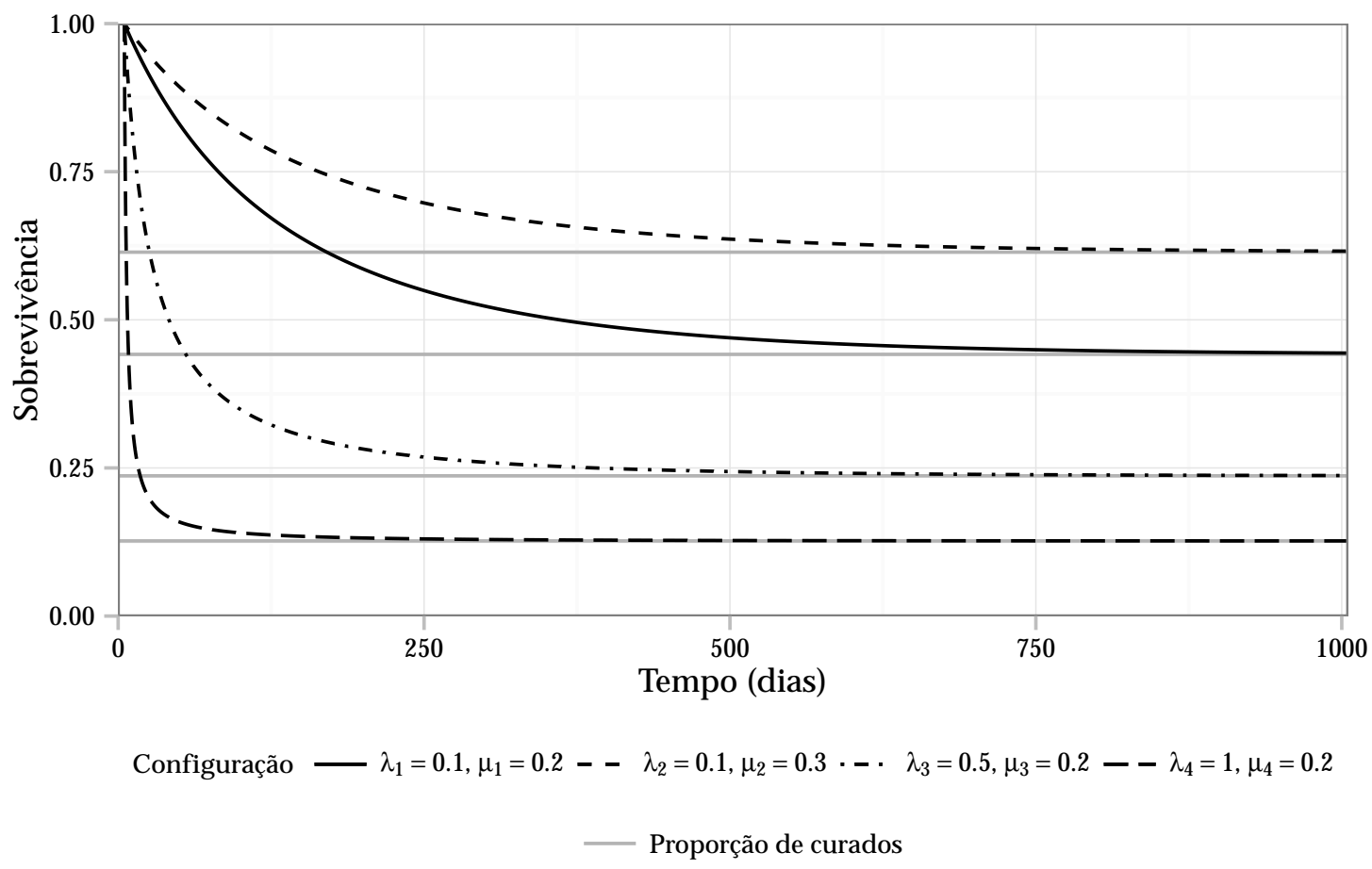

Figura 2.2: efeito da variação das taxa de nascimento e morte na função de sobrevivência quando $\lambda \neq \mu$.

\subsection{Inclusão de covariáveis}

Um dos principais interesses na aplicação do modelo é a comparação de grupos submetidos a diferentes tratamentos e detecção dos efeitos das covariáveis no tempo de remissão e na proporção de curados. Sendo assim, parece razoável que os regressores estejam associados às taxas de nascimento e morte, uma vez que são esses os parâmetros responsáveis pelo comportamento da população de células tumorais.

Considerando primeiramente o caso mais simples em que $\lambda=\mu$, seja $\mathbf{x}_{i}$ o vetor das $p$ covariáveis do indivíduo $i$ e $\boldsymbol{\beta}$ o vetor de parâmetros desconhecidos associado. Propõe-se

$$
\log \lambda_{i}=\eta_{i}
$$

$\operatorname{com} \eta_{i}=\mathbf{x}_{i}^{\top} \boldsymbol{\beta}$. Dessa forma, $\boldsymbol{\beta}$ pode ser estimado de maneira irrestrita.

A interpretação do vetor de parâmetros $\beta$ pode ser feita em termos da razão entre as taxas de instabilidade. Tomando, por exemplo, $\eta_{i}=\beta x_{i}$ e dois indivíduos com covariáveis $x_{1}=x$ e $x_{2}=x+1$ obtêm-se que $\lambda_{2}=e^{\beta} \lambda_{1}$, ou seja, a taxa de instabilidade do paciente 2 é $e^{\beta}$ vezes a taxa de instabilidade do paciente 1 . 


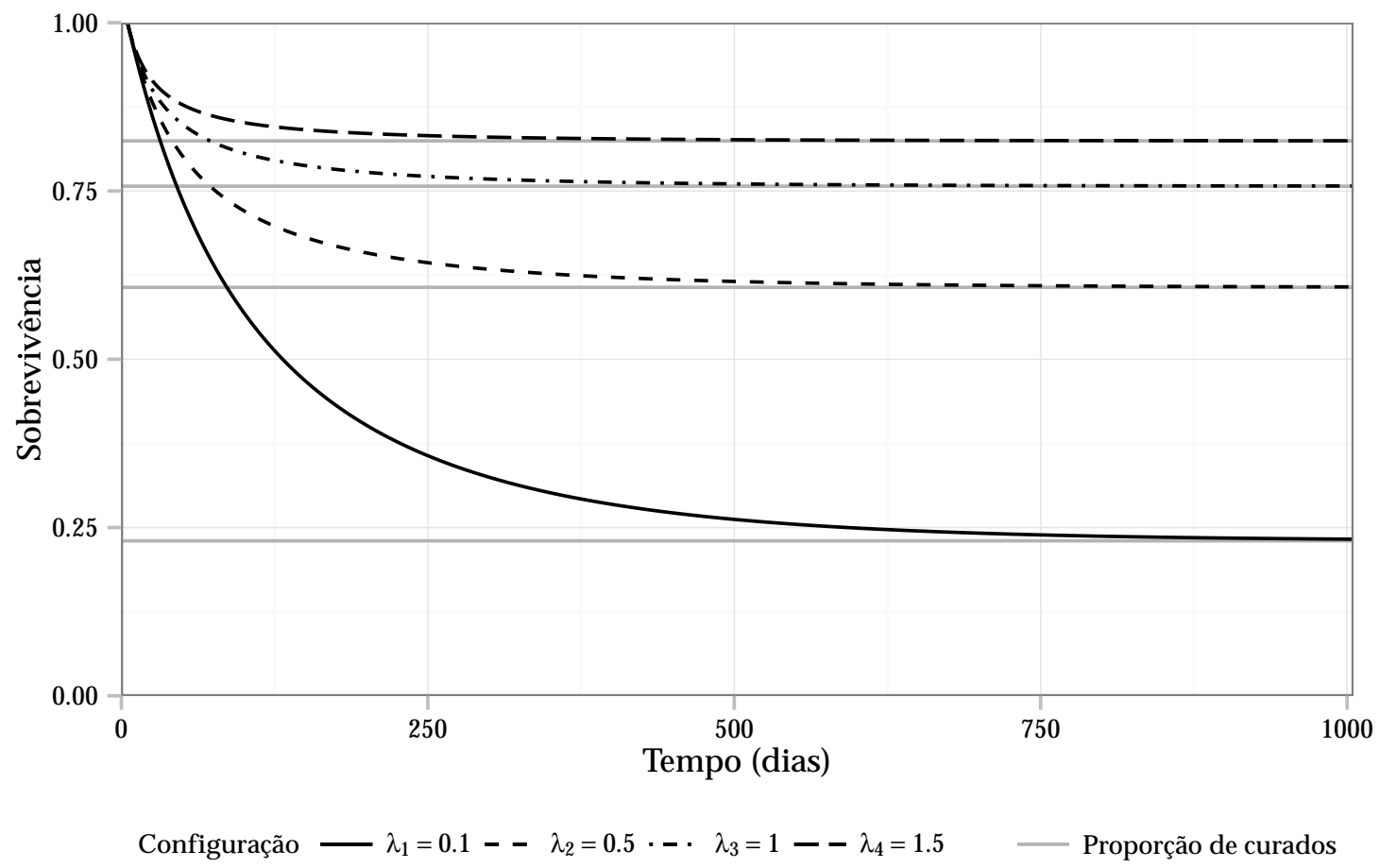

Figura 2.3: efeito da variação das taxa de nascimento e morte na função de sobrevivência quando $\lambda=\mu$.

No caso em que $\lambda \neq \mu$, foi observada uma grande instabilidade nas estimativas para as taxas de morte, o que levou à adoção de uma relação funcional entre as taxas de nascimento e morte. Considerou-se, então

$$
\log \lambda_{i}=\eta_{i} \quad \text { e } \quad \log \mu_{i}=g\left(\eta_{i}\right)
$$

em que $\eta_{i}$ é novamente o regressor linear e $g(\cdot)$ uma função determinística.

Em virtude da arbitrariedade da relação funcional entre as taxas de nascimento e morte, o procedimento de seleção de modelos baseado em critérios de informação e reamostragens comentado no início da Seção 2.2 foi estendido para auxiliar na decisão acerca da relação funcional a ser adotada (ver Capítulo 3).

Dessa forma, o vetor de parâmetros $\boldsymbol{\beta}$ estará, portanto, associado tanto à taxa de nascimento quanto à de morte. Logo, sua interpretação deve ser em termos de ambas as taxas. Suponha que valem as propriedades distributivas para a função $g$, nesse caso, podemos interpretar $\boldsymbol{\beta}$ em termos de razões entre taxas de nascimento e $\boldsymbol{\beta}^{*}=g(\boldsymbol{\beta})$ por meio de razões entre taxas de morte. 


\subsection{Verossimilhança e estimação}

Para a construção da verossimilhança, será considerada a perda parcial de informação devido à censura. Seja $Y_{i}=\min \left\{T_{i}, C_{i}\right\}$ o tempo de sobrevivência efetivamente observado para o $i$-ésimo indivíduo, no qual $C_{i}$ e $T_{i}$ são respectivamente o tempo de censura aleatória e o tempo de sobrevivência dado por (2.1). Define-se também o indicador de censura $\delta_{i}=\mathbb{1}_{\left\{T_{i} \leq C_{i}\right\}}$.

Para obter a expressão da log-verossimilhança, considere inicialmente o logaritmo das funções de sobrevivência e densidade. Tem-se que

$$
\log S_{T}\left(y_{i} ; \rho, \boldsymbol{\beta}, \boldsymbol{\theta}\right)=\log \left(\exp \left\{\rho\left[\frac{\gamma_{j}(t ; \boldsymbol{\beta}, \boldsymbol{\theta})}{\gamma_{j}\left(\xi_{i} ; \boldsymbol{\beta}, \boldsymbol{\theta}\right)}\right]\right\}-1\right)-\log \left(e^{\rho}-1\right), \quad j=1,2
$$

Fazendo uso das funções auxiliares, segue

$$
\log f_{T}\left(y_{i} ; \rho, \boldsymbol{\beta}, \boldsymbol{\theta}\right)=\log \left[f^{\prime} \circ\left(g_{j} \circ h\right)\left(y_{i}\right)\right]+\log \left[\left(g_{j}^{\prime} \circ h\right)\left(y_{i}\right)\right]+\log \left[-h^{\prime}\left(y_{i}\right)\right], \quad j=1,2 .
$$

Para $\lambda=\mu$, o logaritmo da função densidade fica

$$
\begin{aligned}
\log f_{T}\left(y_{i} ; \rho, \boldsymbol{\beta}, \boldsymbol{\theta}\right)= & \rho \frac{\gamma_{1}(t ; \boldsymbol{\beta}, \boldsymbol{\theta})}{\gamma_{1}\left(\xi_{i} ; \boldsymbol{\beta}, \boldsymbol{\theta}\right)}-\log \left(e^{\rho}-1\right)+\log \rho+ \\
& -\log \left\{\left[e^{\mathbf{x}_{i}^{\top} \boldsymbol{\beta}} \xi_{i}\left(1-S_{V}\left(y_{i} ; \boldsymbol{\theta}\right)\right)+1\right]^{2}\right\}+f_{V}\left(y_{i} ; \boldsymbol{\theta}\right) .
\end{aligned}
$$

No caso em que $\lambda \neq \mu$,

$$
\begin{aligned}
\log f_{T}\left(y_{i} ; \rho, \boldsymbol{\beta}, \boldsymbol{\theta}\right)= & \rho \frac{\gamma_{2}(t ; \boldsymbol{\beta}, \boldsymbol{\theta})}{\gamma_{2}\left(\xi_{i} ; \boldsymbol{\beta}, \boldsymbol{\theta}\right)}-\log \left(e^{\rho}-1\right)+\log \rho+\log \left[\left(e^{\mathbf{x}_{i}^{\top} \boldsymbol{\beta}}-e^{g\left(\mathbf{x}_{i}^{\top} \boldsymbol{\beta}\right)}\right)^{2}\right]+ \\
& +\left(e^{\mathbf{x}_{i}^{\top} \boldsymbol{\beta}}-e^{g\left(\mathbf{x}_{i}^{\top} \boldsymbol{\beta}\right)}\right) \xi_{i}-\log \left\{\left[e^{\mathbf{x}_{i}^{\top} \boldsymbol{\beta}} S_{V}\left(y_{i} ; \boldsymbol{\theta}\right)-e^{g\left(\mathbf{x}_{i}^{\top} \boldsymbol{\beta}\right)}+\right.\right. \\
& \left.\left.+e^{\mathbf{x}_{i}^{\top} \boldsymbol{\beta}}\left(1-S_{V}\left(y_{i} ; \boldsymbol{\theta}\right)\right) \exp \left\{\left(e^{\mathbf{x}_{i}^{\top} \boldsymbol{\beta}}-e^{g\left(\mathbf{x}_{i}^{\top} \boldsymbol{\beta}\right)}\right) \xi_{i}\right\}\right]^{2}\right\}+f_{V}\left(y_{i} ; \boldsymbol{\theta}\right) .
\end{aligned}
$$

Dessa forma, pode-se escrever a verossimilhança para o modelo proposto como

$$
\mathcal{L}_{n}(\boldsymbol{\beta}, \boldsymbol{\theta} ; \boldsymbol{y})=\mathcal{L}_{n, 1}(\boldsymbol{\beta}, \boldsymbol{\theta} ; \boldsymbol{y}) \mathbb{1}_{\{\lambda=\mu\}}+\mathcal{L}_{n, 2}(\boldsymbol{\beta}, \boldsymbol{\theta} ; \boldsymbol{y}) \mathbb{1}_{\{\lambda \neq \mu\}},
$$


com

$$
\begin{aligned}
\mathcal{L}_{n, 1}(\boldsymbol{\beta}, \boldsymbol{\theta} ; \boldsymbol{y})= & \prod_{i=1}^{n} \mathcal{L}_{1}\left(\boldsymbol{\beta}, \boldsymbol{\theta} ; y_{i}\right) \\
= & \prod_{i=1}^{n} f_{T}\left(y_{i} ; \boldsymbol{\beta}, \boldsymbol{\theta}\right)^{\delta_{i}} S_{T}\left(y_{i} ; \boldsymbol{\beta}, \boldsymbol{\theta}\right)^{\left(1-\delta_{i}\right)} \\
\log \mathcal{L}_{n, 1}(\boldsymbol{\beta}, \boldsymbol{\theta} ; \boldsymbol{y})= & \sum_{i=1}^{n}\left\{\delta_{i} \log f_{T}\left(y_{i} ; \boldsymbol{\beta}, \boldsymbol{\theta}\right)+\left(1-\delta_{i}\right) \log S_{T}\left(y_{i} ; \boldsymbol{\beta}, \boldsymbol{\theta}\right)\right\} \\
= & \sum_{i=1}^{n}\left\{\delta _ { i } \left[\rho \frac{\gamma_{1}(t ; \boldsymbol{\beta}, \boldsymbol{\theta})}{\gamma_{1}\left(\tau_{i}^{0} ; \boldsymbol{\beta}, \boldsymbol{\theta}\right)}-\log \left\{\left[e^{\mathbf{x}_{i}^{\top} \boldsymbol{\beta}} \tau_{i}^{0}\left(1-S_{V}\left(y_{i} ; \boldsymbol{\theta}\right)\right)+1\right]^{2}\right\}+\right.\right. \\
& \left.\left.+f_{V}\left(y_{i} ; \boldsymbol{\theta}\right)\right]+\left(1-\delta_{i}\right) \log \left(\exp \left\{\rho \frac{\gamma_{1}(t ; \boldsymbol{\beta}, \boldsymbol{\theta})}{\gamma_{1}\left(\tau_{i}^{0} ; \boldsymbol{\beta}, \boldsymbol{\theta}\right)}\right\}-1\right)\right\}+ \\
& +r \log \rho-n \log \left(e^{\rho}-1\right)
\end{aligned}
$$

e

$$
\begin{aligned}
\mathcal{L}_{n, 2}(\boldsymbol{\beta}, \boldsymbol{\theta} ; \boldsymbol{y})= & \prod_{i=1}^{n} \mathcal{L}_{2}\left(\boldsymbol{\beta}, \boldsymbol{\theta} ; y_{i}\right) \\
= & \prod_{i=1}^{n} f_{T}\left(y_{i} ; \boldsymbol{\beta}, \boldsymbol{\theta}\right)^{\delta_{i}} S_{T}\left(y_{i} ; \boldsymbol{\beta}, \boldsymbol{\theta}\right)^{\left(1-\delta_{i}\right)} \\
\log \mathcal{L}_{n, 2}(\boldsymbol{\beta}, \boldsymbol{\theta} ; \boldsymbol{y})= & \sum_{i=1}^{n}\left\{\delta_{i} \log f_{T}\left(y_{i} ; \boldsymbol{\beta}, \boldsymbol{\theta}\right)+\left(1-\delta_{i}\right) \log S_{T}\left(y_{i} ; \boldsymbol{\beta}, \boldsymbol{\theta}\right)\right\} \\
= & \sum_{i=1}^{n}\left\{\delta _ { i } \left[\rho \frac{\gamma_{2}(t ; \boldsymbol{\beta}, \boldsymbol{\theta})}{\gamma_{2}\left(\tau_{i}^{0} ; \boldsymbol{\beta}, \boldsymbol{\theta}\right)}-\log \left\{\left[e^{\mathbf{x}_{i}^{\top} \boldsymbol{\beta}} S_{V}\left(y_{i} ; \boldsymbol{\theta}\right)-e^{g\left(\mathbf{x}_{i}^{\top} \boldsymbol{\beta}\right)}+\right.\right.\right.\right. \\
& \left.\left.+e^{\mathbf{x}_{i}^{\top} \boldsymbol{\beta}}\left(1-S_{V}\left(y_{i} ; \boldsymbol{\theta}\right)\right) \exp \left\{\left(e^{\mathbf{x}_{i}^{\top} \boldsymbol{\beta}}-e^{g\left(\mathbf{x}_{i}^{\top} \boldsymbol{\beta}\right)}\right) \tau_{i}^{0}\right\}\right]^{2}\right\}+ \\
& \left.\left.+f_{V}\left(y_{i} ; \boldsymbol{\theta}\right)\right]+\left(1-\delta_{i}\right) \log \left(\exp \left\{\rho \frac{\gamma_{2}(t ; \boldsymbol{\beta}, \boldsymbol{\theta})}{\gamma_{2}\left(\tau_{i}^{0} ; \boldsymbol{\beta}, \boldsymbol{\theta}\right)}\right\}-1\right)\right\}+ \\
& +r \log \rho-n \log \left(e^{\rho}-1\right),
\end{aligned}
$$

em que $r=\sum_{i=1}^{n} \delta_{i}$

Assim como no modelo de tempos de promoção adaptado, a obtenção das estimativas de máxima verossimilhança fez uso do método iterativo de Newton-Raphson. Segundo esse procedimento, a partir de 
um valor inicial $\psi_{0}=\left(\boldsymbol{\beta}_{0}, \boldsymbol{\theta}_{0}\right)$, itera-se

$$
\left[\begin{array}{l}
\boldsymbol{\beta}_{k} \\
\boldsymbol{\theta}_{k}
\end{array}\right]=\left[\begin{array}{c}
\boldsymbol{\beta}_{k-1} \\
\boldsymbol{\theta}_{k-1}
\end{array}\right]+\left[\mathcal{I}_{F}\left(\boldsymbol{\psi}_{k-1}\right)\right]^{-1}\left[\begin{array}{l}
\mathcal{U}_{\boldsymbol{\beta}}\left(\boldsymbol{\psi}_{k-1}\right) \\
\mathcal{U}_{\boldsymbol{\theta}}\left(\boldsymbol{\psi}_{k-1}\right)
\end{array}\right]
$$

até que a distância euclidiana entre duas estimativas respectivas seja menor que uma constante positiva arbitrária. Como antes, $\mathcal{U}_{\beta}$ e $\mathcal{U}_{\boldsymbol{\theta}}$ são os vetores escore associados aos parâmetros $\boldsymbol{\beta}$ e $\boldsymbol{\theta}$ respectivamente e $\mathcal{I}_{F}$ é a informação de Fisher observada. 


\section{Capítulo 3}

\section{Estudos de simulação}

O objetivo dos procedimentos de simulação considerados são: avaliar a eficiência do processo de estimação adotado, comparar a metodologia proposta com aquela desenvolvida para o modelo de tempos de promoção e estudar o comportamento do modelo sob diferentes configurações dos parâmetros e tamanho de amostra.

O modelo em desenvolvimento foi avaliado utilizando-se dados simulados segundo diferentes configurações de tamanho de amostra e proporção de censura. A qualidade das estimativas obtidas foi avaliada com base na distância para os verdadeiros valores dos parâmetros bem como no desvio padrão das estimativas obtidas após as simulações. A comparação com o modelo de tempos de promoção se deu por meio do tempo mediano de sobrevivência, da fração de curados e do desvio máximo entre a função de sobrevivência estimada e a estimativa de Kaplan-Meier (Kaplan \& Meier, 1958).

As seções seguintes descrevem com detalhes as característica das amostras simuladas, o processo de simulação, o cálculo das quantidades utilizadas na seleção do modelo e avaliação da qualidade do ajuste.

\subsection{Geração dos dados}

Para a simulação de dados buscou-se a reprodução dos processos que motivaram o modelo proposto. Para evitar a necessidade de especificação de uma distribuição para os tempos de censura optou-se por considerar a ocorrência de censura Tipo I generalizada, tipicamente associada à não observação do evento devido ao término do período de acompanhamento em estudos cujos pacientes não apresentam o mesmo 
tempo de entrada. Além disso, esse é o mecanismo de censura predominante nos dados do ICESP, conforme o observado nas curva de Kaplan-Meier apresentada na Figura 3.1

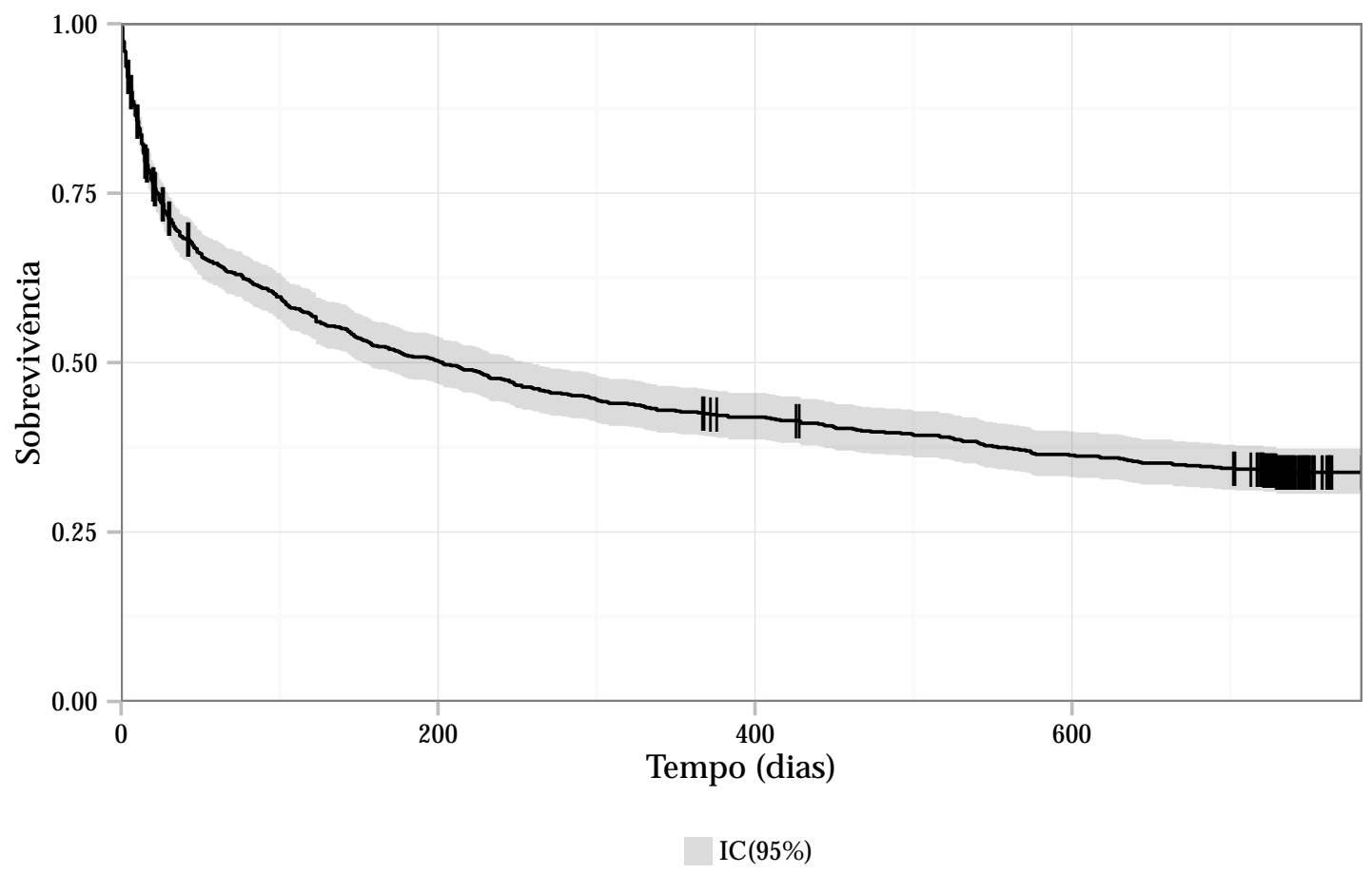

Figura 3.1: Curva de Kaplan-Meier para os 804 pacientes do ICESP.

De maneira geral, o procedimento para geração de dados considera a atribuição de um conjunto de valores para os parâmetros, a simulação do processo de nascimento e morte, a simulação dos tempo de sobrevivência e a atribuição de censuras de acordo com a proporção desejada. As principais etapas para a simulação de um tempo de acompanhamento são:

1. Atribuir valores à $\boldsymbol{\theta}, \boldsymbol{\beta}, v, \xi_{i}, w$ e inicializar um contador $i=1$;

2. $\operatorname{Gerar} \mathbf{x}_{i}^{\top}=\left[x_{i 1}, x_{i 2}, \ldots, x_{i p}\right]$;

3. Calcular $\lambda_{i}=e^{\mathbf{x}_{i}^{\top} \boldsymbol{\beta}}$ e $\mu_{i}=e^{g\left(\lambda_{i}\right)}$;

4. Declarar $y_{i}=0$ e, enquanto $y_{i}<\xi_{i}$,

4.1. Gerar o número inicial de células cancerígenas $m^{(0)}>0$ a partir de uma distribuição de Poisson com média $\rho$; 
4.2. Inicializar $r=1$, declarar $\zeta^{(0)}=0$ e atribuir $M^{(r)}=m^{(0)}$;

4.3. Enquanto $\sum_{k=1}^{r} \zeta^{(k)}<\xi_{i}$,

4.3.1. Gerar o tempo até a próxima transição dado por $\zeta^{(r+1)} \sim \operatorname{Exp}\left(M^{(r)}\left[\lambda_{i}+\mu_{i}\right]\right)$;

\subsubsection{Atualizar $r=r+1$;}

4.4. Gerar $z \sim \operatorname{Unif}(0,1)$ e, se $z<\mu_{i} /\left(\mu_{i}+\lambda_{i}\right)$, uma morte ocorre, i.e. $M^{(r+1)}=M^{(r)}-1$; caso contrário, um nascimento ocorre e $M^{(r+1)}=M^{(r)}+1$;

4.5. Caso $M^{(r)}=0$, atribuir $m^{\left(\xi_{i}\right)}=0$ e $y_{i}=+\infty$; caso contrário,

4.5.1. Gerar $u_{1}, \ldots, u_{m}\left(\xi_{i}\right)$ de $F_{U}(u ; \boldsymbol{\theta})$ e calcular $u=\min \left\{u_{1}, \ldots, u_{m}\left(\xi_{i}\right)\right\}$;

4.5.2. Atribuir $m^{\left(\xi_{i}\right)}=M^{(r)}$ e $y_{i}=(1-w) \xi_{i}+u$;

5. Atualizar $i=i+1$ e retornar ao passo 2 .

Após todos os tempo de sobrevivência terem sido gerados, aqueles designados como infinito (pertencentes aos indivíduos curados) são considerados censurados $\left(\delta_{i}=1\right)$ e têm seu valor truncado no tempo terminal

$$
\tau=y_{(n-r)}^{*}+2\left[y_{(n-r)}^{*}-y_{(n-r-1)}^{*}\right]
$$

em que $y_{(k)}^{*}$ é a $n$-ésima estatística de ordem entre os tempos de falha.

A fração de indivíduos curados naturalmente produz uma proporção $q$ de censura; porém, caso seja desejada uma proporção $p>q$ de tempos censurados, continua-se a atribuir aos maiores tempos de sobrevivência o tempo terminal até que a proporção de censurados desejada seja atingida.

\subsection{Simulação e estimação}

Nas amostras simuladas foram utilizadas distribuições Weibull para os tempos latentes, com função de sobrevivência dada por

$$
S_{U}\left(u ; \theta_{1}, \theta_{2}\right)=\exp \left\{-\left(\theta_{2} u\right)^{\theta_{1}}\right\}
$$

para $u \geq 0 \operatorname{com} \theta_{1}, \theta_{2}>0$. 
Foram mantidos fixos os valores $\theta_{1}=2$ e $\theta_{2}=0.005$, cuja análise dos dados do ICESP mostrou serem valores plausíveis. Mantiveram-se fixos, também, os valores de $\rho=5$ e $w=0$, representando respectivamente o número de fatores latentes no instante inicial e a constante conhecida denominada fator de remissão (ver p. 24).

A duração dos períodos de tratamento, $\xi_{i}$, foram simuladas segundo duas possíveis situações práticas: a primeira delas considera o mesmo período de tratamento para todos os pacientes, característica comum, por exemplo, em pacientes tratados com quimioterapia ou medicação específica, situações nas quais a duração do tratamento está predeterminada pelo protocolo médico; alternativamente pode-se pensar em diferentes períodos de tratamento, como, por exemplo, o acompanhamento feito durante o período de internação na UTI no caso dos pacientes do ICESP.

Aos períodos de tratamento fixos foram atribuídos $\xi_{i}=\xi=20$ dias. A simulação de tempos de tratamento individuais foi feita com base nos períodos de permanência na UTI observados nos pacientes do ICESP. Tendo esses tempos a distribuição apresentada no histograma da Figura 3.2 e um tempo mediano de 3 dias, optou-se por simular, para cada indivíduo, $\xi_{i}$ tempos com distribuição Exponencial de média 3.

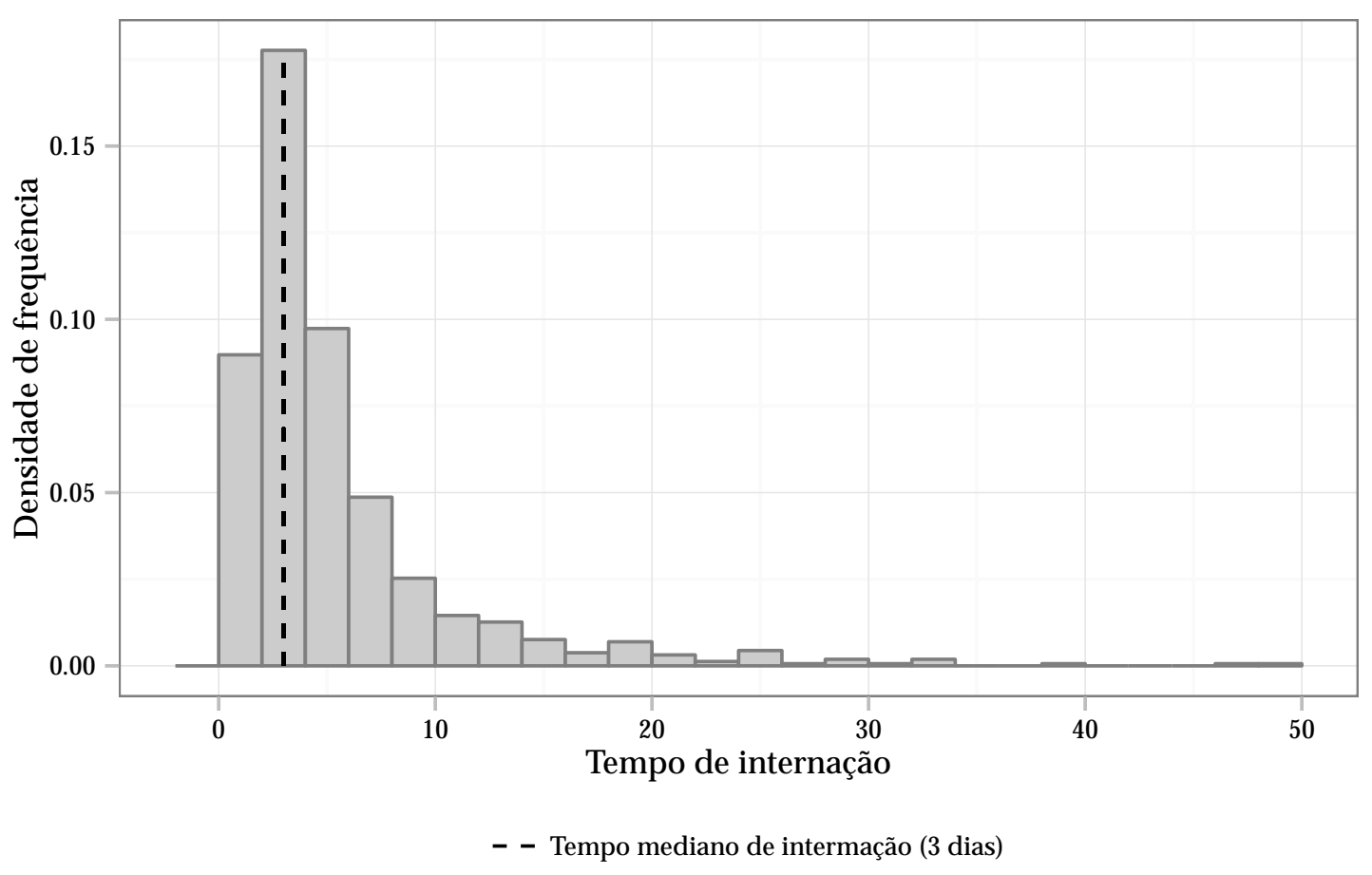

Figura 3.2: Histograma e tempo mediano para os tempos de permanência na UTI dos pacientes do ICESP. 


\begin{tabular}{ccc}
\hline & $\mu_{i}=\lambda_{i}$ & $\mu_{i} \neq \lambda_{i}$ \\
\hline$\xi=20$ & Configuração I & Configuração III \\
$\xi_{i} \sim \operatorname{Exp}(1 / 3)$ & Configuração II & Configuração IV \\
\hline
\end{tabular}

Tabela 3.1: Configurações das amostras geradas para simulação e análise do modelo proposto.

Para todas as amostras simuladas buscou-se uma proporção global de curados em torno de 5\%, as demais censuras foram induzidas propositalmente segundo o mecanismo explicado anteriormente. Dessa forma, uma amostra simulada com proporção de censura de $40 \%$, por exemplo, deve ser entendida como tendo aproximadamente 5\% de indivíduos curados e 35\% de indivíduos censurados pelo término do período de acompanhamento. A baixa proporção de cura adotada pode ser justificada com base nos dados do ICESP, os quais censuras devido à cura devem ser bastante raras por ser um conjunto de dados proveniente de pacientes em estado grave.

Primeiramente foram consideradas populações contendo dois grupos de pacientes, simulando a existência de dois tratamentos. Para garantir o balanceamento entre os grupos foram simuladas variáveis Bernoulli com probabilidade de sucesso 0.5, representando:

$$
X_{i}= \begin{cases}0, & \text { Tratamento A } \\ 1, & \text { Tratamento B }\end{cases}
$$

As amostras, por sua vez, podem ser classificadas em quatro configurações de acordo com as características apresentadas na Tabela 3.1. Cada configuração contém amostras de tamanho 500, 2000, 5000 e proporção de censura global de 20\%,60\% e mínima correspondendo apenas aos indivíduos curados (aproximadamente 5\%), totalizando 36 tipos diferentes de amostras a serem simuladas.

Para as amostras com configurações I e II foram utilizados como parâmetros de regressão o intercepto $\beta_{0}=-3$ e o efeito de tratamento $\beta_{1}=-1$, além de $v=1$ para que as taxas de nascimento e morte fossem iguais. Nas amostras com características III e IV atribuiu-se $\beta_{0}=-8$ e $\beta_{1}=2$.

Além disso, adotou-se

$$
g\left(\eta_{i}\right)=v \eta_{i}
$$

implicando na relação log-linear $\mu_{i}=\lambda_{i}^{v}$. Como o comportamento das estimativas dos parâmetros do 
modelo se mostrou semelhante para $\lambda_{i}<\mu_{i}$ e $\lambda_{i}>\mu_{i}$, optou-se por apenas um dos casos fazendo $v=0.5$

Para cada uma das 36 configurações foram criadas 2000 amostras para ajuste do modelo proposto e de tempos de promoção. As estimativas de máxima verossimilhança foram obtidas segundo o método descrito na Seção 2.4, utilizando a função maxLik (pacote maxLik versão 1.2-0 de Henningsen \& Toomet, 2011) disponível para o programa estatístico R (versão 3.0.2, R Core Team, 2012). Após a obtenção das estimativas, procedeu-se com a análise descritiva dos resultados por meio de gráficos do tipo boxplot e cálculo dos estimadores de Monte Carlo (Robert \& Casella, 2004) para média e desvio padrão das estimativas.

\subsection{Seleção de modelos}

Os critérios adotados para comparação do modelo proposto com o modelo de tempos de promoção foram: a distância entre a estimativa de máxima verossimilhança da curva de sobrevivência e a curva de Kaplan-Meier e as estimativas de máxima verossimilhança do tempo mediano e da proporção de cura.

As distâncias entre as estimativas de máxima verossimilhança da função de sobrevivência e as curvas de Kaplan-Meier foram definidas, para o modelo de tempos de promoção adaptado e proposto respectivamente, como

$$
d_{1}=\max _{0 \leq t \leq \tau}\left\{\left|\widehat{S}_{T}(t ; \widehat{\boldsymbol{\lambda}}, \widehat{\boldsymbol{\theta}})-\widehat{S}_{K M}(t)\right|\right\} \quad \text { e } \quad d_{2}=\max _{0 \leq t \leq \tau}\left\{\left|\widehat{S}_{T}(t ; \widehat{\rho}, \widehat{\lambda}, \widehat{\boldsymbol{\theta}})-\widehat{S}_{K M}(t)\right|\right\}
$$

em que $\widehat{S}_{T}(\cdot)$ é a estimativa de máxima verossimilhança da função de sobrevivência e $\widehat{S}_{K M}(\cdot)$ é a função escada definida pelo estimador Kaplan-Meier.

Como método para escolha entre diferentes funções que relacionam as taxas de nascimento e morte propõe-se o uso de critérios de informação e reamostragens do tipo bootstrap. Considerando o BIC como medida de informação, o qual penaliza a log-verossimilhança de acordo com o número de parâmetros presentes no modelo, tem-se que

$$
\mathrm{BIC}=-2 \mathscr{L}_{n}(\widehat{\boldsymbol{\lambda}}, \widehat{\boldsymbol{\theta}} ; \boldsymbol{y})+p \log n
$$

em que $p$ é o número de parâmetros do modelo e $n$ o tamanho da amostra.

Dada uma amostra e um conjunto de relações

$$
\mathscr{C}=\left\{g_{k}: k=1, \ldots, K\right\},
$$


as quais se deseja escolher a mais adequada aos dados, o método proposto consiste em simular $R$ amostras bootstrap a partir dos dados originais, ajustar o modelo e calcular o BIC sob cada uma das relações para cada amostra gerada. Ao final desse processo, avalia-se a seguinte quantidade

$$
c_{r, k}= \begin{cases}1, & \text { se }(\mathrm{BIC})_{r, k}=\min \left\{(\mathrm{BIC})_{r, h}: h=1, \ldots, K\right\} \\ 0, & \text { c.c. } ;\end{cases}
$$

em que (BIC) $)_{r, k}$ é o valor da medida de informação obtida no ajuste do modelo para a $r$-ésima amostra sob a $k$-ésima relação funcional entre as taxas de nascimento e morte. Calculam-se então as proporções

$$
p_{k}=\frac{1}{R} \sum_{r=1}^{R} c_{r, k}
$$

e seleciona-se a relação $\log \mu_{i}=g_{h}\left(\eta_{i}\right)$ em que $h$ é tal que

$$
p_{h}=\max \left\{p_{k}: k=1, \ldots, K\right\}
$$

Para verificar a eficiência do método de seleção de modelos proposto, adotou-se o procedimento descrito para amostras de cada um dos quatro grupos de configurações consideradas e verificou-se a relação funcional selecionada considerando um conjunto de valores pré-especificados para $v$ em (3.2).

\subsection{Resultados}

Os apêndices A e B contêm, respectivamente, as tabelas e os gráficos para análise descritiva das estimativas obtidas. Com relação à estimação dos parâmetros do modelo proposto, destacam-se as estimativas das quantidades latentes $\theta_{1}$ e $\theta_{2}$. Para esses parâmetros, foram obtidas estimativas consistentes sob todas configurações amostrais, proporções de censura e tamanho de amostra; as estimativas de Monte Carlo mais distantes dos verdadeiros valores utilizados foram observadas para $\theta_{2}$ sob $20 \%$ de censura, tamanhos amostrais 500 e 2000, e configurações II e IV.

A média da distribuição de Poisson utilizada na marginalização do modelo mostrou ser o parâmetro com estimativas mais instáveis. Fortemente influenciadas pelo aumento da proporção de censura, as 
estimativas desse parâmetro apresentaram alto viés e variabilidade quando considerada $60 \%$ de censura sob as configurações I e II.

As estimativas para os regressores $\beta_{0}$ e $\beta_{1}$ são, em geral, consistentes. Pode-se observar que a precisão e acurácia das estimativas desses parâmetros parecem ser influenciadas pelo tamanho amostral e proporção de censura. A utilização de tempos de tratamento adjuvante individuais acarretou uma maior variabilidade nas estimativas dessas quantidades.

As proporções de cura estimadas utilizando processos de nascimento e morte são, em geral, mais acuradas e consistentes que as estimativas obtidas segundo o modelo de tempos de promoção. Nota-se que as estimativas obtidas sob ambas metodologias são influências pelo nível de censura e tamanho amostral adotados, porém, o modelo proposto parece ser mais robusto, uma vez que apresentou maior precisão que o modelo usual nos casos em que a proporção de censuras é elevada.

O tempo mediano de sobrevivência foi melhor estimado utilizando-se modelo proposto, sendo as estimativas menos enviesadas sob todas as configurações e mais precisas sob as configurações I, II e IV. Com relação à aderência da curva de sobrevivência estimada à curva do estimador Kaplan-Meier, observa-se que o modelo de tempos de promoção produziu curvas mais aderentes e com menor variabilidade sob as configurações II e IV; nos demais casos o modelo proposto mostrou-se melhor, exceto quando considerada $60 \%$ de censura sob a configuração I.

Durante as simulações foi observado que, de maneira geral, o algoritmo de maximização da verossimilhança não obteve dificuldades de convergência, sendo necessárias pouco mais de cem iterações para a obtenção das estimativas finais para a maioria dos ajustes realizados. Porém, em alguns casos, as estimativas parecem ser influenciadas significativamente pelo valor inicial utilizado no processo de optimização. Esse problema parece afetar principalmente as estimativas nos casos em que $\lambda_{i} \neq \mu_{i}$, sendo o caso complementar robusto quanto à variação do ponto inicial. Sabendo da instabilidade apresentada pelo processo iterativo buscou-se utilizar valores iniciais não muito discrepantes dos verdadeiros valores dos parâmetros.

De maneira geral, pode-se considerar a metodologia consistente quanto à variação do tamanho de amostra e proporção de censura, pois observou-se a redução do viés e do erro padrão das estimativas com o aumento do tamanho da amostra e diminuição da proporção de censuras na maioria dos casos simulados.

Comparando o modelo proposto com o modelo de tempos de promoção, observa-se que a variabili- 
dade das estimativas das proporções de curados e tempos medianos obtidas considerando o processo de nascimento e morte na modelagem são, em geral, menores do que quando considera-se a modelagem usual. Muitas vezes esse comportamento se verifica mesmo nos casos de amostras com grande proporção de censuras e tamanho reduzido. A distância entre a estimativa de máxima verossimilhança e a curva de Kaplan-Meier tende a ser menor para o modelo proposto sob as configurações I e III. 



\section{Capítulo 4}

\section{Aplicação}

O banco de dados cedido pelo Instituto do Câncer do Estado de São Paulo (ICESP) contém 804 pacientes oncológicos acompanhados entre 2010 e 2012. Como parte do desenho amostral adotado, todos os pacientes foram encaminhados à Unidade de Tratamento Intensivo (UTI) durante algum período de seu acompanhamento médico. Nessa aplicação estudaremos o tempo de sobrevivência dos pacientes após o tratamento intensivo e tomaremos como origem o instante de entrada na UTI.

Apesar do grave quadro clínico apresentado por esses pacientes, notamos por meio da curva de KaplanMeier (Figura 3.1) que uma expressiva proporção (34.9\%) de indivíduos não apresenta o evento até o término do estudo. Dessa forma, a adoção de um modelo com fração de curados parece razoável; além disso, a curva de Kaplan-Meier revela uma concentração de censuras no período final de acompanhamento, o que pode contribuir para a adequabilidade do modelo proposto nesse trabalho aos dados.

O período de internação na UTI e o posterior acompanhamento médico podem ser identificados como as fases de progressão e promoção do modelo proposto. A primeira aplicação da nova metodologia a dados reais considerou dois grupos: o de pacientes que realizaram radioterapia durante algum período de seu tratamento (Tratamento A) e o grupo dos indivíduos cuja terapia de radiação não foi indicada (Tratamento B). Sabe-se que os pacientes submetidos a radioterapia, em geral, encontram-se em estado mais grave, o que pode ser confirmado a partir das curvas de Kaplan-Meier apresentadas na Figura 4.1

Adotou-se primeiramente a abordagem que considera o período de tratamento fixo; para tanto, tomou-se o tempo mediano de internação, 3 dias (ver Figura 3.2), como valor de referência para todos os pacientes. 


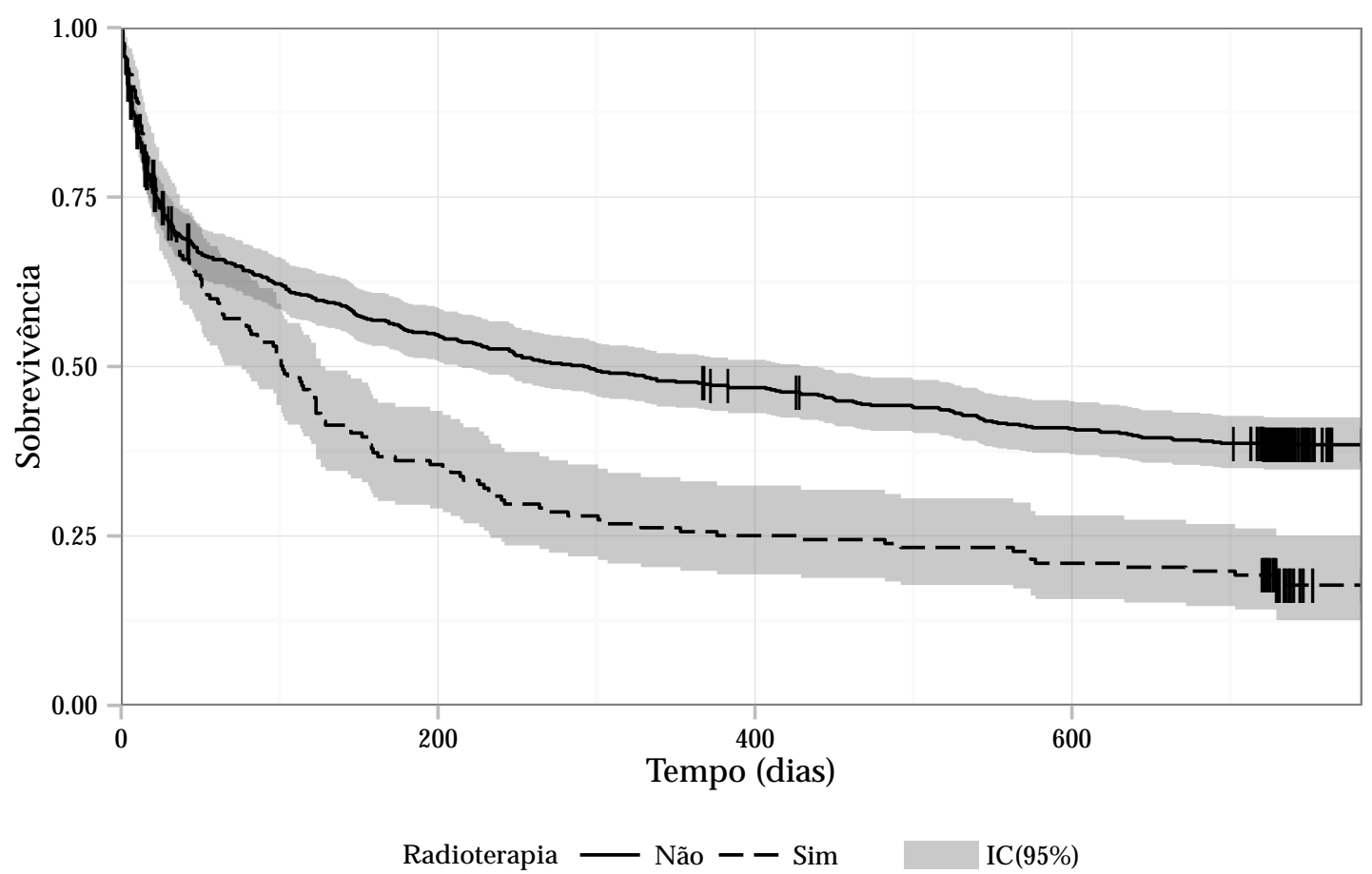

Figura 4.1: curvas de Kaplan-Meier para os pacientes do ICESP segundo a administração de radioterapia.

Além disso, foram considerados tempos latentes com distribuição Weibul de parâmetros $\boldsymbol{\theta}=\left(\theta_{1}, \theta_{2}\right)$, fator de remissão $w=0$, regressores

$$
\log \lambda_{i}=\beta_{0}+\beta_{1} x_{i} \quad \text { e } \quad \log \mu_{i}=v \log \lambda_{i}
$$

em que $x_{i}$ é 1 se o $i$-ésimo paciente fez radioterapia ( 0 caso contrário) e $v$ um valor conhecido préespecificado.

Para os 749 pacientes que sobreviveram aos 3 primeiros dias na UTI, a aplicação do método de seleção de modelos (Seção 3.3) a partir de um conjunto de relações $g_{k}\left(\eta_{i}\right)=v_{k} \eta_{i}$ e $R=5000$ amostras bootstrap resultou nas proporções observadas na Tabela 4.1. Nota-se que em 57 \% das reamostragens consideradas, a relação $\log \mu_{i}=2.5 \log \lambda_{i}$ foi selecionada como tendo o menor valor de BIC, logo, essa relação será a adotada na aplicação final do modelo.

Ajustando o modelo selecionado para os dados, obtêm-se as estimativas e erros padrões apresentados na Tabela 4.2. Como não foram verificadas as propriedades assintóticas dos estimadores de máxima 


\begin{tabular}{rrrrrrrrrr}
\hline$v_{k}$ & -1 & -0.5 & 0 & 0.5 & 1 & 1.5 & 2 & 2.5 & 3 \\
\hline$p_{k}$ & 0.0006 & 0.0004 & 0.0006 & 0.0006 & 0.1684 & 0.1584 & 0.0358 & 0.5724 & 0.0628 \\
\hline
\end{tabular}

Tabela 4.1: proporção de relações entre taxas selecionadas segundo o BIC para os dados do ICESP.

verossimilhança para o modelo proposto, calculou-se também estimativas para o desvio padrão baseadas em reamostragens jacknife (Quenouille, 1949; Shao \& Tu, 1995). As estimativas obtidas segundo os dois métodos foram próximas, o que evidencia a estabilidade do modelo ajustado aos dados do ICESP

\begin{tabular}{rrrr}
\hline Parâmetro & Estimativa & $\begin{array}{r}\text { Erro padrão } \\
\text { assintótico }\end{array}$ & $\begin{array}{r}\text { Erro padrão } \\
\text { jacknife }\end{array}$ \\
\hline $\boldsymbol{\theta}_{\mathbf{1}}$ & 0.7449 & 0.0432 & 0.0621 \\
$\boldsymbol{\theta}_{\mathbf{2}}$ & 0.0007 & 0.0003 & 0.0005 \\
$\boldsymbol{\beta}_{\mathbf{0}}$ & -0.2094 & 0.0618 & 0.0864 \\
$\boldsymbol{\beta}_{\mathbf{1}}$ & -0.6060 & 0.2328 & 0.2593 \\
$\boldsymbol{\rho}$ & 2.0857 & 0.6142 & 0.7541 \\
\hline
\end{tabular}

Tabela 4.2: estimativas e erros padrões para os parâmetros do modelo proposto ajustado aos dados do Instituto do Câncer.

Segundo as estimativas obtidas, a taxa de nascimento de clonogens no indivíduos que não foram submetidos à radioterapia é 83\% maior que a taxa de nascimento nos indivíduos tratados com radioterapia, pois

$$
e^{-\widehat{\beta}_{1}}=\frac{\widehat{\lambda}_{\mathrm{B}}}{\widehat{\lambda}_{\mathrm{A}}} \approx 1.83
$$

em que $\lambda_{\mathrm{A}}$ e $\lambda_{\mathrm{B}}$ são as taxas de nascimento para pacientes submetidos aos tratamentos A (com radioterapia) e B (sem radioterapia), respectivamente. Entretanto, a taxa de morte das células cancerígenas no grupo de pacientes não irradiados é, aproximadamente, 3.5 vezes maior que a taxa de morte no grupo de pacientes tratados com radioterapia, pois

$$
e^{-\widehat{\beta}_{1} v}=\frac{\widehat{\mu}_{\mathrm{B}}}{\widehat{\mu}_{\mathrm{A}}} \approx 4.55
$$

em que $\mu_{\mathrm{A}}$ e $\mu_{\mathrm{B}}$ são as taxas de morte para pacientes submetidos aos tratamentos A e $\mathrm{B}$, respectivamente

As estimativas de máxima verossimilhança e erros padrões jacknife para as proporções de cura, tempos medianos e distâncias máximas segundo o modelo proposto e o de tempos de promoção são apresentadas na Tabela 4.3. Apesar da metodologia proposta apresentar maior variabilidade nas estimativas, as distâncias máximas observadas em ambos os tratamentos foram menores quando considerado o processo de nascimento 
e morte.

\begin{tabular}{cccccccc}
\hline & \multicolumn{2}{c}{ Proporção. de cura } & \multicolumn{2}{c}{ Tempo mediano } & \multicolumn{2}{c}{ Distância máxima } \\
\cline { 2 - 7 } Tratamento & A & B & A & B & A & B \\
\hline Modelo proposto & $0.320(0.058)$ & $0.075(0.066)$ & $313(53)$ & $113(22)$ & $0.0504(0.0108)$ & $0.0440(0.0246)$ \\
Tempos de promoção & $0.335(0.031)$ & $0.150(0.031)$ & $329(49)$ & $102(16)$ & $0.0669(0.0097)$ & $0.0638(0.0176)$ \\
\hline
\end{tabular}

Tabela 4.3: estimativas e erros padrões da proporção de curados, tempo mediano e distância máxima para os tratamentos A e B segundo o modelo de tempos de promoção adaptado e o modelo proposto considerando os dados do ICESP.

O ajuste da curva de sobrevivência estimada para ambos os modelos pode ser observado na Figura 4.2. Para o grupo de indivíduos tratados com radioterapia, notou-se um melhor ajuste da curva de sobrevivência estimada durante todo o período de acompanhamento. Para os demais pacientes, nota-se que a curva estimada apresentou melhor ajuste nos períodos inicial e final do estudo.

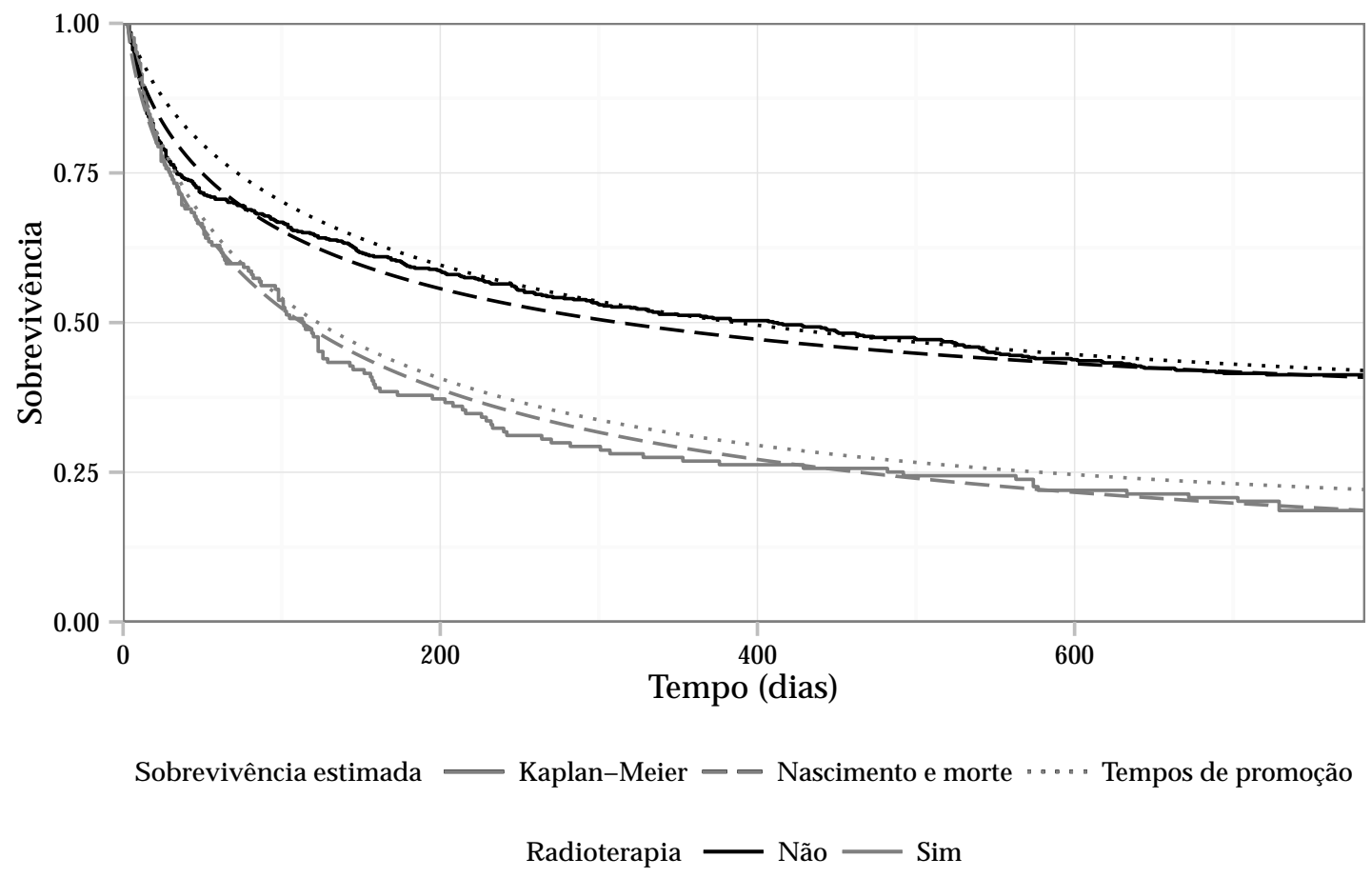

Figura 4.2: curvas de Kaplan-Meier e funções de sobrevivência estimadas segundo o modelo proposto e o de tempos de promoção para os dados do ICESP. 


\section{Capítulo 5}

\section{Discussões e pesquisas futuras}

A mensuração da utilidade da metodologia proposta e de seu potencial como técnica alternativa para análise de dados de longa duração depende ainda de uma vasta investigação. Pesquisas em andamento envolvem a variação de algumas das suposições feitas durante a formulação do modelo e simulação dos dados. Em seguida deverão ser consideradas algumas formulações alternativas e desenvolvimento formal da teoria assintótica para o modelo proposto. Encontra-se ainda em estágio inicial de desenvolvimento uma abordagem Bayesiana para a modelagem proposta.

Durante a formulação do modelo adotou-se um valor estritamente positivo para número inicial de fatores latentes, porém, é possível que essa não seja uma suposição razoável para algumas das aplicações. Assim, modificações na função de sobrevivência serão necessárias em virtude da utilização da distribuição de Poisson usual (não truncada). Com relação aos estudos de simulação, deseja-se avaliar o comportamento do modelo mediante variação no valor do fator de remissão e aumento da proporção de indivíduos efetivamente curados (indivíduos que não apresentaram evento devido à cura e não em virtude ao término precoce do estudo, ver a discussão detalhada na p. 39).

A adoção de diferentes modelos probabilísticos para os tempos latentes também deve ser considerada, bem como a utilização de diferentes funções de ligação na modelagem das taxas de nascimento e morte. Além disso, pretende-se avaliar a aplicação do modelo em dados simulados que violem as suposições impostas durante sua formulação, permitindo, assim, a avaliação da robustez da nova metodologia.

Diversas variações na formulação do modelo foram consideradas para futura investigação. Entre as mais 
relevantes estão a modelagem da razão entre as taxas de nascimento e morte, com o intuito de contornar a instabilidade observada quando modeladas as duas taxas separadamente. Idealizou-se também a ocorrência concomitante das fases de progressão e promoção, essa abordagem permitiria a utilização de dados colhidos durante o período de tratamento adjuvante e a avaliação da sobrevida dos pacientes em qualquer instante do estudo. Pretende-se considerar também as adaptações necessárias para a inclusão de variáveis dependente do tempo.

As derivações teóricas são necessárias para a adoção dos resultados assintóticos usuais envolvendo estimação de máxima verossimilhança.

A abordagem Bayesiana mostra-se promissora, pois novos aspectos poderiam ser considerados na formulação do modelo. A atribuição de uma distribuição a priori para o valor inicial do número de fatores latentes, por exemplo, eliminaria a necessidade da marginalização da função de sobrevivência. 


\section{Apêndice A}

\section{Tabelas}

A tabela seguinte tem como objetivo auxiliar a análise dos resultados obtidos nos estudos de simulação. Nela são listados o número e página de cada tabela contendo as estimativas de Monte Carlo para os parâmetros dos modelos trabalhados (modelo proposto de sobrevivência com fração de curados utilizando processo de nascimento e morte e o modelo de tempos de promoção) e para as estatísticas de interesse (proporção de curados, tempo mediano de sobrevivência e distância máxima entre as sobrevivências estimadas) utilizadas na análise e comparação dos modelos. Como foram simulados dois grupos de pacientes, os resultados aparecem ainda divididos segundo o tratamento considerado.

\begin{tabular}{|c|c|c|c|c|c|}
\hline Configuração & Estimativa & Tratamento & Modelo & Tabela & Página \\
\hline \multirow{6}{*}{ I } & \multirow{2}{*}{ parâmetros } & \multirow{2}{*}{-} & nascimento e morte & A. 1 & 51 \\
\hline & & & tempos de promoção & A. 2 & 51 \\
\hline & \multirow{4}{*}{ estatisticas } & \multirow{2}{*}{ A } & nascimento e morte & A. 3 & 51 \\
\hline & & & tempos de promoção & A. 4 & 52 \\
\hline & & \multirow{2}{*}{ B } & nascimento e morte & A. 5 & 52 \\
\hline & & & tempos de promoção & A. 6 & 52 \\
\hline \multirow{6}{*}{ II } & \multirow{2}{*}{ parâmetros } & \multirow{2}{*}{-} & nascimento e morte & A.7 & 53 \\
\hline & & & tempos de promoção & A. 8 & 53 \\
\hline & \multirow{4}{*}{ estatisticas } & \multirow{2}{*}{ A } & nascimento e morte & A.9 & 53 \\
\hline & & & tempos de promoção & A. 10 & 54 \\
\hline & & \multirow{2}{*}{ B } & nascimento e morte & A.11 & 54 \\
\hline & & & tempos de promoção & A. 12 & 54 \\
\hline
\end{tabular}




\begin{tabular}{|c|c|c|c|c|c|}
\hline Configuração & Estimativa & Tratamento & Modelo & Tabela & Página \\
\hline \multirow{6}{*}{ III } & \multirow{2}{*}{ parâmetros } & \multirow{2}{*}{-} & nascimento e morte & A.13 & 55 \\
\hline & & & tempos de promoção & A.14 & 55 \\
\hline & \multirow{4}{*}{ estatisticas } & \multirow{2}{*}{ A } & nascimento e morte & A.15 & 55 \\
\hline & & & tempos de promoção & A. 16 & 56 \\
\hline & & \multirow{2}{*}{$\mathrm{B}$} & nascimento e morte & A.17 & 56 \\
\hline & & & tempos de promoção & A.18 & 56 \\
\hline \multirow{6}{*}{ IV } & \multirow{2}{*}{ parâmetros } & \multirow{2}{*}{-} & nascimento e morte & A.19 & 57 \\
\hline & & & tempos de promoção & A. 20 & 57 \\
\hline & \multirow{4}{*}{ estatisticas } & \multirow{2}{*}{ A } & nascimento e morte & A. 21 & 57 \\
\hline & & & tempos de promoção & A. 22 & 58 \\
\hline & & \multirow{2}{*}{ B } & nascimento e morte & A.23 & 58 \\
\hline & & & tempos de promoção & A. 24 & 58 \\
\hline
\end{tabular}




\begin{tabular}{lllllll}
\hline Censura & $\boldsymbol{n}$ & $\boldsymbol{\theta}_{\mathbf{1}}$ & $\boldsymbol{\theta}_{\mathbf{2}}$ & $\boldsymbol{\beta}_{\mathbf{0}}$ & $\boldsymbol{\beta}_{\mathbf{1}}$ & $\boldsymbol{\rho}$ \\
\hline \multicolumn{2}{l}{ Verdadeiro valor } & 2 & 0.005 & -3 & -1 & 5 \\
\hline$\approx \mathbf{5 \%}$ & $\mathbf{5 0 0}$ & $2.0123(0.1066)$ & $0.0044(0.0011)$ & $-2.5227(0.9101)$ & $-1.1293(1.1123)$ & $8.9209(6.4882)$ \\
& $\mathbf{2 0 0 0}$ & $2.0068(0.0523)$ & $0.0048(0.0005)$ & $-2.8313(0.4311)$ & $-0.9660(0.2613)$ & $5.8434(1.7298)$ \\
& $\mathbf{5 0 0 0}$ & $2.0023(0.0320)$ & $0.0049(0.0003)$ & $-2.9283(0.2569)$ & $-0.9848(0.1630)$ & $5.3117(0.8352)$ \\
\hline $\mathbf{2 0} \%$ & $\mathbf{5 0 0}$ & $1.9881(0.1313)$ & $0.0082(0.0022)$ & $-4.0653(0.9261)$ & $-1.7157(1.9883)$ & $2.1355(2.7006)$ \\
& $\mathbf{2 0 0 0}$ & $1.9988(0.0714)$ & $0.0067(0.0018)$ & $-3.7048(0.8162)$ & $-1.4979(1.3094)$ & $3.0597(1.7497)$ \\
& $\mathbf{5 0 0 0}$ & $2.0021(0.0438)$ & $0.0051(0.0006)$ & $-3.0460(0.2597)$ & $-1.0670(0.4018)$ & $4.8952(0.7631)$ \\
\hline \multirow{6}{*00\%}{} & $\mathbf{5 0 0}$ & $2.1017(0.2134)$ & $0.0054(0.0016)$ & $-3.2871(2.0309)$ & $-1.1343(2.9471)$ & $6.7570(5.1621)$ \\
& $\mathbf{2 0 0 0}$ & $2.0302(0.1038)$ & $0.0046(0.0006)$ & $-2.9393(1.4372)$ & $-1.6727(2.5129)$ & $6.7834(2.3054)$ \\
& $\mathbf{5 0 0 0}$ & $1.9757(0.0762)$ & $0.0042(0.0008)$ & $-3.6037(1.3437)$ & $-2.6783(1.9856)$ & $7.0734(1.6340)$ \\
\hline
\end{tabular}

Tabela A.1: estimativas e erros padrões dos parâmetros do modelo proposto.

\begin{tabular}{llllll}
\hline Censura & $\boldsymbol{n}$ & $\boldsymbol{\theta}_{\mathbf{1}}$ & $\boldsymbol{\theta}_{\mathbf{2}}$ & $\boldsymbol{\beta}_{\mathbf{0}}$ & $\boldsymbol{\beta}_{\mathbf{1}}$ \\
\hline$\approx \mathbf{5 \%}$ & $\mathbf{5 0 0}$ & $1.8694(0.0672)$ & $0.0057(0.0003)$ & $1.0506(0.0883)$ & $0.2225(0.0882)$ \\
& $\mathbf{2 0 0 0}$ & $1.8765(0.0377)$ & $0.0058(0.0002)$ & $1.0353(0.0544)$ & $0.2199(0.0452)$ \\
& $\mathbf{5 0 0 0}$ & $1.8787(0.0215)$ & $0.0058(0.0001)$ & $1.0313(0.0264)$ & $0.2210(0.0293)$ \\
\hline $\mathbf{2 0 \%}$ & $\mathbf{5 0 0}$ & $1.9956(0.1209)$ & $0.0069(0.0012)$ & $0.8936(0.2944)$ & $0.1420(0.1024)$ \\
& $\mathbf{2 0 0 0}$ & $1.9967(0.0614)$ & $0.0070(0.0005)$ & $0.8489(0.1061)$ & $0.1445(0.0468)$ \\
& $\mathbf{5 0 0 0}$ & $1.9936(0.0378)$ & $0.0070(0.0003)$ & $0.8456(0.0621)$ & $0.1440(0.0307)$ \\
\hline $\mathbf{6 0 \%}$ & $\mathbf{5 0 0}$ & $2.0755(0.2105)$ & $0.0082(0.0046)$ & $1.1081(1.4201)$ & $0.0570(0.1385)$ \\
& $\mathbf{2 0 0 0}$ & $2.0201(0.1074)$ & $0.0075(0.0031)$ & $0.9904(0.8920)$ & $0.0561(0.0690)$ \\
& $\mathbf{5 0 0 0}$ & $2.0145(0.0775)$ & $0.0076(0.0022)$ & $0.8480(0.5900)$ & $0.0571(0.0436)$ \\
\hline
\end{tabular}

Tabela A.2: estimativas e erros padrões dos parâmetros do modelo de tempos de promoção.

\begin{tabular}{lllll}
\hline Censura & $\boldsymbol{n}$ & Prop. de cura & Tempo mediano & \multicolumn{1}{l}{$\boldsymbol{d}_{\max }$} \\
\hline \multicolumn{2}{l}{ Verdadeiro valor } & 0.0754 & 103.2331 & - \\
\hline$\approx \mathbf{5 \%}$ & $\mathbf{5 0 0}$ & $0.0708(0.0177)$ & $103.2189(2.9416)$ & $0.0441(0.0125)$ \\
& $\mathbf{2 0 0 0}$ & $0.0742(0.0083)$ & $103.2561(1.5002)$ & $0.0229(0.0064)$ \\
& $\mathbf{5 0 0 0}$ & $0.0749(0.0053)$ & $103.2035(0.9206)$ & $0.0146(0.0040)$ \\
\hline $\mathbf{2 0} \%$ & $\mathbf{5 0 0}$ & $0.1195(0.0370)$ & $102.8714(3.3243)$ & $0.0414(0.0125)$ \\
& $\mathbf{2 0 0 0}$ & $0.0995(0.0307)$ & $103.1204(1.5900)$ & $0.0213(0.0060)$ \\
& $\mathbf{5 0 0 0}$ & $0.0772(0.0137)$ & $103.2171(1.0741)$ & $0.0137(0.0038)$ \\
\hline $\mathbf{6 0} \%$ & $\mathbf{5 0 0}$ & $0.1555(0.1273)$ & $106.0291(8.1245)$ & $0.0314(0.0104)$ \\
& $\mathbf{2 0 0 0}$ & $0.0956(0.0757)$ & $103.7528(3.4618)$ & $0.0165(0.0051)$ \\
& $\mathbf{5 0 0 0}$ & $0.0519(0.0619)$ & $102.5500(2.5043)$ & $0.0113(0.0037)$ \\
\hline
\end{tabular}

Tabela A.3: estimativas e erros padrões das quantidades de interesse segundo o modelo proposto para os pacientes submetidos ao tratamento A. 


\begin{tabular}{lllll}
\hline Censura & $\boldsymbol{n}$ & Prop. de cura & Tempo mediano & \multicolumn{1}{l}{$\boldsymbol{d}_{\max }$} \\
\hline \multicolumn{2}{l}{ Verdadeiro valor } & 0.0754 & 103.2331 & - \\
\hline$\approx \mathbf{5 \%}$ & $\mathbf{5 0 0}$ & $0.0585(0.0142)$ & $107.9372(4.0197)$ & $0.0563(0.0141)$ \\
& $\mathbf{2 0 0 0}$ & $0.0602(0.0073)$ & $107.8274(2.0603)$ & $0.0411(0.0078)$ \\
& $\mathbf{5 0 0 0}$ & $0.0606(0.0045)$ & $107.7555(1.3238)$ & $0.0359(0.0052)$ \\
\hline $\mathbf{2 0 \%}$ & $\mathbf{5 0 0}$ & $0.0969(0.0407)$ & $104.4710(4.3377)$ & $0.0407(0.0114)$ \\
& $\mathbf{2 0 0 0}$ & $0.0983(0.0222)$ & $104.5686(1.9750)$ & $0.0250(0.0064)$ \\
& $\mathbf{5 0 0 0}$ & $0.0979(0.0138)$ & $104.5568(1.3058)$ & $0.0198(0.0045)$ \\
\hline \multirow{6}{*0\%}{} & $\mathbf{5 0 0}$ & $0.1957(0.1741)$ & $105.3887(8.0791)$ & $0.0272(0.0077)$ \\
& $\mathbf{2 0 0 0}$ & $0.1441(0.1241)$ & $103.3108(3.4026)$ & $0.0145(0.0039)$ \\
& $\mathbf{5 0 0 0}$ & $0.1370(0.0976)$ & $103.0774(2.2552)$ & $0.0097(0.0026)$ \\
\hline
\end{tabular}

Tabela A.4: estimativas e erros padrões das quantidades de interesse segundo o modelo de tempos de promoção para os pacientes submetidos ao tratamento A.

\begin{tabular}{lllll}
\hline Censura & $\boldsymbol{n}$ & Prop. de cura & Tempo mediano & $\boldsymbol{d}_{\max }$ \\
\hline \multicolumn{2}{l}{ Verdadeiro valor } & 0.0191 & 99.0278 & - \\
\hline$\approx \mathbf{5 \%}$ & $\mathbf{5 0 0}$ & $0.0156(0.0082)$ & $98.8777(2.6317)$ & $0.0438(0.0123)$ \\
& $\mathbf{2 0 0 0}$ & $0.0183(0.0041)$ & $98.9978(1.3595)$ & $0.0224(0.0061)$ \\
& $\mathbf{5 0 0 0}$ & $0.0187(0.0026)$ & $98.9667(0.8298)$ & $0.0145(0.0038)$ \\
\hline $\mathbf{2 0 \%}$ & $\mathbf{5 0 0}$ & $0.0501(0.0421)$ & $99.2683(2.8152)$ & $0.0422(0.0123)$ \\
& $\mathbf{2 0 0 0}$ & $0.0325(0.0252)$ & $99.2335(1.4751)$ & $0.0216(0.0063)$ \\
& $\mathbf{5 0 0 0}$ & $0.0210(0.0102)$ & $99.0589(0.8798)$ & $0.0137(0.0037)$ \\
\hline $\mathbf{6 0 \%}$ & $\mathbf{5 0 0}$ & $0.1034(0.1148)$ & $101.0693(6.2549)$ & $0.0311(0.0099)$ \\
& $\mathbf{2 0 0 0}$ & $0.0502(0.0611)$ & $99.7145(2.7484)$ & $0.0164(0.0050)$ \\
& $\mathbf{5 0 0 0}$ & $0.0195(0.0401)$ & $99.2508(1.6484)$ & $0.0115(0.0038)$ \\
\hline
\end{tabular}

Tabela A.5: estimativas e erros padrões das quantidades de interesse segundo o modelo proposto para os pacientes submetidos ao tratamento B.

\begin{tabular}{lllll}
\hline Censura & $\boldsymbol{n}$ & Prop. de cura & Tempo mediano & \multicolumn{1}{l}{$\boldsymbol{d}_{\max }$} \\
\hline \multicolumn{2}{l}{ Verdadeiro valor } & 0.0191 & 99.0278 & - \\
\hline$\approx \mathbf{5 \%}$ & $\mathbf{5 0 0}$ & $0.0288(0.0076)$ & $96.7964(3.0224)$ & $0.0479(0.0129)$ \\
& $\mathbf{2 0 0 0}$ & $0.0302(0.0040)$ & $96.8688(1.5509)$ & $0.0336(0.0076)$ \\
& $\mathbf{5 0 0 0}$ & $0.0303(0.0025)$ & $96.7658(0.9405)$ & $0.0288(0.0051)$ \\
\hline \multirow{20}{\mathbf{20}\%}{} & $\mathbf{5 0 0}$ & $0.0692(0.0332)$ & $97.6096(3.2315)$ & $0.0394(0.0107)$ \\
& $\mathbf{2 0 0 0}$ & $0.0689(0.0179)$ & $97.6224(1.6364)$ & $0.0245(0.0066)$ \\
& $\mathbf{5 0 0 0}$ & $0.0685(0.0113)$ & $97.6302(1.0137)$ & $0.0195(0.0045)$ \\
\hline \multirow{6}{*0\%}{} & $\mathbf{5 0 0}$ & $0.1827(0.1665)$ & $101.9178(6.7169)$ & $0.0268(0.0076)$ \\
& $\mathbf{2 0 0 0}$ & $0.1325(0.1174)$ & $100.4071(3.0902)$ & $0.0147(0.0040)$ \\
& $\mathbf{5 0 0 0}$ & $0.1242(0.0917)$ & $100.1592(1.8773)$ & $0.0098(0.0028)$ \\
\hline
\end{tabular}

Tabela A.6: estimativas e erros padrões das quantidades de interesse segundo o modelo de tempos de promoção para os pacientes submetidos ao tratamento B. 


\begin{tabular}{lllllll}
\hline Censura & $\boldsymbol{n}$ & $\boldsymbol{\theta}_{\mathbf{1}}$ & $\boldsymbol{\theta}_{\mathbf{2}}$ & $\boldsymbol{\beta}_{\mathbf{0}}$ & $\boldsymbol{\beta}_{\mathbf{1}}$ & $\boldsymbol{\rho}$ \\
\hline \multicolumn{2}{l}{ Verdadeiro valor } & 2 & 0.005 & -3 & -1 & 5 \\
\hline$\approx \mathbf{5 \%}$ & $\mathbf{5 0 0}$ & $1.9783(0.0795)$ & $0.0052(0.0013)$ & $-4.5105(3.0785)$ & $-2.8397(3.3058)$ & $5.3147(2.8968)$ \\
& $\mathbf{2 0 0 0}$ & $1.9858(0.0409)$ & $0.0049(0.0006)$ & $-3.0491(0.7327)$ & $-1.8780(2.3554)$ & $5.3536(1.2405)$ \\
& $\mathbf{5 0 0 0}$ & $1.9906(0.0260)$ & $0.0049(0.0004)$ & $-2.9526(0.3766)$ & $-1.1597(0.7770)$ & $5.2861(0.7100)$ \\
\hline $\mathbf{2 0} \%$ & $\mathbf{5 0 0}$ & $2.0044(0.1158)$ & $0.0085(0.0017)$ & $-4.7233(2.1163)$ & $-1.2286(2.9316)$ & $1.5308(1.3232)$ \\
& $\mathbf{2 0 0 0}$ & $2.0119(0.0647)$ & $0.0082(0.0013)$ & $-4.5000(1.6338)$ & $-3.0773(2.9911)$ & $1.6248(1.0507)$ \\
& $\mathbf{5 0 0 0}$ & $2.0079(0.0398)$ & $0.0052(0.0008)$ & $-3.2361(0.9394)$ & $-1.5913(1.6668)$ & $4.8854(1.5752)$ \\
\hline \multirow{6}{*00\%}{} & $\mathbf{5 0 0}$ & $2.0369(0.1415)$ & $0.0061(0.0018)$ & $-3.6549(2.7300)$ & $-1.5574(2.6980)$ & $5.2992(5.6721)$ \\
& $\mathbf{2 0 0 0}$ & $2.0199(0.0720)$ & $0.0057(0.0012)$ & $-3.8189(2.3294)$ & $-1.6873(2.2158)$ & $4.7366(3.3466)$ \\
& $\mathbf{5 0 0 0}$ & $1.9840(0.0442)$ & $0.0040(0.0004)$ & $-5.6834(2.2303)$ & $-2.3943(0.8722)$ & $7.4738(0.9422)$ \\
\hline
\end{tabular}

Tabela A.7: estimativas e erros padrões dos parâmetros do modelo proposto.

\begin{tabular}{llllll}
\hline Censura & $\boldsymbol{n}$ & $\boldsymbol{\theta}_{\mathbf{1}}$ & $\boldsymbol{\theta}_{\mathbf{2}}$ & $\boldsymbol{\beta}_{\mathbf{0}}$ & $\boldsymbol{\beta}_{\mathbf{1}}$ \\
\hline $\mathbf{5} \mathbf{5 \%}$ & $\mathbf{5 0 0}$ & $1.9343(0.0760)$ & $0.0044(0.0005)$ & $1.7589(0.1910)$ & $0.0469(0.0914)$ \\
& $\mathbf{2 0 0 0}$ & $1.9466(0.0392)$ & $0.0046(0.0002)$ & $1.6876(0.1395)$ & $0.0418(0.0450)$ \\
& $\mathbf{5 0 0 0}$ & $1.9525(0.0241)$ & $0.0046(0.0001)$ & $1.6665(0.0429)$ & $0.0429(0.0282)$ \\
\hline $\mathbf{2 0 \%}$ & $\mathbf{5 0 0}$ & $2.0147(0.1337)$ & $0.0051(0.0020)$ & $1.7784(0.9338)$ & $0.0198(0.0977)$ \\
& $\mathbf{2 0 0 0}$ & $2.0019(0.0725)$ & $0.0051(0.0011)$ & $1.5955(0.4204)$ & $0.0237(0.0504)$ \\
& $\mathbf{5 0 0 0}$ & $1.9999(0.0467)$ & $0.0052(0.0007)$ & $1.5380(0.2234)$ & $0.0219(0.0310)$ \\
\hline $\mathbf{6 0 \%}$ & $\mathbf{5 0 0}$ & $2.1421(2.7911)$ & $0.0068(0.0045)$ & $1.8636(10.2536)$ & $-0.0760(3.5440)$ \\
& $\mathbf{2 0 0 0}$ & $2.0348(0.1018)$ & $0.0060(0.0031)$ & $1.6021(1.1102)$ & $0.0081(0.0687)$ \\
& $\mathbf{5 0 0 0}$ & $2.0219(0.0652)$ & $0.0058(0.0023)$ & $1.5046(0.7967)$ & $0.0079(0.0444)$ \\
\hline
\end{tabular}

Tabela A.8: estimativas e erros padrões dos parâmetros do modelo de tempos de promoção.

\begin{tabular}{lllll}
\hline Censura & $\boldsymbol{n}$ & Prop. de cura & Tempo mediano & $\boldsymbol{d}_{\max }$ \\
\hline \multicolumn{2}{l}{ Verdadeiro valor } & 0.0062 & 80.7199 & - \\
\hline$\approx \mathbf{5 \%}$ & $\mathbf{5 0 0}$ & $0.0047(0.0042)$ & $80.9156(2.2952)$ & $0.0452(0.0127)$ \\
& $\mathbf{2 0 0 0}$ & $0.0053(0.0023)$ & $80.8099(1.1770)$ & $0.0233(0.0061)$ \\
& $\mathbf{5 0 0 0}$ & $0.0056(0.0014)$ & $80.7943(0.7582)$ & $0.0151(0.0040)$ \\
\hline $\mathbf{2 0 \%}$ & $\mathbf{5 0 0}$ & $0.0298(0.0275)$ & $80.9139(2.4791)$ & $0.0431(0.0126)$ \\
& $\mathbf{2 0 0 0}$ & $0.0235(0.0166)$ & $80.8380(1.2346)$ & $0.0222(0.0062)$ \\
& $\mathbf{5 0 0 0}$ & $0.0078(0.0055)$ & $80.6844(0.7944)$ & $0.0141(0.0039)$ \\
\hline \multirow{6}{*00\%}{} & $\mathbf{5 0 0}$ & $0.0544(0.0753)$ & $82.5735(4.7410)$ & $0.0346(0.0115)$ \\
& $\mathbf{2 0 0 0}$ & $0.0264(0.0357)$ & $81.3282(2.1562)$ & $0.0177(0.0057)$ \\
& $\mathbf{5 0 0 0}$ & $0.0043(0.0129)$ & $80.5293(1.3399)$ & $0.0119(0.0038)$ \\
\hline
\end{tabular}

Tabela A.9: estimativas e erros padrões das quantidades de interesse segundo o modelo proposto para os pacientes submetidos ao tratamento A. 


\begin{tabular}{lllll}
\hline Censura & $\boldsymbol{n}$ & Prop. de cura & Tempo mediano & $\boldsymbol{d}_{\max }$ \\
\hline \multicolumn{2}{l}{ Verdadeiro valor } & 0.0062 & 80.7199 & - \\
\hline$\approx \mathbf{5 \%}$ & $\mathbf{5 0 0}$ & $0.0042(0.0031)$ & $82.0093(2.9709)$ & $0.0431(0.0112)$ \\
& $\mathbf{2 0 0 0}$ & $0.0048(0.0018)$ & $81.6966(1.5422)$ & $0.0236(0.0060)$ \\
& $\mathbf{5 0 0 0}$ & $0.0051(0.0011)$ & $81.6905(0.9777)$ & $0.0173(0.0042)$ \\
\hline \multirow{2}{*}{$\mathbf{2 0} \%$} & $\mathbf{5 0 0}$ & $0.0245(0.0281)$ & $80.9416(3.2506)$ & $0.0371(0.0097)$ \\
& $\mathbf{2 0 0 0}$ & $0.0156(0.0155)$ & $80.9630(1.6421)$ & $0.0194(0.0049)$ \\
& $\mathbf{5 0 0 0}$ & $0.0130(0.0096)$ & $80.8923(1.0404)$ & $0.0129(0.0033)$ \\
\hline \multirow{6}{*}{$\mathbf{0 0} \%$} & $\mathbf{5 0 0}$ & $0.1275(0.1512)$ & $82.3122(6.2397)$ & $0.0279(0.0115)$ \\
& $\mathbf{2 0 0 0}$ & $0.0750(0.0966)$ & $81.0921(2.5078)$ & $0.0143(0.0038)$ \\
& $\mathbf{5 0 0 0}$ & $0.0532(0.0668)$ & $80.8686(1.5583)$ & $0.0094(0.0025)$ \\
\hline
\end{tabular}

Tabela A.10: estimativas e erros padrões das quantidades de interesse segundo o modelo de tempos de promoção para os pacientes submetidos ao tratamento A.

\begin{tabular}{lllll}
\hline Censura & $n$ & Prop. de cura & Tempo mediano & $\boldsymbol{d}_{\max }$ \\
\hline \multicolumn{2}{l}{ Verdadeiro valor } & 0.002 & 80.1706 & - \\
\hline$\approx \mathbf{5 \%}$ & $\mathbf{5 0 0}$ & $0.0012(0.0020)$ & $80.3891(2.2515)$ & $0.0453(0.0131)$ \\
& $\mathbf{2 0 0 0}$ & $0.0015(0.0012)$ & $80.2437(1.1554)$ & $0.0235(0.0064)$ \\
& $\mathbf{5 0 0 0}$ & $0.0017(0.0008)$ & $80.2203(0.7351)$ & $0.0150(0.0042)$ \\
\hline $\mathbf{2 0 \%}$ & $\mathbf{5 0 0}$ & $0.0194(0.0235)$ & $80.5003(2.4768)$ & $0.0436(0.0129)$ \\
& $\mathbf{2 0 0 0}$ & $0.0079(0.0123)$ & $80.2353(1.2285)$ & $0.0222(0.0065)$ \\
& $\mathbf{5 0 0 0}$ & $0.0029(0.0035)$ & $80.1500(0.7654)$ & $0.0141(0.0040)$ \\
\hline $\mathbf{6 0 \%}$ & $\mathbf{5 0 0}$ & $0.0390(0.0692)$ & $81.4859(4.5666)$ & $0.0340(0.0115)$ \\
& $\mathbf{2 0 0 0}$ & $0.0156(0.0311)$ & $80.5577(2.0967)$ & $0.0178(0.0057)$ \\
& $\mathbf{5 0 0 0}$ & $0.0010(0.0057)$ & $80.0707(1.0176)$ & $0.0117(0.0038)$ \\
\hline
\end{tabular}

Tabela A.11: estimativas e erros padrões das quantidades de interesse segundo o modelo proposto para os pacientes submetidos ao tratamento B.

\begin{tabular}{lllll}
\hline Censura & $\boldsymbol{n}$ & Prop. de cura & Tempo mediano & $\boldsymbol{d}_{\max }$ \\
\hline \multicolumn{2}{l}{ Verdadeiro valor } & 0.002 & 80.1706 & - \\
\hline$\approx \mathbf{5 \%}$ & $\mathbf{5 0 0}$ & $0.0032(0.0024)$ & $80.0000(2.9918)$ & $0.0406(0.0106)$ \\
& $\mathbf{2 0 0 0}$ & $0.0038(0.0014)$ & $79.9091(1.4582)$ & $0.0215(0.0054)$ \\
& $\mathbf{5 0 0 0}$ & $0.0041(0.0009)$ & $79.8569(0.9204)$ & $0.0146(0.0038)$ \\
\hline \multirow{2}{*}{$\mathbf{2 0} \%$} & $\mathbf{5 0 0}$ & $0.0231(0.0270)$ & $80.1079(3.1952)$ & $0.0374(0.0101)$ \\
& $\mathbf{2 0 0 0}$ & $0.0142(0.0143)$ & $79.9655(1.5960)$ & $0.0191(0.0049)$ \\
& $\mathbf{5 0 0 0}$ & $0.0119(0.0090)$ & $79.9714(0.9976)$ & $0.0125(0.0033)$ \\
\hline \multirow{6}{*0\%}{} & $\mathbf{5 0 0}$ & $0.1272(0.1510)$ & $82.1128(6.1237)$ & $0.0276(0.0116)$ \\
& $\mathbf{2 0 0 0}$ & $0.0740(0.0952)$ & $80.7298(2.4986)$ & $0.0145(0.0040)$ \\
& $\mathbf{5 0 0 0}$ & $0.0524(0.0662)$ & $80.5306(1.5432)$ & $0.0093(0.0024)$ \\
\hline
\end{tabular}

Tabela A.12: estimativas e erros padrões das quantidades de interesse segundo o modelo de tempos de promoção para os pacientes submetidos ao tratamento B. 


\begin{tabular}{lllllll}
\hline Censura & $\boldsymbol{n}$ & $\boldsymbol{\theta}_{\mathbf{1}}$ & $\boldsymbol{\theta}_{\mathbf{2}}$ & $\boldsymbol{\beta}_{\mathbf{0}}$ & $\boldsymbol{\beta}_{\mathbf{1}}$ & $\boldsymbol{\rho}$ \\
\hline \multicolumn{2}{l}{ Verdadeiro valor } & 2 & 0.005 & -8 & 2 & 5 \\
\hline $\mathbf{5 \%}$ & $\mathbf{5 0 0}$ & $1.9780(0.0782)$ & $0.0049(0.0003)$ & $-7.9682(2.2487)$ & $2.1797(1.5501)$ & $7.0481(4.5270)$ \\
& $\mathbf{2 0 0 0}$ & $1.9953(0.0372)$ & $0.0050(0.0001)$ & $-7.6764(1.1445)$ & $1.8881(0.6730)$ & $5.9703(1.7463)$ \\
& $\mathbf{5 0 0 0}$ & $1.9961(0.0240)$ & $0.0050(0.0001)$ & $-8.0573(0.7418)$ & $2.0539(0.4300)$ & $5.1208(0.9763)$ \\
\hline $\mathbf{2 0 \%}$ & $\mathbf{5 0 0}$ & $2.0019(0.1036)$ & $0.0050(0.0007)$ & $-8.7327(3.2964)$ & $2.8259(2.6481)$ & $6.3450(3.9910)$ \\
& $\mathbf{2 0 0 0}$ & $1.9911(0.0513)$ & $0.0051(0.0003)$ & $-11.0515(3.2114)$ & $4.4852(2.7770)$ & $4.0555(1.2810)$ \\
& $\mathbf{5 0 0 0}$ & $1.9947(0.0333)$ & $0.0051(0.0002)$ & $-9.7161(1.6328)$ & $3.2646(1.3380)$ & $4.0502(0.8017)$ \\
\hline \multirow{6}{*}{$\mathbf{5 0} \%$} & $\mathbf{5 0 0}$ & $2.0575(0.1743)$ & $0.0058(0.0029)$ & $-9.0290(2.6981)$ & $2.9516(2.1883)$ & $7.5017(7.3509)$ \\
& $\mathbf{2 0 0 0}$ & $2.0205(0.0944)$ & $0.0053(0.0017)$ & $-8.1368(1.7387)$ & $2.2177(1.2942)$ & $6.1299(3.4590)$ \\
& $\mathbf{5 0 0 0}$ & $2.0420(0.0857)$ & $0.0057(0.0017)$ & $-7.5967(1.8243)$ & $1.8885(1.2573)$ & $5.7212(2.4888)$ \\
\hline
\end{tabular}

Tabela A.13: estimativas e erros padrões dos parâmetros do modelo proposto.

\begin{tabular}{llllll}
\hline Censura & $\boldsymbol{n}$ & $\boldsymbol{\theta}_{\mathbf{1}}$ & $\boldsymbol{\theta}_{\mathbf{2}}$ & $\boldsymbol{\beta}_{\mathbf{0}}$ & $\boldsymbol{\beta}_{\mathbf{1}}$ \\
\hline$\approx \mathbf{5 \%}$ & $\mathbf{5 0 0}$ & $1.9773(0.0756)$ & $0.0048(0.0002)$ & $1.3130(0.0843)$ & $-0.6245(0.0947)$ \\
& $\mathbf{2 0 0 0}$ & $1.9932(0.0365)$ & $0.0049(0.0001)$ & $1.2912(0.0401)$ & $-0.6232(0.0477)$ \\
& $\mathbf{5 0 0 0}$ & $1.9965(0.0229)$ & $0.0049(0.0001)$ & $1.2841(0.0252)$ & $-0.6187(0.0307)$ \\
\hline $\mathbf{2 0 \%}$ & $\mathbf{5 0 0}$ & $2.0050(0.1046)$ & $0.0050(0.0006)$ & $1.2844(0.2452)$ & $-0.5995(0.1089)$ \\
& $\mathbf{2 0 0 0}$ & $2.0021(0.0526)$ & $0.0050(0.0003)$ & $1.2582(0.0944)$ & $-0.6022(0.0540)$ \\
& $\mathbf{5 0 0 0}$ & $2.0022(0.0344)$ & $0.0050(0.0002)$ & $1.2548(0.0526)$ & $-0.6021(0.0342)$ \\
\hline $\mathbf{6 0 \%}$ & $\mathbf{5 0 0}$ & $2.0606(0.1826)$ & $0.0054(0.0030)$ & $1.6134(1.4442)$ & $-0.5862(0.1556)$ \\
& $\mathbf{2 0 0 0}$ & $2.0196(0.1001)$ & $0.0051(0.0019)$ & $1.4042(0.8627)$ & $-0.5880(0.0766)$ \\
& $\mathbf{5 0 0 0}$ & $2.0096(0.0650)$ & $0.0051(0.0013)$ & $1.3155(0.5321)$ & $-0.5923(0.0485)$ \\
\hline
\end{tabular}

Tabela A.14: estimativas e erros padrões dos parâmetros do modelo de tempos de promoção.

\begin{tabular}{lllll}
\hline Censura & $\boldsymbol{n}$ & Prop. de cura & Tempo mediano & $\boldsymbol{d}_{\max }$ \\
\hline \multicolumn{2}{l}{ Verdadeiro valor } & 0.0245 & 113.6583 & - \\
\hline$\approx \mathbf{5 \%}$ & $\mathbf{5 0 0}$ & $0.0210(0.0085)$ & $113.8504(3.7544)$ & $0.0394(0.0096)$ \\
& $\mathbf{2 0 0 0}$ & $0.0237(0.0046)$ & $113.6223(1.8847)$ & $0.0202(0.0048)$ \\
& $\mathbf{5 0 0 0}$ & $0.0239(0.0029)$ & $113.7230(1.2744)$ & $0.0131(0.0040)$ \\
\hline $\mathbf{2 0} \%$ & $\mathbf{5 0 0}$ & $0.0233(0.0177)$ & $114.0111(3.9835)$ & $0.0375(0.0099)$ \\
& $\mathbf{2 0 0 0}$ & $0.0142(0.0118)$ & $114.0154(2.1086)$ & $0.0197(0.0075)$ \\
& $\mathbf{5 0 0 0}$ & $0.0172(0.0068)$ & $113.8436(1.2519)$ & $0.0125(0.0032)$ \\
\hline $\mathbf{6 0} \%$ & $\mathbf{5 0 0}$ & $0.0875(0.1048)$ & $114.0660(5.9308)$ & $0.0281(0.0081)$ \\
& $\mathbf{2 0 0 0}$ & $0.0536(0.0587)$ & $113.8171(2.7770)$ & $0.0146(0.0040)$ \\
& $\mathbf{5 0 0 0}$ & $0.0712(0.0733)$ & $114.0458(2.0057)$ & $0.0098(0.0038)$ \\
\hline
\end{tabular}

Tabela A.15: estimativas e erros padrões das quantidades de interesse segundo o modelo proposto para os pacientes submetidos ao tratamento A. 


\begin{tabular}{lllll}
\hline Censura & $\boldsymbol{n}$ & Prop. de cura & Tempo mediano & $\boldsymbol{d}_{\max }$ \\
\hline \multicolumn{2}{l}{ Verdadeiro valor } & 0.0245 & 113.6583 & - \\
\hline$\approx \mathbf{5 \%}$ & $\mathbf{5 0 0}$ & $0.0252(0.0077)$ & $113.1781(3.6848)$ & $0.0397(0.0098)$ \\
& $\mathbf{2 0 0 0}$ & $0.0265(0.0038)$ & $113.1147(1.8369)$ & $0.0209(0.0051)$ \\
& $\mathbf{5 0 0 0}$ & $0.0271(0.0025)$ & $113.1461(1.2044)$ & $0.0139(0.0036)$ \\
\hline $\mathbf{2 0 \%}$ & $\mathbf{5 0 0}$ & $0.0322(0.0170)$ & $113.6276(3.8857)$ & $0.0376(0.0102)$ \\
& $\mathbf{2 0 0 0}$ & $0.0306(0.0083)$ & $113.4943(1.8459)$ & $0.0197(0.0050)$ \\
& $\mathbf{5 0 0 0}$ & $0.0303(0.0054)$ & $113.4427(1.1877)$ & $0.0126(0.0031)$ \\
\hline $\mathbf{6 0 \%}$ & $\mathbf{5 0 0}$ & $0.1013(0.1123)$ & $114.4308(6.0193)$ & $0.0282(0.0081)$ \\
& $\mathbf{2 0 0 0}$ & $0.0638(0.0667)$ & $113.9511(2.8117)$ & $0.0147(0.0040)$ \\
& $\mathbf{5 0 0 0}$ & $0.0485(0.0455)$ & $113.7704(1.7664)$ & $0.0094(0.0026)$ \\
\hline
\end{tabular}

Tabela A.16: estimativas e erros padrões das quantidades de interesse segundo o modelo de tempos de promoção para os pacientes submetidos ao tratamento A.

\begin{tabular}{lllll}
\hline Censura & $\boldsymbol{n}$ & Prop. de cura & Tempo mediano & \multicolumn{1}{l}{$\boldsymbol{d}_{\max }$} \\
\hline \multicolumn{2}{l}{ Verdadeiro valor } & 0.1467 & 153.0664 & - \\
\hline$\approx \mathbf{5 \%}$ & $\mathbf{5 0 0}$ & $0.1416(0.0220)$ & $153.6900(6.5406)$ & $0.0376(0.0095)$ \\
& $\mathbf{2 0 0 0}$ & $0.1455(0.0110)$ & $153.4444(3.1689)$ & $0.0198(0.0050)$ \\
& $\mathbf{5 0 0 0}$ & $0.1463(0.0071)$ & $152.9650(2.0688)$ & $0.0125(0.0040)$ \\
\hline $\mathbf{2 0 \%}$ & $\mathbf{5 0 0}$ & $0.1442(0.0401)$ & $152.8830(6.8767)$ & $0.0351(0.0092)$ \\
& $\mathbf{2 0 0 0}$ & $0.1479(0.0196)$ & $153.0465(3.6091)$ & $0.0181(0.0056)$ \\
& $\mathbf{5 0 0 0}$ & $0.1479(0.0116)$ & $152.9920(2.2142)$ & $0.0116(0.0028)$ \\
\hline $\mathbf{6 0 \%}$ & $\mathbf{5 0 0}$ & $0.2181(0.1810)$ & $158.5090(14.2706)$ & $0.0251(0.0067)$ \\
& $\mathbf{2 0 0 0}$ & $0.1768(0.1270)$ & $154.7534(6.2520)$ & $0.0130(0.0033)$ \\
& $\mathbf{5 0 0 0}$ & $0.2111(0.1289)$ & $155.9872(5.2081)$ & $0.0096(0.0041)$ \\
\hline
\end{tabular}

Tabela A.17: estimativas e erros padrões das quantidades de interesse segundo o modelo proposto para os pacientes submetidos ao tratamento B.

\begin{tabular}{lllll}
\hline Censura & $\boldsymbol{n}$ & Prop. de cura & Tempo mediano & \multicolumn{1}{l}{$\boldsymbol{d}_{\max }$} \\
\hline \multicolumn{2}{l}{ Verdadeiro valor } & 0.1467 & 153.0664 & - \\
\hline$\approx \mathbf{5 \%}$ & $\mathbf{5 0 0}$ & $0.1374(0.0217)$ & $154.8944(6.4656)$ & $0.0394(0.0104)$ \\
& $\mathbf{2 0 0 0}$ & $0.1424(0.0108)$ & $154.3891(3.1925)$ & $0.0210(0.0056)$ \\
& $\mathbf{5 0 0 0}$ & $0.1430(0.0068)$ & $154.0204(2.0144)$ & $0.0141(0.0036)$ \\
\hline $\mathbf{2 0} \%$ & $\mathbf{5 0 0}$ & $0.1435(0.0399)$ & $153.2500(6.7982)$ & $0.0353(0.0092)$ \\
& $\mathbf{2 0 0 0}$ & $0.1464(0.0195)$ & $153.3149(3.5092)$ & $0.0184(0.0047)$ \\
& $\mathbf{5 0 0 0}$ & $0.1468(0.0121)$ & $153.2141(2.2115)$ & $0.0119(0.0030)$ \\
\hline $\mathbf{6 0} \%$ & $\mathbf{5 0 0}$ & $0.2105(0.1810)$ & $157.9965(14.1504)$ & $0.0252(0.0067)$ \\
& $\mathbf{2 0 0 0}$ & $0.1746(0.1314)$ & $154.5164(6.2196)$ & $0.0131(0.0033)$ \\
& $\mathbf{5 0 0 0}$ & $0.1600(0.1011)$ & $153.6541(3.7453)$ & $0.0083(0.0020)$ \\
\hline
\end{tabular}

Tabela A.18: estimativas e erros padrões das quantidades de interesse segundo o modelo de tempos de promoção para os pacientes submetidos ao tratamento B. 


\begin{tabular}{lllllll}
\hline Censura & $\boldsymbol{n}$ & $\boldsymbol{\theta}_{\mathbf{1}}$ & $\boldsymbol{\theta}_{\mathbf{2}}$ & $\boldsymbol{\beta}_{\mathbf{0}}$ & $\boldsymbol{\beta}_{\mathbf{1}}$ & $\boldsymbol{\rho}$ \\
\hline \multicolumn{2}{l}{ Verdadeiro valor } & 2 & 0.005 & -8 & 2 & 5 \\
\hline$\approx \mathbf{5 \%}$ & $\mathbf{5 0 0}$ & $1.9738(0.0816)$ & $0.0050(0.0011)$ & $-11.0964(4.6228)$ & $3.8535(4.2562)$ & $5.3016(1.9190)$ \\
& $\mathbf{2 0 0 0}$ & $1.9884(0.0395)$ & $0.0049(0.0004)$ & $-8.8888(2.5383)$ & $2.7850(2.4812)$ & $5.2328(0.7270)$ \\
& $\mathbf{5 0 0 0}$ & $1.9927(0.0279)$ & $0.0049(0.0002)$ & $-8.3395(1.2675)$ & $2.1107(1.2335)$ & $5.1219(0.4314)$ \\
\hline $\mathbf{2 0} \%$ & $\mathbf{5 0 0}$ & $1.9996(0.1060)$ & $0.0080(0.0022)$ & $-9.7981(3.3863)$ & $2.8543(3.4528)$ & $2.2016(2.4089)$ \\
& $\mathbf{2 0 0 0}$ & $2.0115(0.0611)$ & $0.0076(0.0020)$ & $-9.1353(2.1607)$ & $2.4461(2.3086)$ & $2.4325(2.0444)$ \\
& $\mathbf{5 0 0 0}$ & $1.9909(0.0506)$ & $0.0049(0.0012)$ & $-9.3080(3.1859)$ & $2.9806(3.1493)$ & $5.8878(2.5610)$ \\
\hline \multirow{6}{*}{$\mathbf{0 0} \%$} & $\mathbf{5 0 0}$ & $2.0030(0.1357)$ & $0.0062(0.0024)$ & $-9.3163(3.9895)$ & $2.5610(4.3398)$ & $5.4540(6.0265)$ \\
& $\mathbf{2 0 0 0}$ & $2.0067(0.0677)$ & $0.0055(0.0016)$ & $-8.7478(2.5463)$ & $2.5362(2.5310)$ & $5.1544(3.6852)$ \\
& $\mathbf{5 0 0 0}$ & $1.9856(0.0430)$ & $0.0041(0.0010)$ & $-8.4654(2.0130)$ & $2.4319(1.9529)$ & $7.7220(2.0701)$ \\
\hline
\end{tabular}

Tabela A.19: estimativas e erros padrões dos parâmetros do modelo proposto.

\begin{tabular}{llllll}
\hline Censura & $\boldsymbol{n}$ & $\boldsymbol{\theta}_{\mathbf{1}}$ & $\boldsymbol{\theta}_{\mathbf{2}}$ & $\boldsymbol{\beta}_{\mathbf{0}}$ & $\boldsymbol{\beta}_{\mathbf{1}}$ \\
\hline $\boldsymbol{\mathbf { 5 } \%}$ & $\mathbf{5 0 0}$ & $1.9444(0.0774)$ & $0.0043(0.0005)$ & $1.8100(0.1825)$ & $-0.1080(0.0915)$ \\
& $\mathbf{2 0 0 0}$ & $1.9947(1.0439)$ & $0.0045(0.0003)$ & $2.1119(12.8252)$ & $-0.3000(10.6641)$ \\
& $\mathbf{5 0 0 0}$ & $1.9676(0.0249)$ & $0.0046(0.0001)$ & $1.7119(0.0401)$ & $-0.1069(0.0288)$ \\
\hline $\mathbf{2 0 \%}$ & $\mathbf{5 0 0}$ & $2.0124(0.1331)$ & $0.0048(0.0019)$ & $1.8843(0.9649)$ & $-0.0868(0.0990)$ \\
& $\mathbf{2 0 0 0}$ & $1.9955(0.0678)$ & $0.0047(0.0011)$ & $1.7151(0.4360)$ & $-0.0905(0.0503)$ \\
& $\mathbf{5 0 0 0}$ & $1.9964(0.0443)$ & $0.0048(0.0007)$ & $1.6520(0.2512)$ & $-0.0897(0.0323)$ \\
\hline $\mathbf{6 0 \%}$ & $\mathbf{5 0 0}$ & $2.0753(0.1913)$ & $0.0061(0.0043)$ & $1.8605(1.6026)$ & $-0.0791(0.1358)$ \\
& $\mathbf{2 0 0 0}$ & $2.0296(0.0944)$ & $0.0051(0.0029)$ & $1.9346(1.1907)$ & $-0.0756(0.0701)$ \\
& $\mathbf{5 0 0 0}$ & $2.0123(0.0663)$ & $0.0046(0.0023)$ & $2.0087(0.9590)$ & $-0.0772(0.0459)$ \\
\hline
\end{tabular}

Tabela A.20: estimativas e erros padrões dos parâmetros do modelo de tempos de promoção.

\begin{tabular}{lllll}
\hline Censura & $\boldsymbol{n}$ & Prop. de cura & Tempo mediano & $\boldsymbol{d}_{\max }$ \\
\hline \multicolumn{2}{l}{ Verdadeiro valor } & 0.0021 & 82.1341 & - \\
\hline$\approx \mathbf{5 \%}$ & $\mathbf{5 0 0}$ & $0.0013(0.0017)$ & $82.3986(2.7096)$ & $0.0450(0.0125)$ \\
& $\mathbf{2 0 0 0}$ & $0.0016(0.0010)$ & $82.2141(1.3199)$ & $0.0229(0.0061)$ \\
& $\mathbf{5 0 0 0}$ & $0.0018(0.0007)$ & $82.1681(0.8779)$ & $0.0149(0.0042)$ \\
\hline \multirow{2}{*}{$\mathbf{2 0}$} & $\mathbf{5 0 0}$ & $0.0189(0.0217)$ & $82.5966(2.7647)$ & $0.0420(0.0118)$ \\
& $\mathbf{2 0 0 0}$ & $0.0139(0.0132)$ & $82.3329(1.4665)$ & $0.0218(0.0060)$ \\
& $\mathbf{5 0 0 0}$ & $0.0027(0.0039)$ & $82.2513(1.1214)$ & $0.0144(0.0045)$ \\
\hline \multirow{6}{*}{$\mathbf{0 0} \%$} & $\mathbf{5 0 0}$ & $0.0183(0.0332)$ & $82.9190(3.9959)$ & $0.0335(0.0104)$ \\
& $\mathbf{2 0 0 0}$ & $0.0075(0.0120)$ & $82.4226(2.0602)$ & $0.0171(0.0051)$ \\
& $\mathbf{5 0 0 0}$ & $0.0014(0.0051)$ & $82.0601(1.2360)$ & $0.0110(0.0033)$ \\
\hline
\end{tabular}

Tabela A.21: estimativas e erros padrões das quantidades de interesse segundo o modelo proposto para os pacientes submetidos ao tratamento A. 


\begin{tabular}{lllll}
\hline Censura & $\boldsymbol{n}$ & Prop. de cura & Tempo mediano & $\boldsymbol{d}_{\max }$ \\
\hline \multicolumn{2}{l}{ Verdadeiro valor } & 0.0021 & 82.1341 & - \\
\hline$\approx \mathbf{5 \%}$ & $\mathbf{5 0 0}$ & $0.0031(0.0024)$ & $82.0556(3.0632)$ & $0.0410(0.0105)$ \\
& $\mathbf{2 0 0 0}$ & $0.0037(0.0013)$ & $81.9209(2.9051)$ & $0.0222(0.0362)$ \\
& $\mathbf{5 0 0 0}$ & $0.0040(0.0009)$ & $81.9038(0.9511)$ & $0.0137(0.0035)$ \\
\hline \multirow{2}{*}{$\mathbf{2 0} \%$} & $\mathbf{5 0 0}$ & $0.0198(0.0246)$ & $82.0935(3.2374)$ & $0.0370(0.0096)$ \\
& $\mathbf{2 0 0 0}$ & $0.0102(0.0112)$ & $82.0521(1.6389)$ & $0.0193(0.0048)$ \\
& $\mathbf{5 0 0 0}$ & $0.0085(0.0072)$ & $82.0605(1.0866)$ & $0.0123(0.0030)$ \\
\hline \multirow{6}{*}{$\mathbf{0 0} \%$} & $\mathbf{5 0 0}$ & $0.1093(0.1427)$ & $83.3195(5.8918)$ & $0.0284(0.0080)$ \\
& $\mathbf{2 0 0 0}$ & $0.0515(0.0796)$ & $82.3283(2.4008)$ & $0.0146(0.0039)$ \\
& $\mathbf{5 0 0 0}$ & $0.0298(0.0588)$ & $82.0410(1.5517)$ & $0.0096(0.0026)$ \\
\hline
\end{tabular}

Tabela A.22: estimativas e erros padrões das quantidades de interesse segundo o modelo de tempos de promoção para os pacientes submetidos ao tratamento A.

\begin{tabular}{lllll}
\hline Censura & $\boldsymbol{n}$ & Prop. de cura & Tempo mediano & $\boldsymbol{d}_{\max }$ \\
\hline \multicolumn{2}{l}{ Verdadeiro valor } & 0.0068 & 86.0528 & - \\
\hline$\approx \mathbf{5 \%}$ & $\mathbf{5 0 0}$ & $0.0053(0.0042)$ & $86.2073(2.9461)$ & $0.0453(0.0124)$ \\
& $\mathbf{2 0 0 0}$ & $0.0059(0.0022)$ & $86.2595(1.4289)$ & $0.0236(0.0063)$ \\
& $\mathbf{5 0 0 0}$ & $0.0062(0.0016)$ & $86.1242(0.9559)$ & $0.0156(0.0040)$ \\
\hline $\mathbf{2 0} \%$ & $\mathbf{5 0 0}$ & $0.0427(0.0324)$ & $85.8490(2.9520)$ & $0.0423(0.0120)$ \\
& $\mathbf{2 0 0 0}$ & $0.0373(0.0245)$ & $85.7739(1.4897)$ & $0.0219(0.0060)$ \\
& $\mathbf{5 0 0 0}$ & $0.0088(0.0104)$ & $86.1643(1.1071)$ & $0.0151(0.0045)$ \\
\hline $\mathbf{6 0} \%$ & $\mathbf{5 0 0}$ & $0.0390(0.0534)$ & $86.6313(4.7398)$ & $0.0338(0.0106)$ \\
& $\mathbf{2 0 0 0}$ & $0.0203(0.0260)$ & $86.2520(2.3156)$ & $0.0181(0.0056)$ \\
& $\mathbf{5 0 0 0}$ & $0.0045(0.0136)$ & $85.8836(1.4231)$ & $0.0125(0.0040)$ \\
\hline
\end{tabular}

Tabela A.23: estimativas e erros padrões das quantidades de interesse segundo o modelo proposto para os pacientes submetidos ao tratamento B.

\begin{tabular}{lllll}
\hline Censura & $\boldsymbol{n}$ & Prop. de cura & Tempo mediano & $\boldsymbol{d}_{\max }$ \\
\hline \multicolumn{2}{l}{ Verdadeiro valor } & 0.0068 & 86.0528 & - \\
\hline$\approx \mathbf{5 \%}$ & $\mathbf{5 0 0}$ & $0.0056(0.0040)$ & $86.8874(3.2452)$ & $0.0426(0.0110)$ \\
& $\mathbf{2 0 0 0}$ & $0.0065(0.0022)$ & $86.6531(3.0871)$ & $0.0244(0.0359)$ \\
& $\mathbf{5 0 0 0}$ & $0.0070(0.0014)$ & $86.6491(1.0582)$ & $0.0161(0.0041)$ \\
\hline \multirow{2}{*}{$\mathbf{2 0} \%$} & $\mathbf{5 0 0}$ & $0.0255(0.0299)$ & $85.9015(3.4966)$ & $0.0373(0.0098)$ \\
& $\mathbf{2 0 0 0}$ & $0.0145(0.0147)$ & $86.0249(1.7491)$ & $0.0198(0.0051)$ \\
& $\mathbf{5 0 0 0}$ & $0.0123(0.0097)$ & $85.9983(1.1563)$ & $0.0131(0.0034)$ \\
\hline \multirow{6}{*}{$\mathbf{0 0} \%$} & $\mathbf{5 0 0}$ & $0.1201(0.1518)$ & $87.1697(6.3347)$ & $0.0279(0.0079)$ \\
& $\mathbf{2 0 0 0}$ & $0.0592(0.0881)$ & $85.7190(2.5681)$ & $0.0147(0.0037)$ \\
& $\mathbf{5 0 0 0}$ & $0.0349(0.0650)$ & $85.4145(1.6565)$ & $0.0099(0.0027)$ \\
\hline
\end{tabular}

Tabela A.24: estimativas e erros padrões das quantidades de interesse segundo o modelo de tempos de promoção para os pacientes submetidos ao tratamento B. 


\section{Apêndice B}

\section{Gráficos}

A tabela seguinte tem como objetivo auxiliar a análise dos resultados obtidos nos estudos de simulação. Nela são listados o número e página de cada gráfico do tipo boxplot construído a partir das estimativas obtidas para os parâmetros do modelo proposto e das quantidades de interesse calculadas para cada configuração de amostra e tipo de tratamento.

\begin{tabular}{cccc}
\hline Configuração & Parâmetro & Gráfico & Página \\
\hline \multirow{3}{*}{ I } & $\theta_{1}$ & B.1 & 61 \\
& $\theta_{2}$ & B.2 & 61 \\
& $\beta_{0}$ & B.3 & 61 \\
& $\beta_{1}$ & B.4 & 61 \\
$\rho$ & B.5 & 61 \\
\hline \multirow{4}{*}{ II } & $\theta_{1}$ & B.6 & 62 \\
& $\theta_{2}$ & B.7 & 62 \\
& $\beta_{0}$ & B.8 & 62 \\
& $\beta_{1}$ & B.9 & 62 \\
& $\rho$ & B.10 & 62 \\
\hline III & $\theta_{1}$ & B.11 & 63 \\
& $\theta_{2}$ & B.12 & 63 \\
& $\beta_{0}$ & B.13 & 63 \\
& $\beta_{1}$ & B.14 & 63 \\
& $\rho$ & B.15 & 63 \\
\hline \multirow{4}{*}{ IV } & $\theta_{1}$ & B.16 & 64 \\
& $\theta_{2}$ & B.17 & 64 \\
& $\beta_{0}$ & B.18 & 64 \\
& $\beta_{1}$ & B.19 & 64 \\
& $\rho$ & B.20 & 64 \\
\hline
\end{tabular}




\begin{tabular}{|c|c|c|c|c|c|}
\hline Configuração & Tratamento & Estimativa & Modelo & Gráfico & Página \\
\hline \multirow{12}{*}{ I } & \multirow{6}{*}{ A } & \multirow{2}{*}{ cura } & nascimento e morte & B.21 & 65 \\
\hline & & & tempos de promoção & B.22 & 65 \\
\hline & & \multirow{2}{*}{ tempo mediano } & nascimento e morte & B.23 & 65 \\
\hline & & & tempos de promoção & B.24 & 65 \\
\hline & & \multirow{2}{*}{ distância máxima } & nascimento e morte & B.25 & 65 \\
\hline & & & tempos de promoção & B.26 & 65 \\
\hline & \multirow{6}{*}{ B } & \multirow{2}{*}{ cura } & nascimento e morte & B.27 & 66 \\
\hline & & & tempos de promoção & B.28 & 66 \\
\hline & & \multirow{2}{*}{ tempo mediano } & nascimento e morte & B.29 & 66 \\
\hline & & & tempos de promoção & B.30 & 66 \\
\hline & & \multirow{2}{*}{ distância máxima } & nascimento e morte & B.31 & 66 \\
\hline & & & tempos de promoção & B.32 & 66 \\
\hline \multirow{12}{*}{ II } & \multirow{6}{*}{ A } & \multirow{2}{*}{ cura } & nascimento e morte & B.33 & 67 \\
\hline & & & tempos de promoção & B.34 & 67 \\
\hline & & \multirow{2}{*}{ tempo mediano } & nascimento e morte & B.35 & 67 \\
\hline & & & tempos de promoção & B.36 & 67 \\
\hline & & \multirow{2}{*}{ distância máxima } & nascimento e morte & B.37 & 67 \\
\hline & & & tempos de promoção & B.38 & 67 \\
\hline & \multirow{6}{*}{ B } & \multirow{2}{*}{ cura } & nascimento e morte & B.39 & 68 \\
\hline & & & tempos de promoção & B.40 & 68 \\
\hline & & \multirow{2}{*}{ tempo mediano } & nascimento e morte & B.41 & 68 \\
\hline & & & tempos de promoção & B.42 & 68 \\
\hline & & \multirow{2}{*}{ distância máxima } & nascimento e morte & B.43 & 68 \\
\hline & & & tempos de promoção & B.44 & 68 \\
\hline \multirow{12}{*}{ III } & \multirow{6}{*}{ A } & \multirow{2}{*}{ cura } & nascimento e morte & B. 45 & 69 \\
\hline & & & tempos de promoção & B.46 & 69 \\
\hline & & \multirow{2}{*}{ tempo mediano } & nascimento e morte & B.47 & 69 \\
\hline & & & tempos de promoção & B.48 & 69 \\
\hline & & distância máxima & nascimento e morte & B.49 & 69 \\
\hline & & ustancta maxmin & tempos de promoção & B.50 & 69 \\
\hline & & cura & nascimento e morte & B.51 & 70 \\
\hline & & & tempos de promoção & B.52 & 70 \\
\hline & $B$ & tempo mediano & nascimento e morte & B.53 & 70 \\
\hline & & & tempos de promoção & B.54 & 70 \\
\hline & & distância máxima & nascimento e morte & B.55 & 70 \\
\hline & & aistancia maxima & tempos de promoção & B.56 & 70 \\
\hline & & cura $\mathrm{r}$, & nascimento e morte & B.57 & 71 \\
\hline & & Culd & tempos de promoção & B.58 & 71 \\
\hline & A & temno mediano & nascimento e morte & B.59 & 71 \\
\hline & $\Lambda$ & Lempo inecirano & tempos de promoção & B.60 & 71 \\
\hline & & distância máxima & nascimento e morte & B.61 & 71 \\
\hline IV & & & tempos de promoção & B.62 & 71 \\
\hline & & cura & nascimento e morte & B.63 & 72 \\
\hline & & Culd & tempos de promoção & B.64 & 72 \\
\hline & $B$ & temno mediano & nascimento e morte & B.65 & 72 \\
\hline & D & . & tempos de promoção & B.66 & 72 \\
\hline & & distância máxima & nascimento e morte & B.67 & 72 \\
\hline & & Gistancla minasinta & tempos de promoção & B.68 & 72 \\
\hline
\end{tabular}




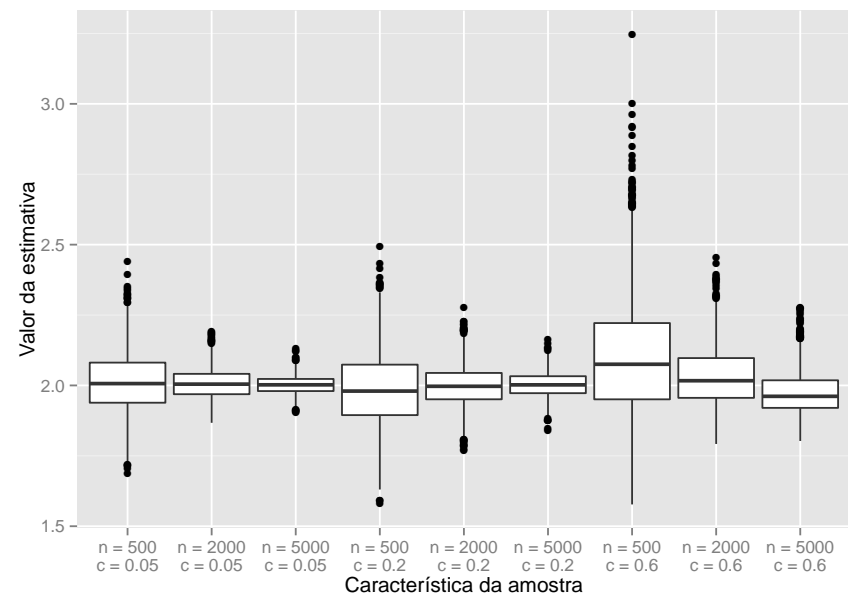

Figura B.1: distribuição das estimativas de $\theta_{1}$.

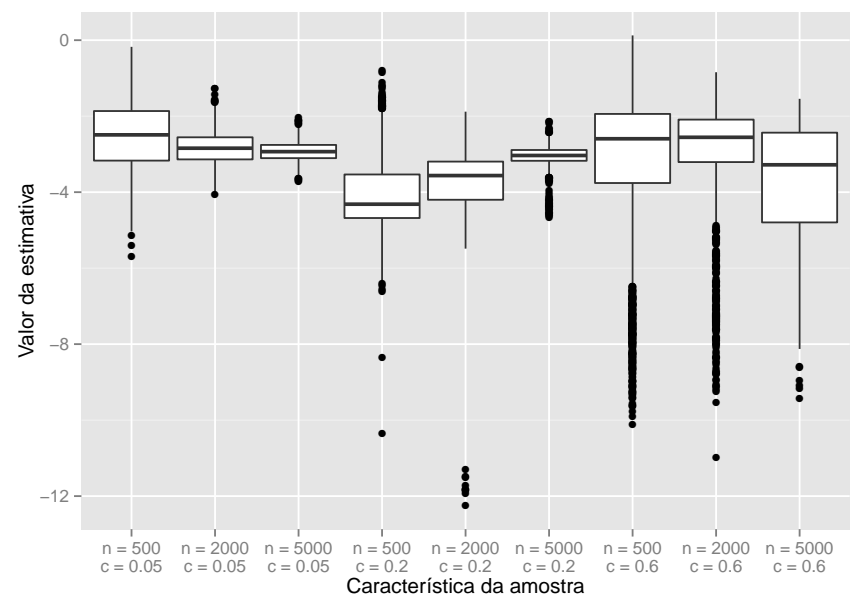

Figura B.3: distribuição das estimativas de $\beta_{0}$.

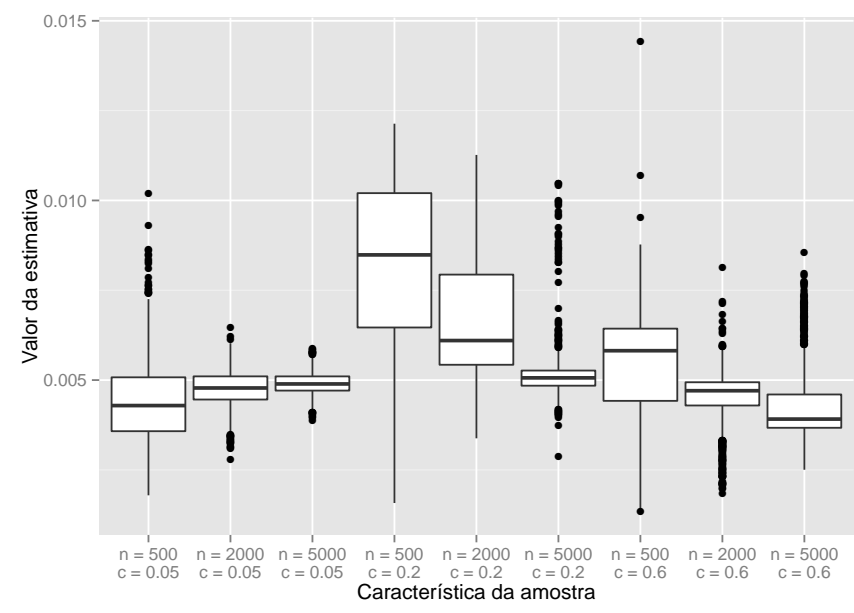

Figura B.2: distribuição das estimativas de $\theta_{2}$.

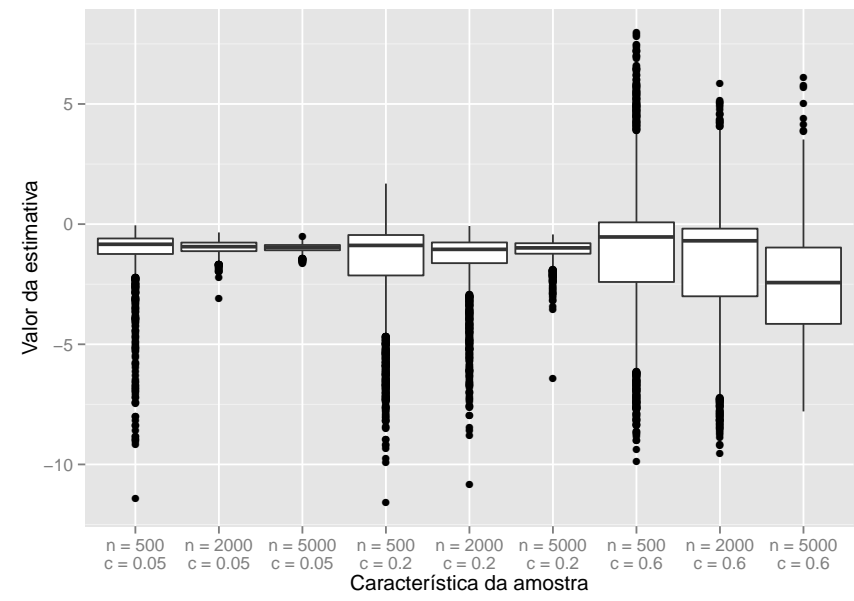

Figura B.4: distribuição das estimativas de $\beta_{1}$.

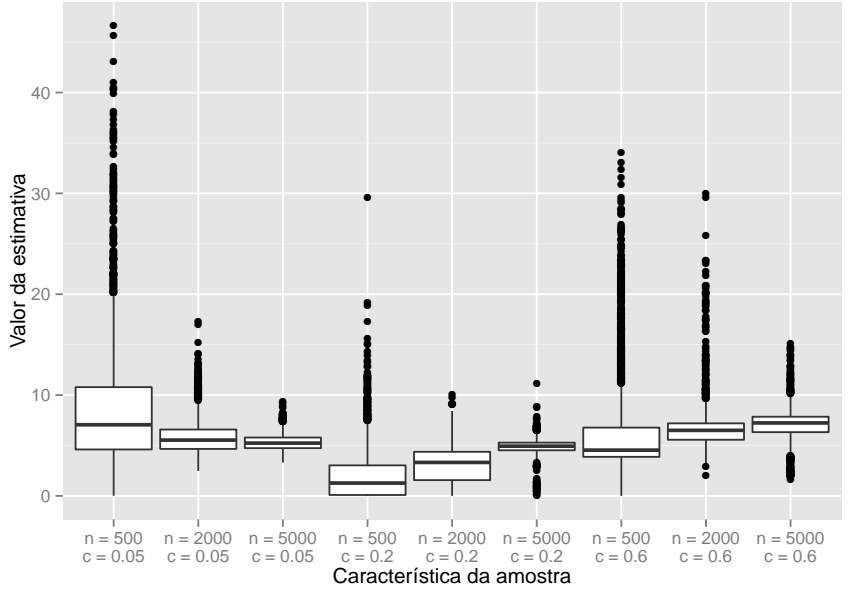

Figura B.5: distribuição das estimativas de $\rho$. 


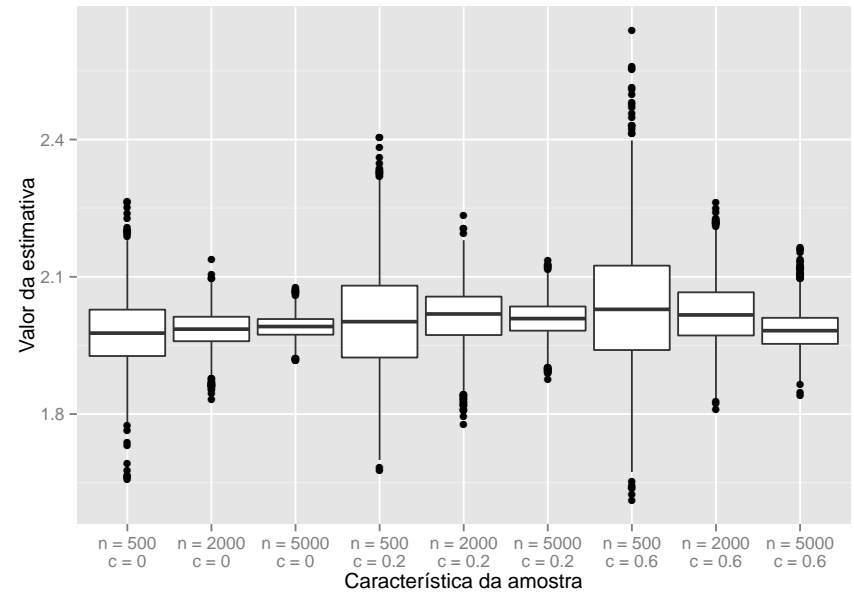

Figura B.6: distribuição das estimativas de $\theta_{1}$.

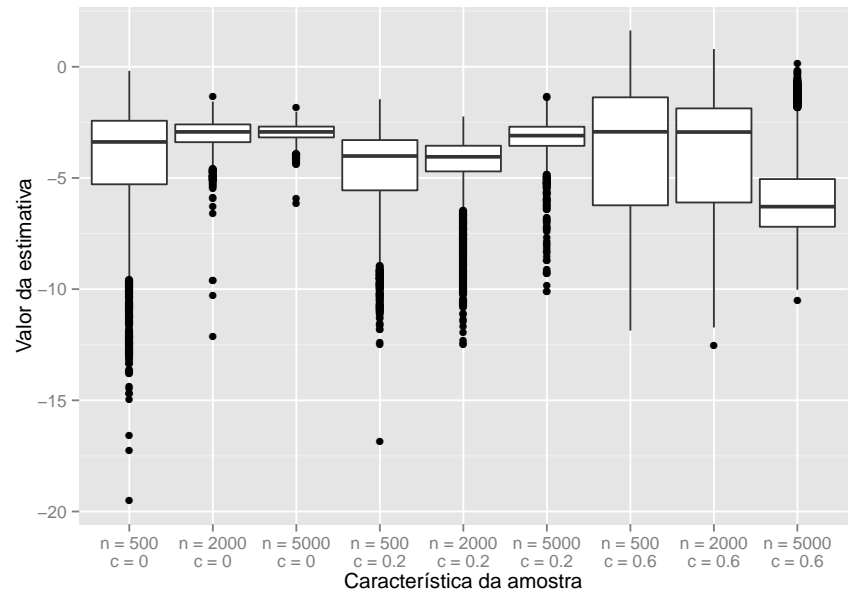

Figura B.8: distribuição das estimativas de $\beta_{0}$.

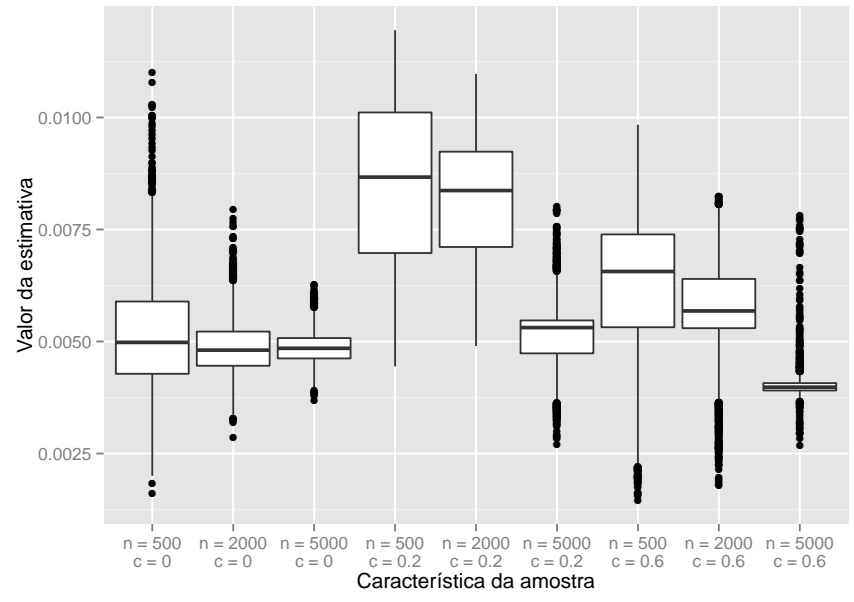

Figura B.7: distribuição das estimativas de $\theta_{2}$.

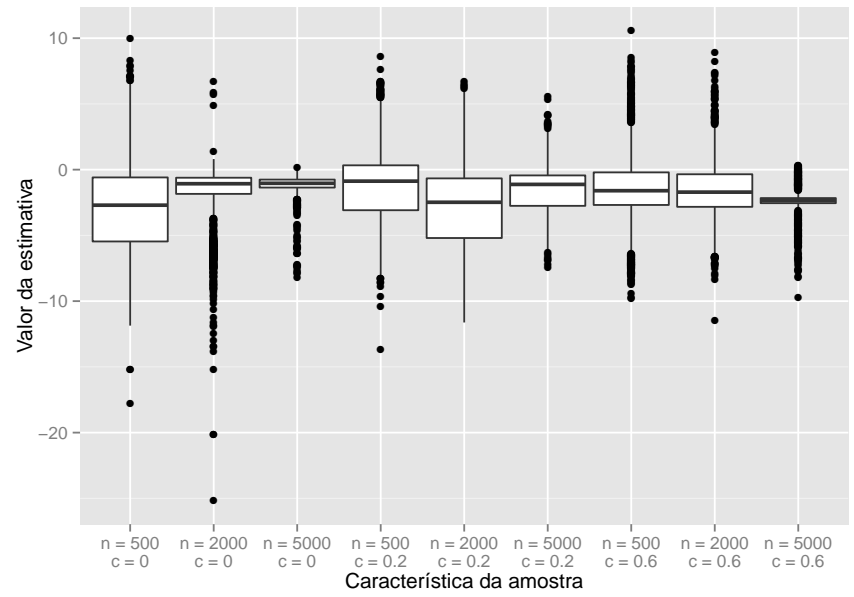

Figura B.9: distribuição das estimativas de $\beta_{1}$.

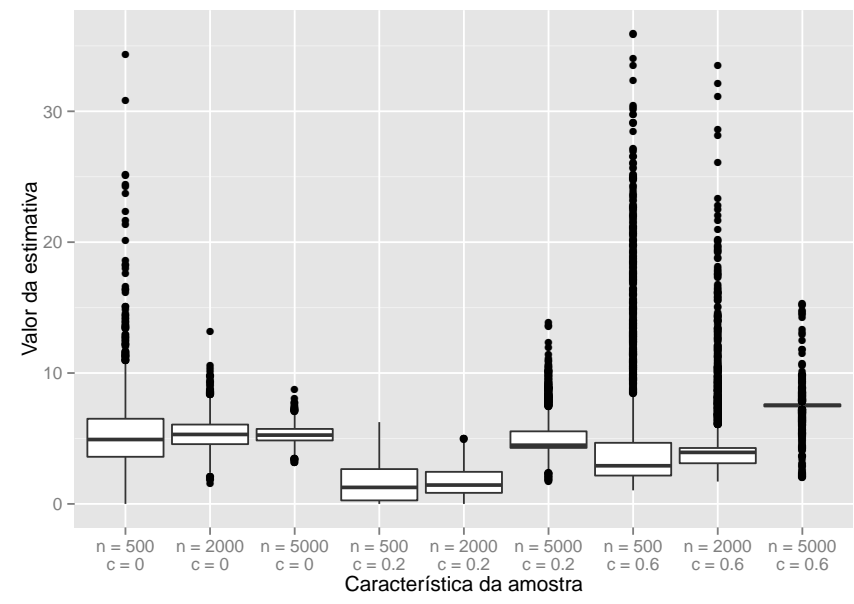

Figura B.10: distribuição das estimativas de $\rho$. 


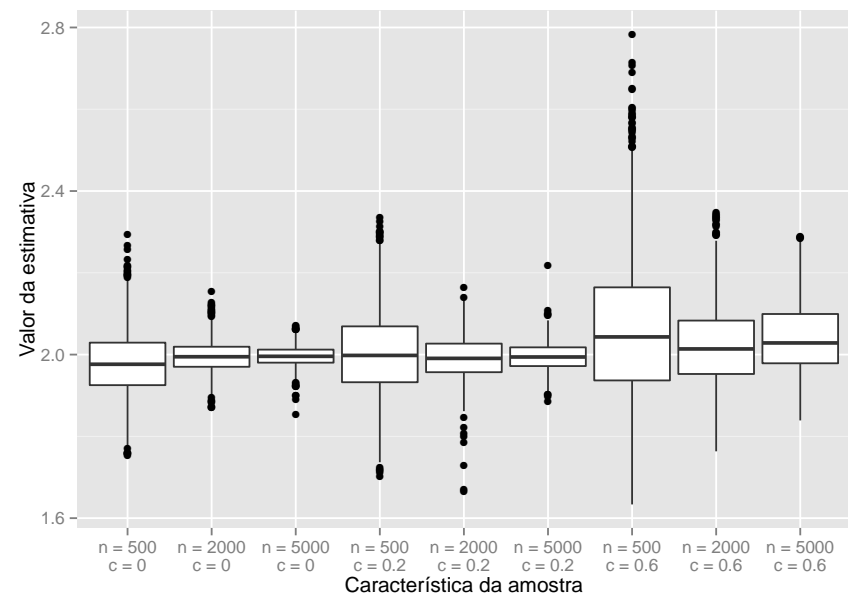

Figura B.11: distribuição das estimativas de $\theta_{1}$.

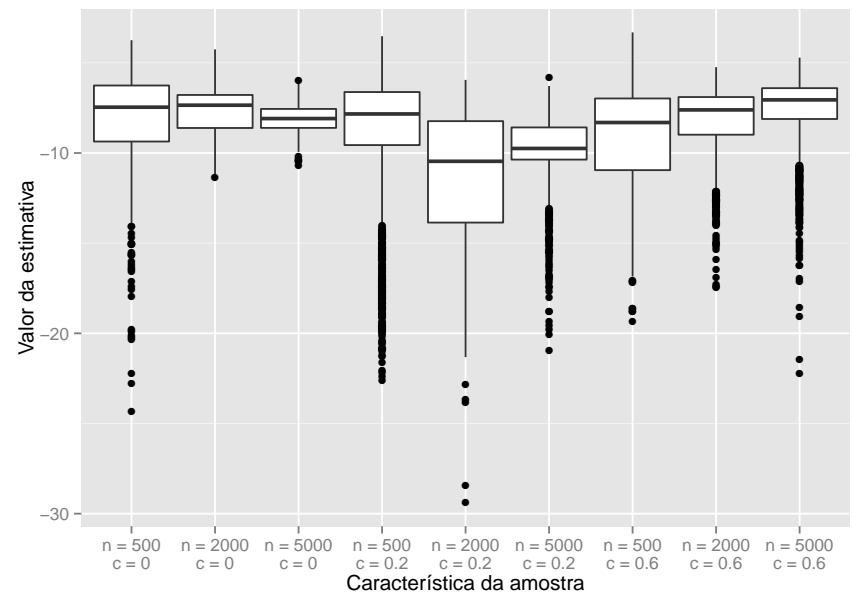

Figura B.13: distribuição das estimativas de $\beta_{0}$.

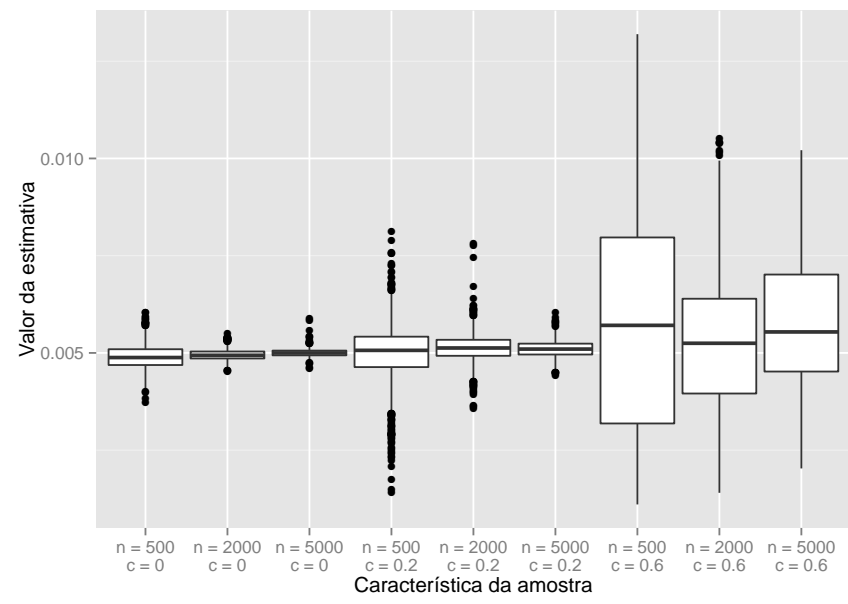

Figura B.12: distribuição das estimativas de $\theta_{2}$.

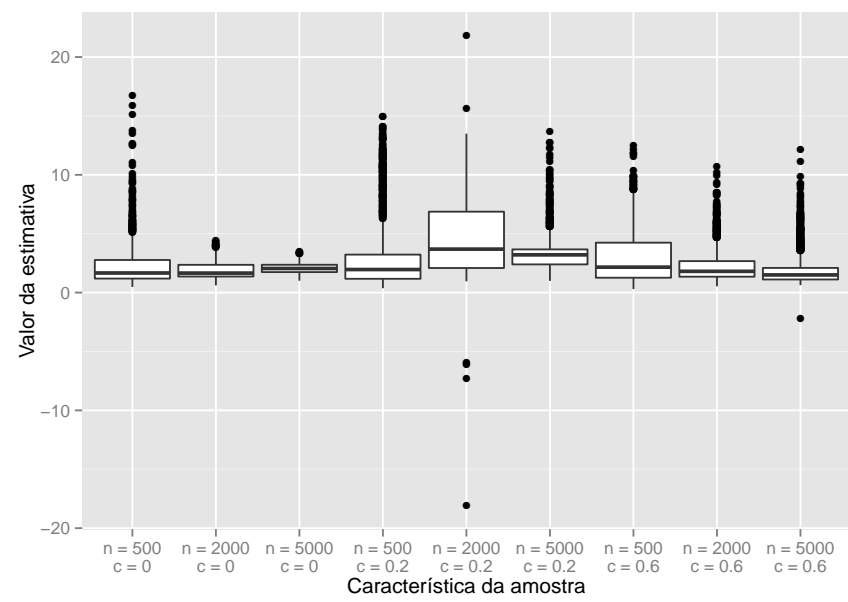

Figura B.14: distribuição das estimativas de $\beta_{1}$.

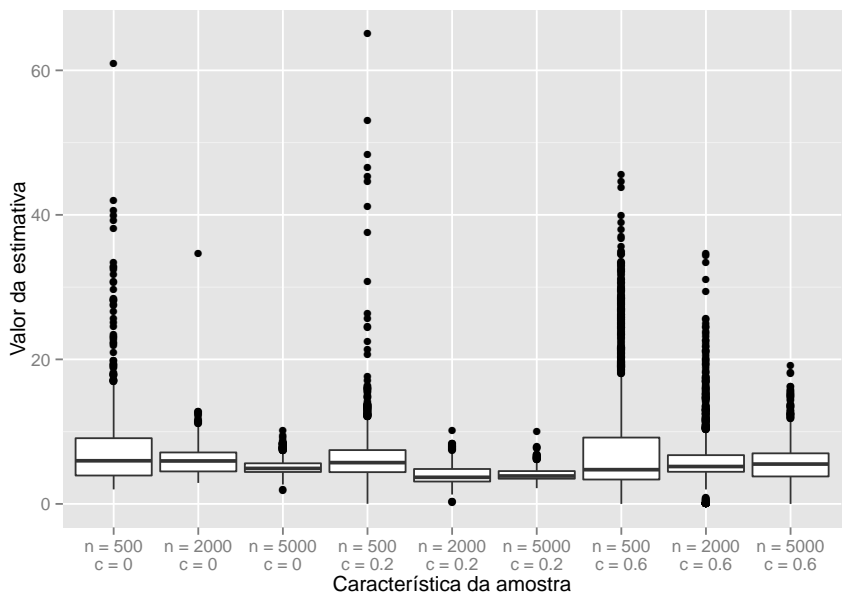

Figura B.15: distribuição das estimativas de $\rho$. 


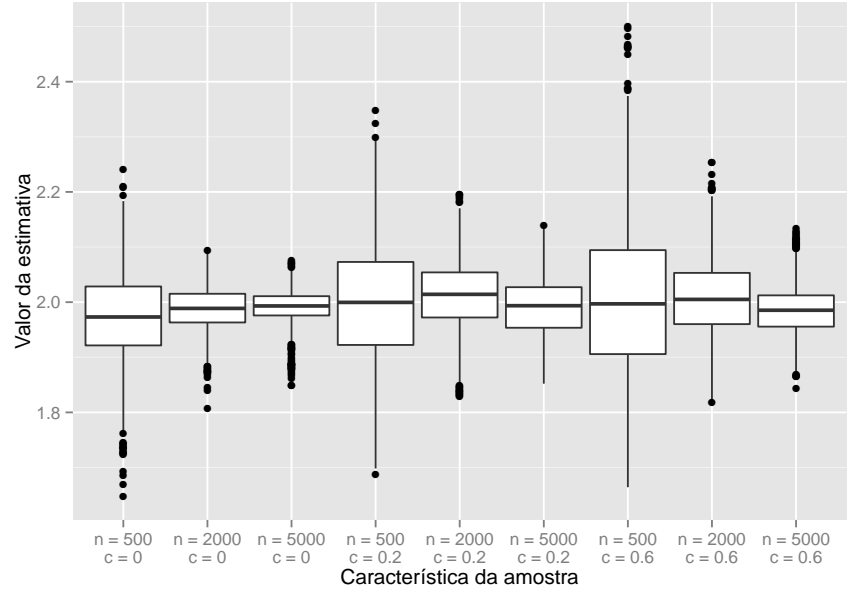

Figura B.16: distribuição das estimativas de $\theta_{1}$.

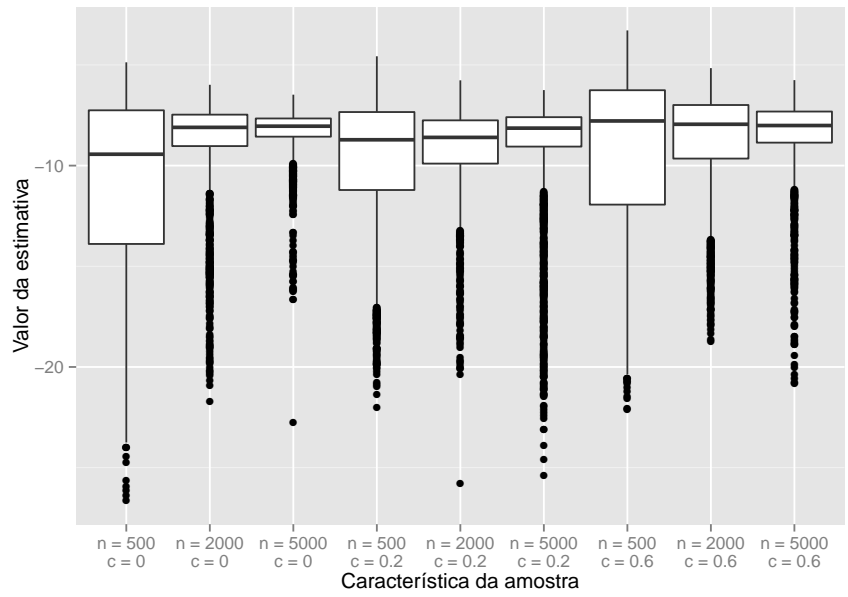

Figura B.18: distribuição das estimativas de $\beta_{0}$.

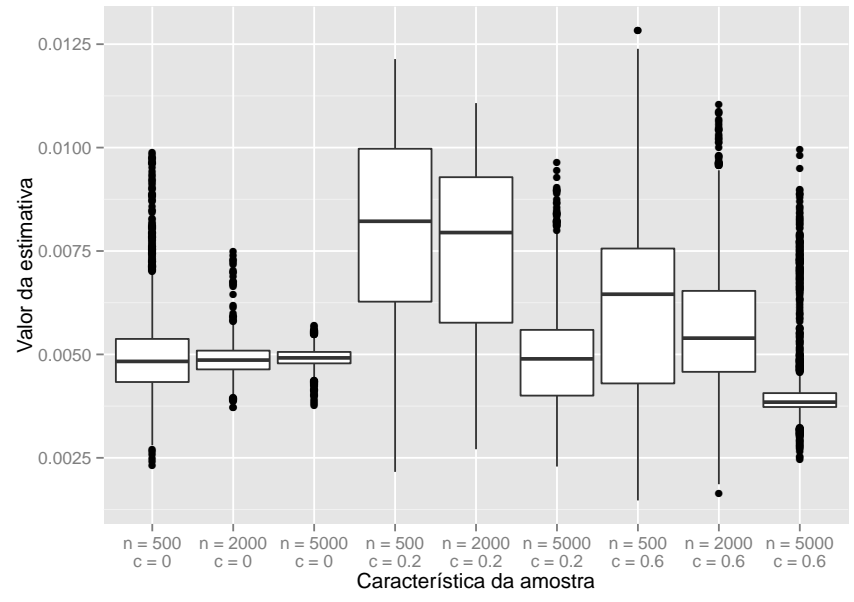

Figura B.17: distribuição das estimativas de $\theta_{2}$.

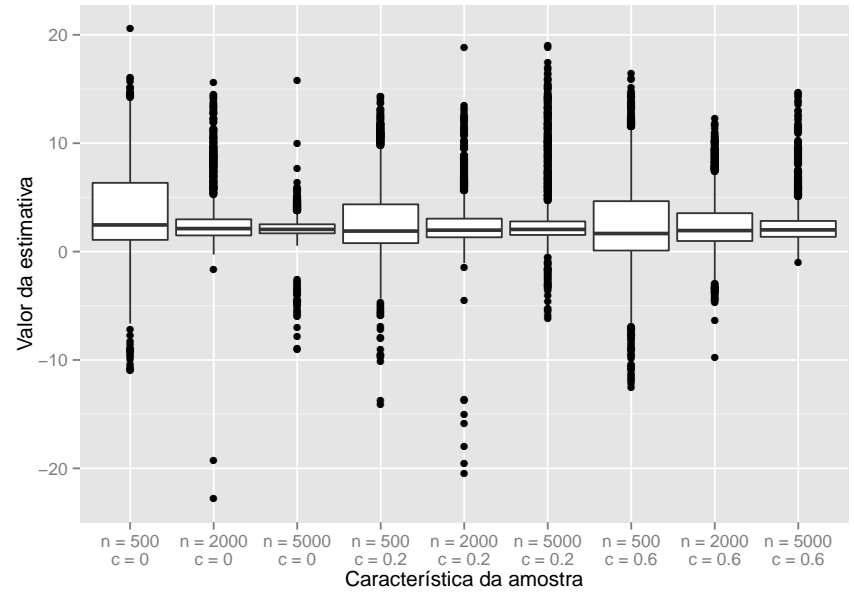

Figura B.19: distribuição das estimativas de $\beta_{1}$.

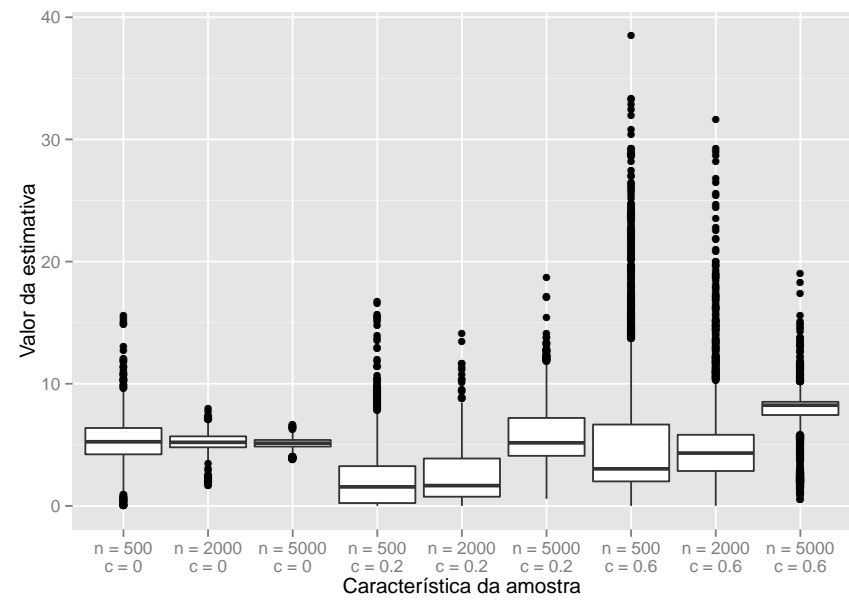

Figura B.20: distribuição das estimativas de $\rho$. 


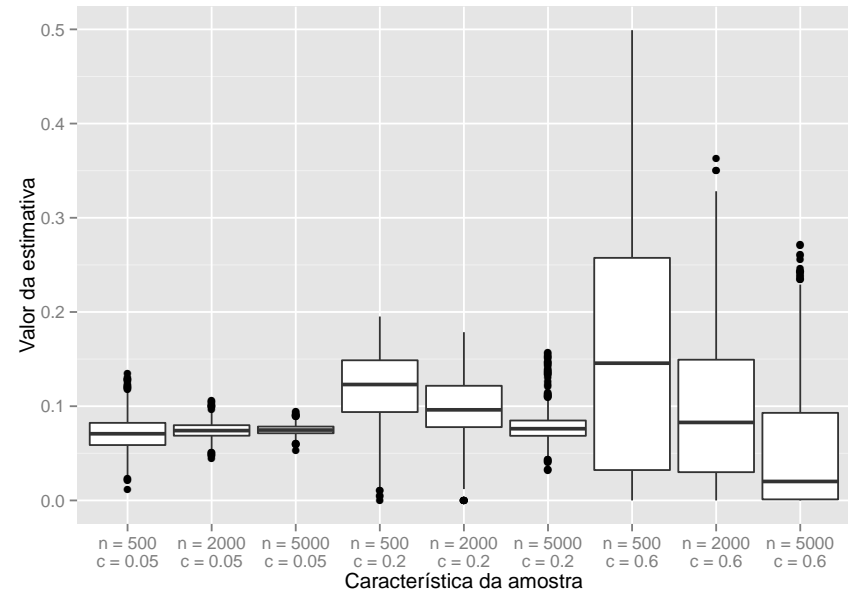

Figura B.21: cura segundo o modelo proposto.

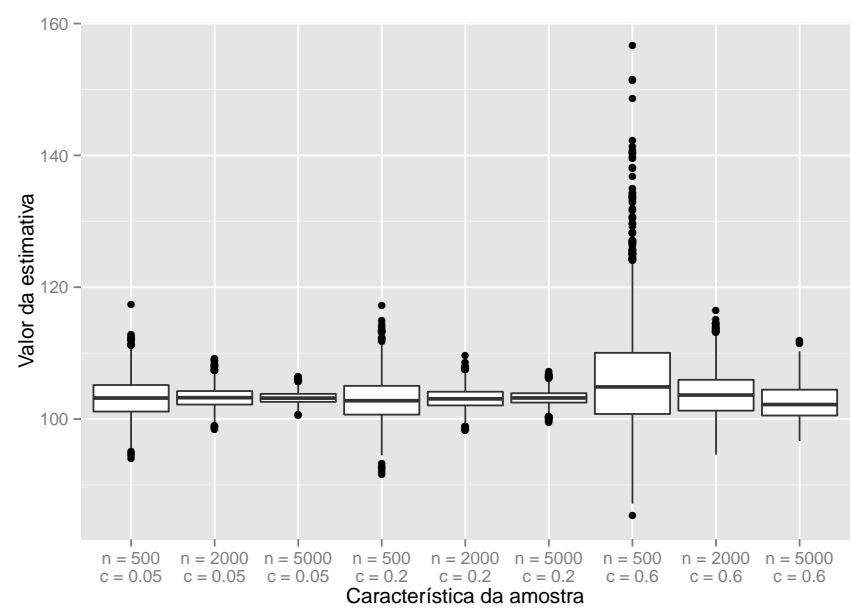

Figura B.23: $t_{\mathrm{Md}}$ segundo o modelo proposto.

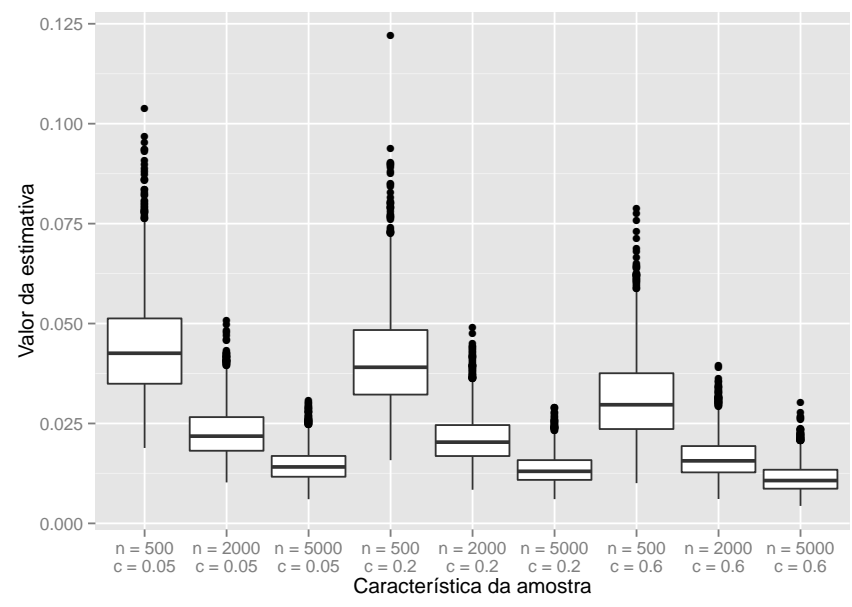

Figura B.25: $d_{\max }$ segundo o modelo proposto.

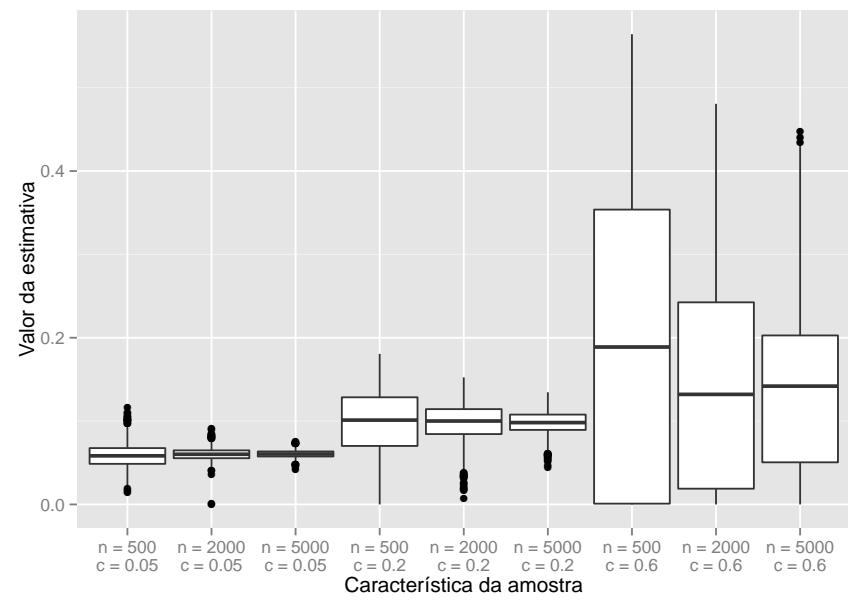

Figura B.22: cura segundo tempos de promoção.

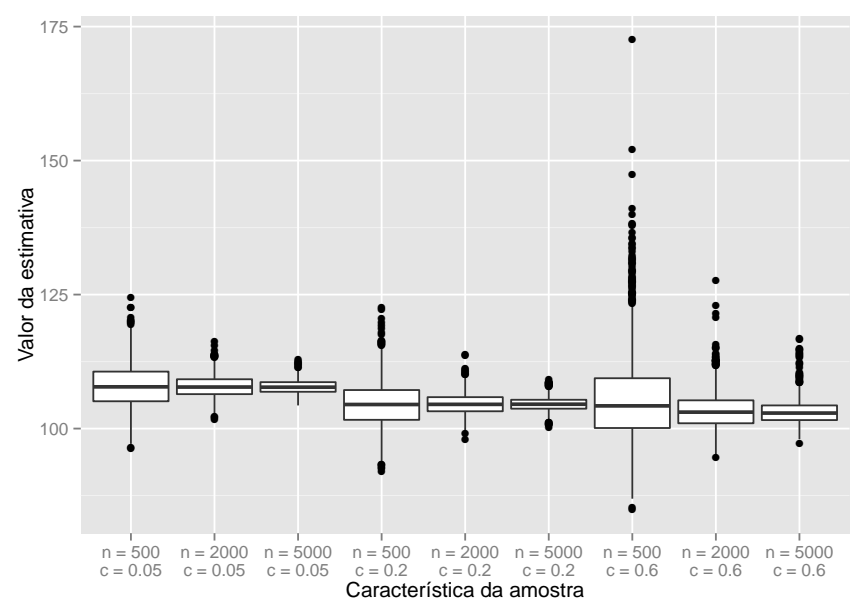

Figura B.24: $t_{\mathrm{Md}}$ segundo tempos de promoção.

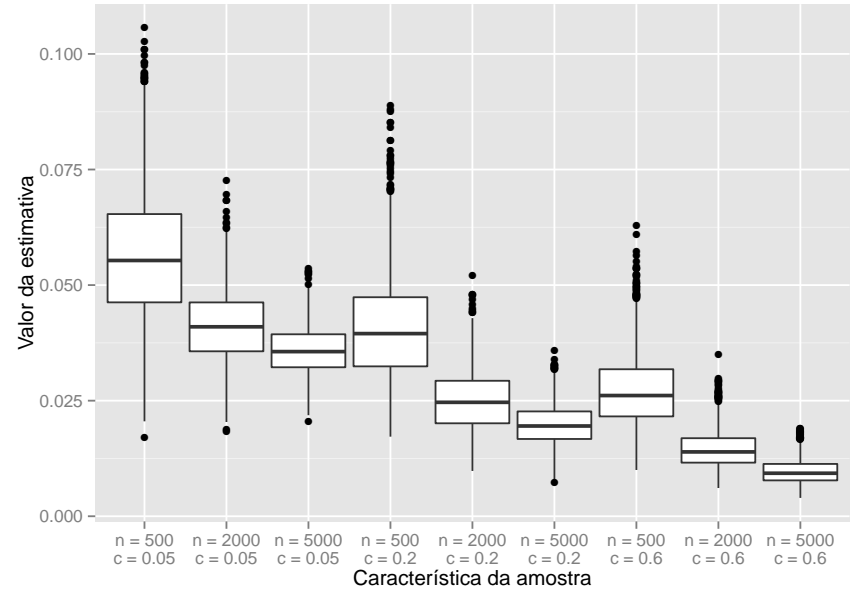

Figura B.26: $d_{\max }$ segundo tempos de promoção. 


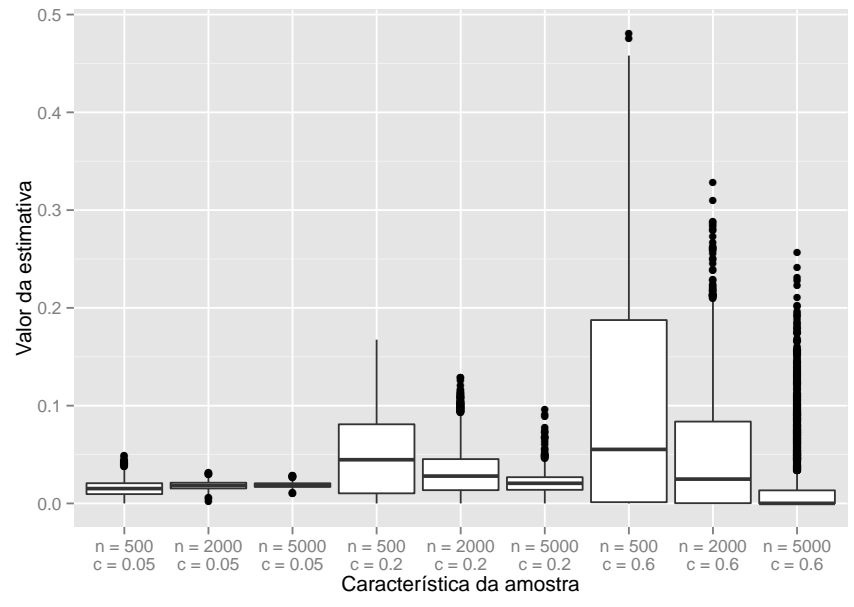

Figura B.27: cura segundo o modelo proposto.

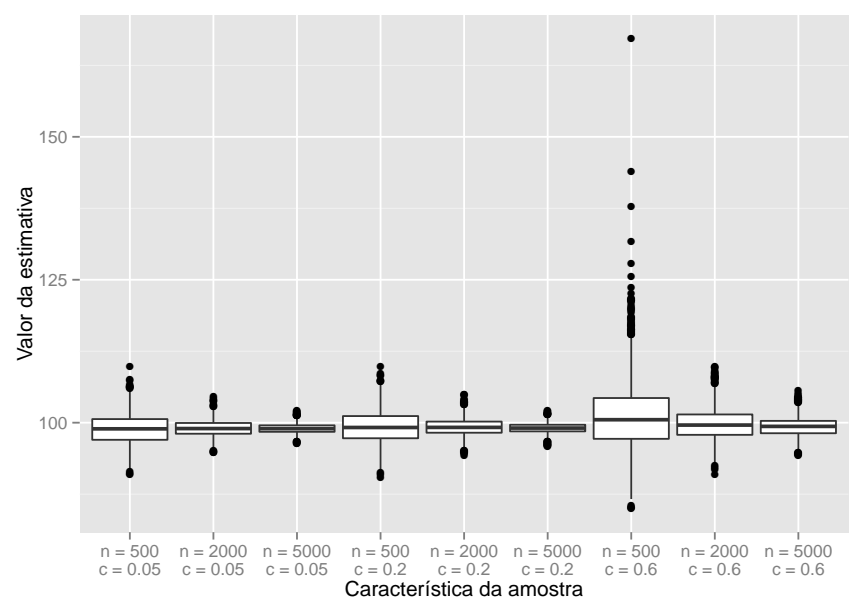

Figura B.29: $t_{\mathrm{Md}}$ segundo o modelo proposto.

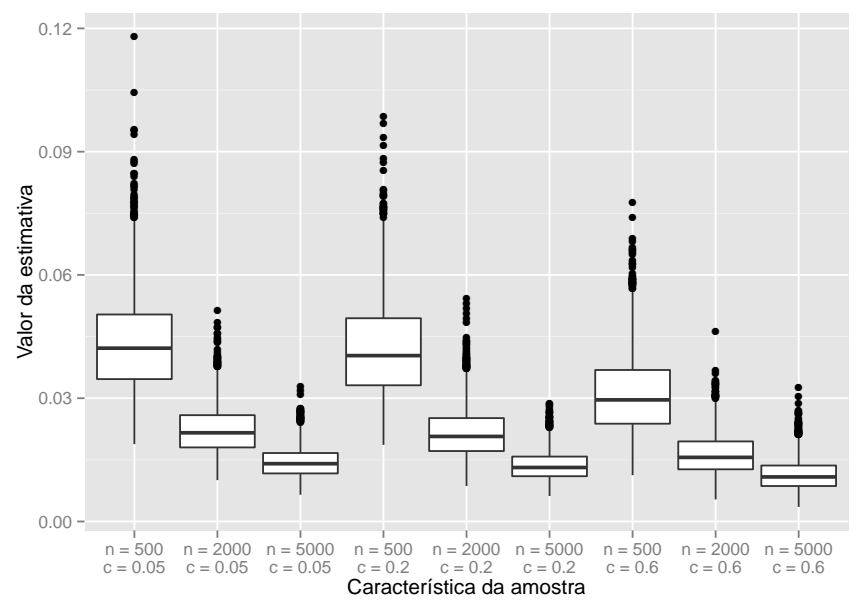

Figura B.31: $d_{\max }$ segundo o modelo proposto.

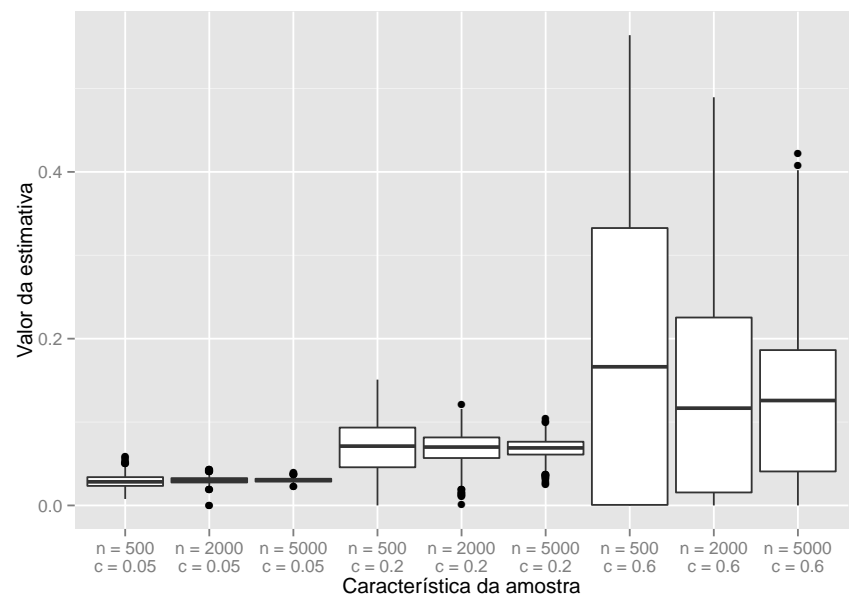

Figura B.28: cura segundo tempos de promoção.

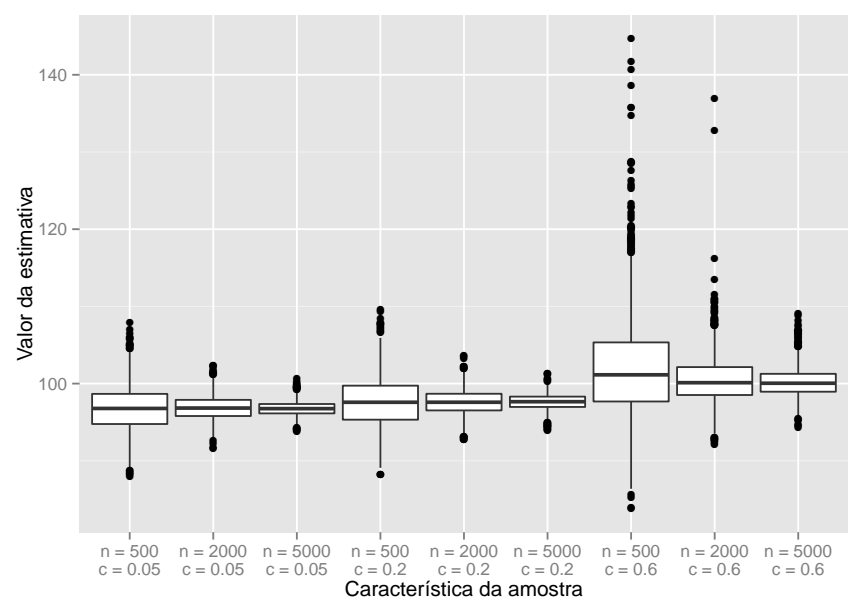

Figura B.30: $t_{\mathrm{Md}}$ segundo tempos de promoção.

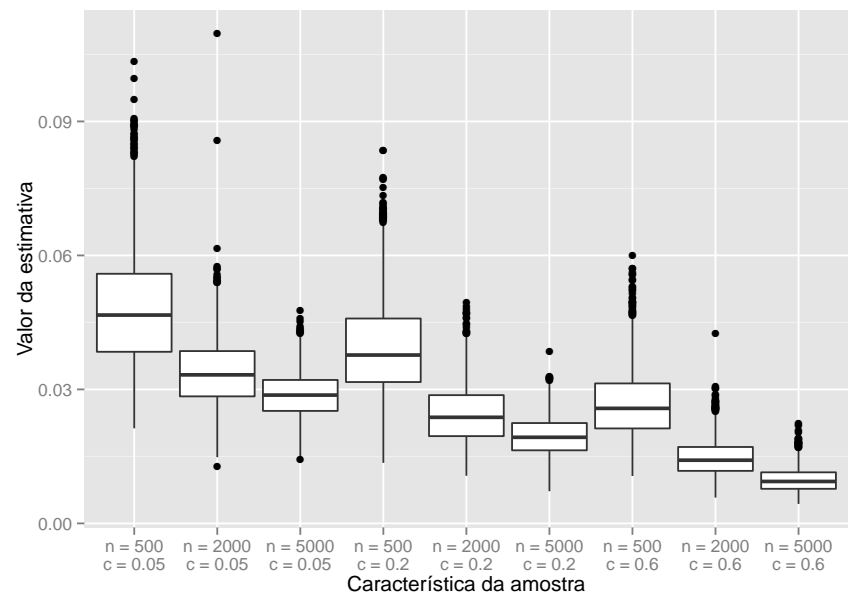

Figura B.32: $d_{\max }$ segundo tempos de promoção. 


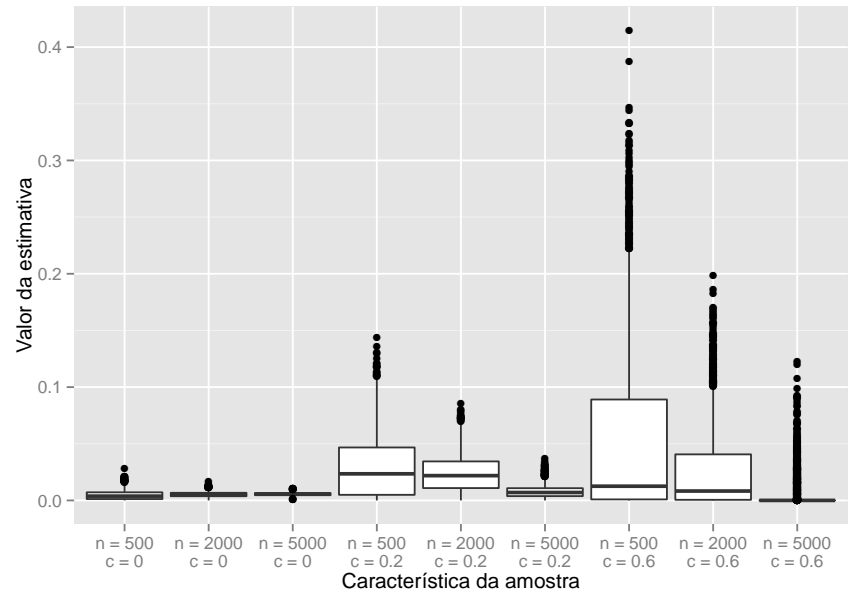

Figura B.33: cura segundo o modelo proposto.

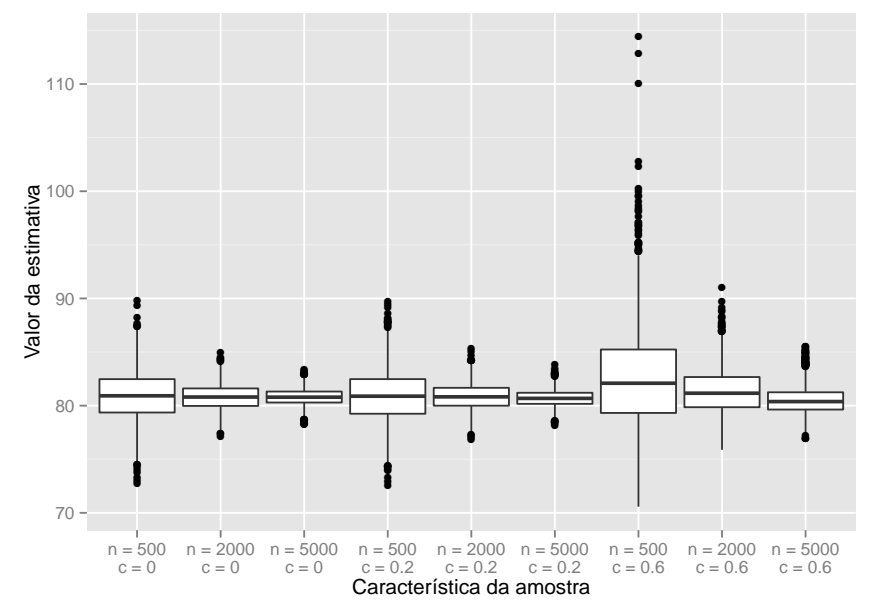

Figura B.35: $t_{\mathrm{Md}}$ segundo o modelo proposto.

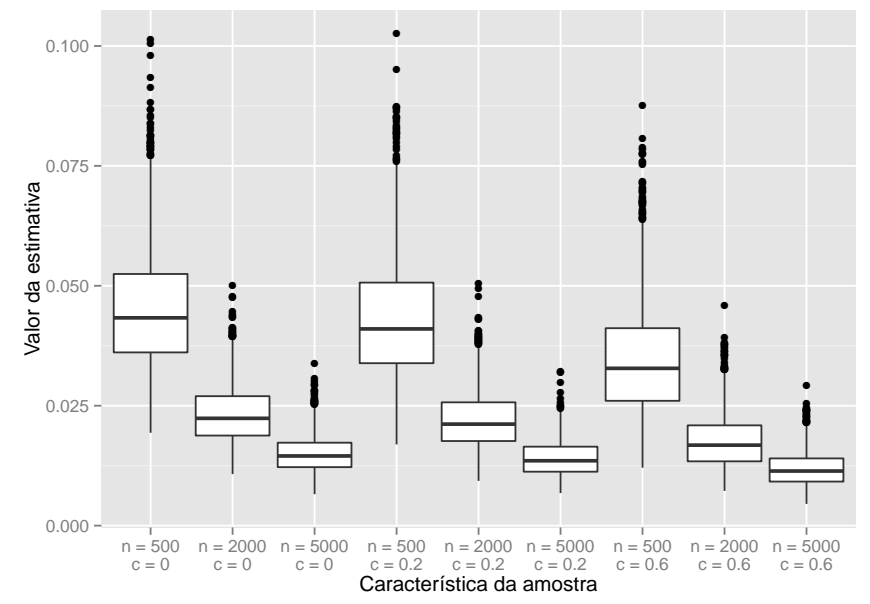

Figura B.37: $d_{\max }$ segundo o modelo proposto.

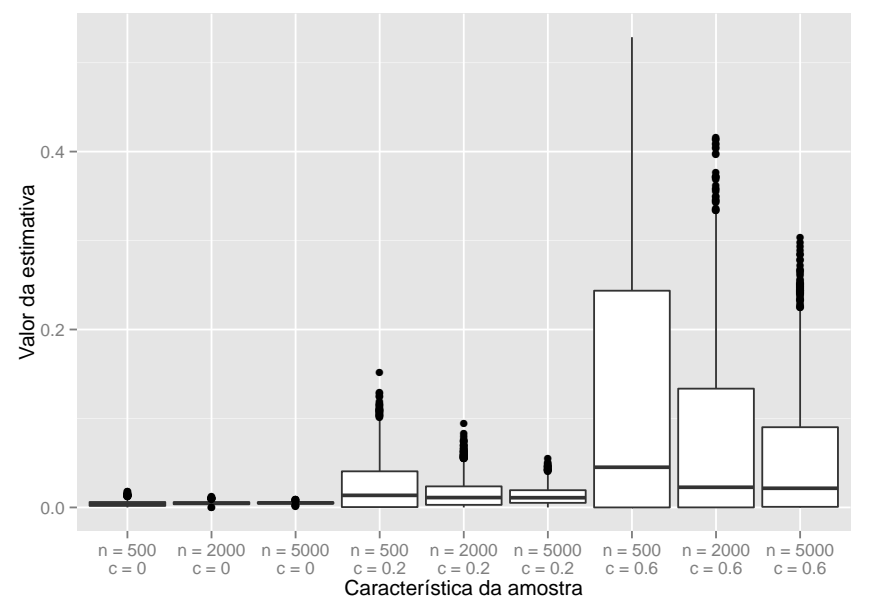

Figura B.34: cura segundo tempos de promoção.

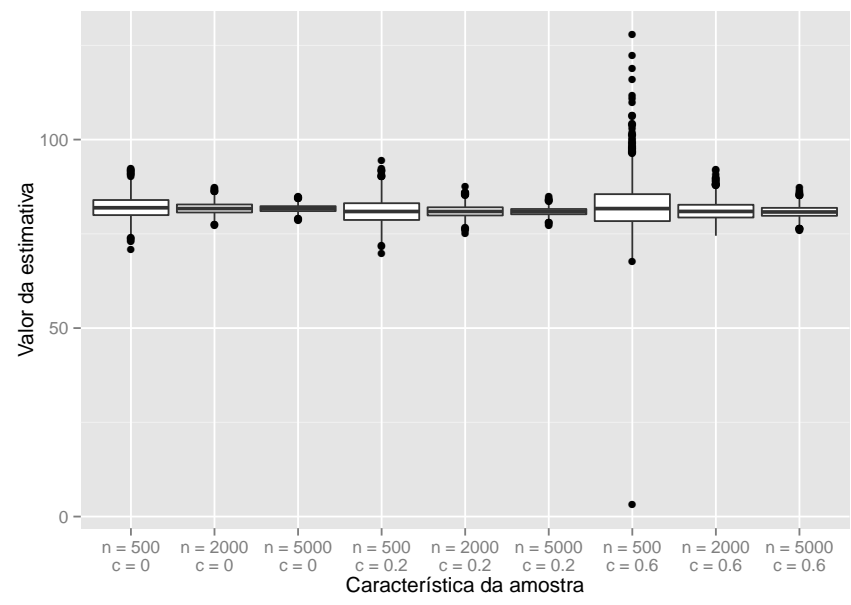

Figura B.36: $t_{\mathrm{Md}}$ segundo tempos de promoção.

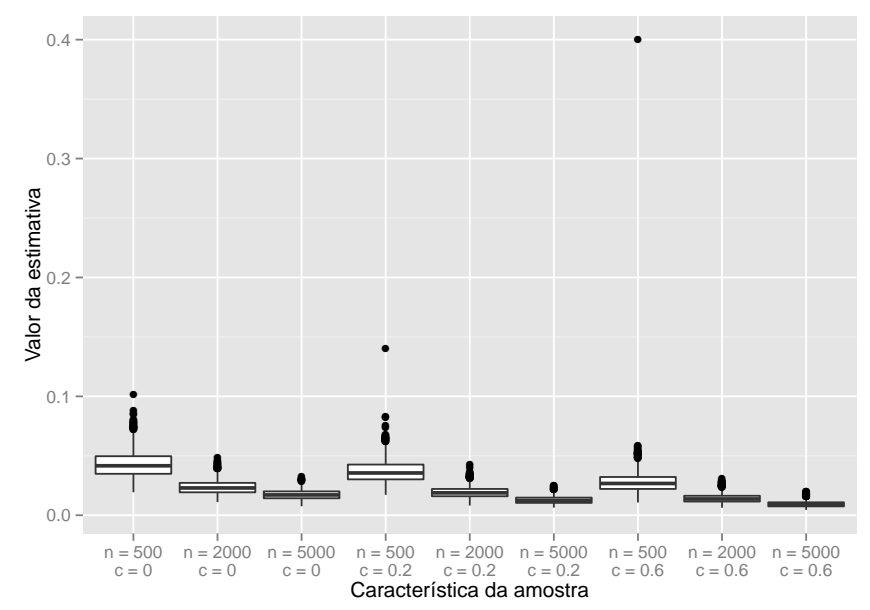

Figura B.38: $d_{\max }$ segundo tempos de promoção. 


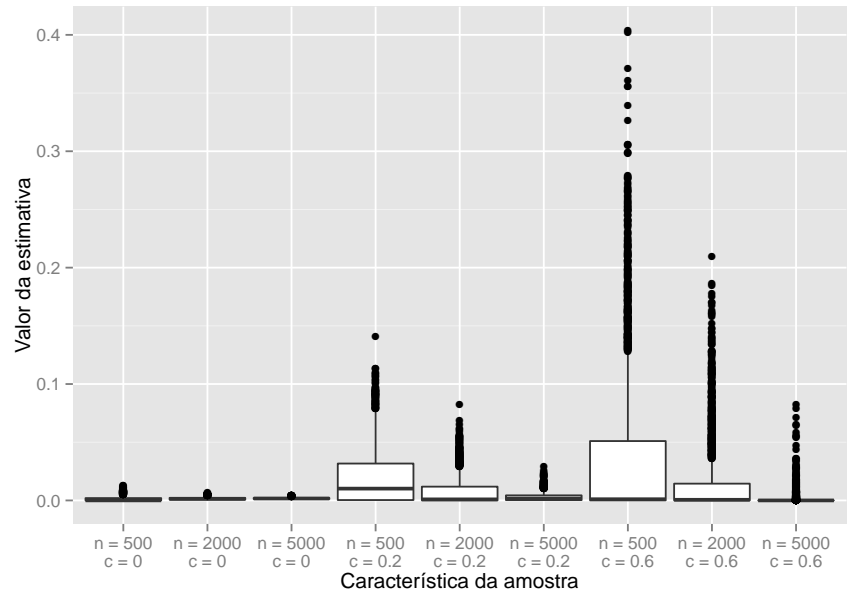

Figura B.39: cura segundo o modelo proposto.

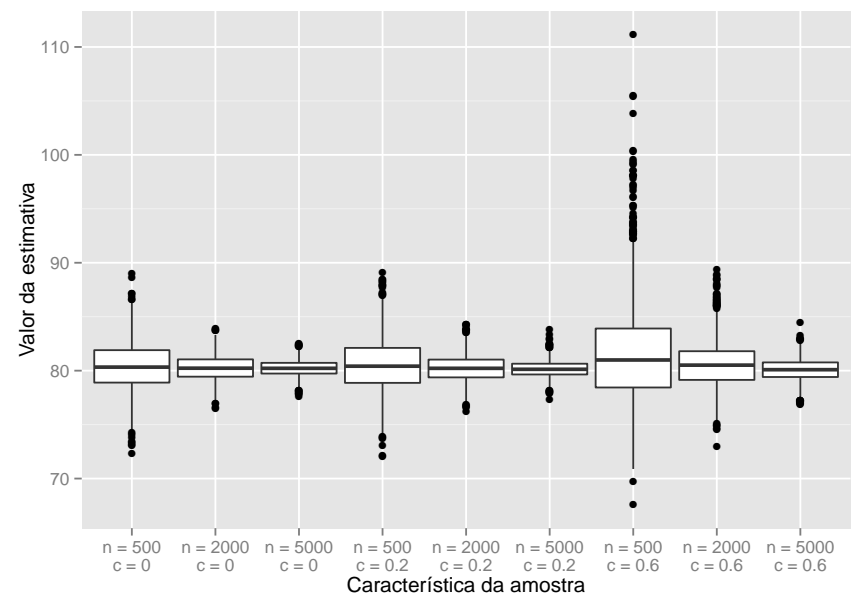

Figura B.41: $t_{\mathrm{Md}}$ segundo o modelo proposto.

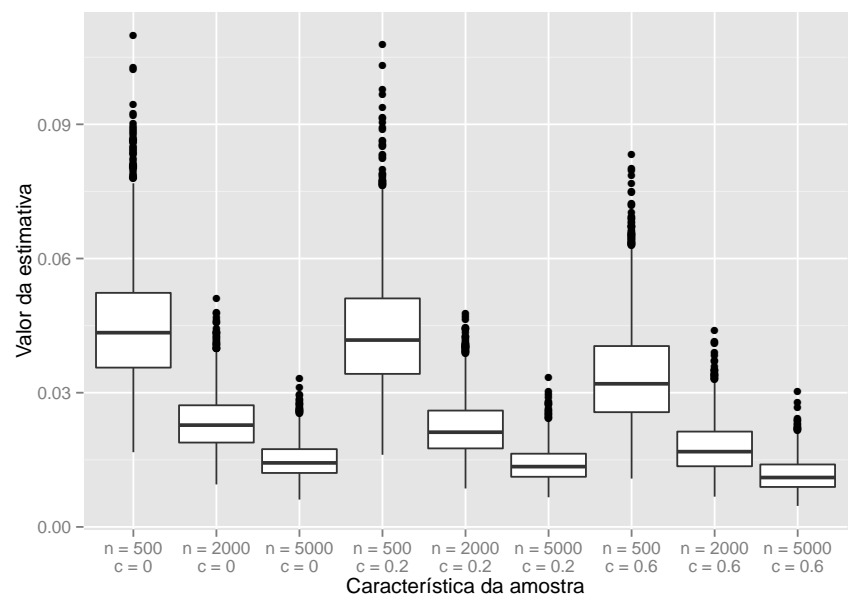

Figura B.43: $d_{\max }$ segundo o modelo proposto.

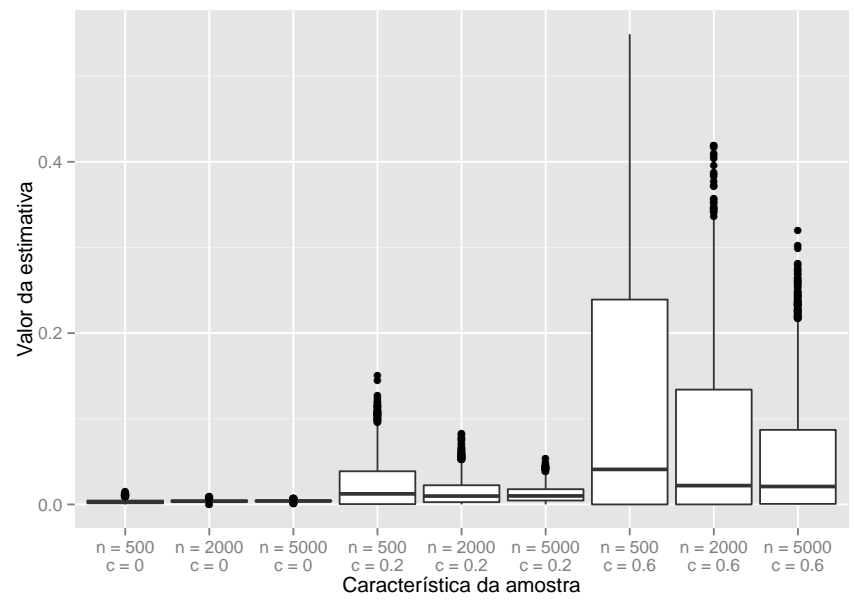

Figura B.40: cura segundo tempos de promoção.

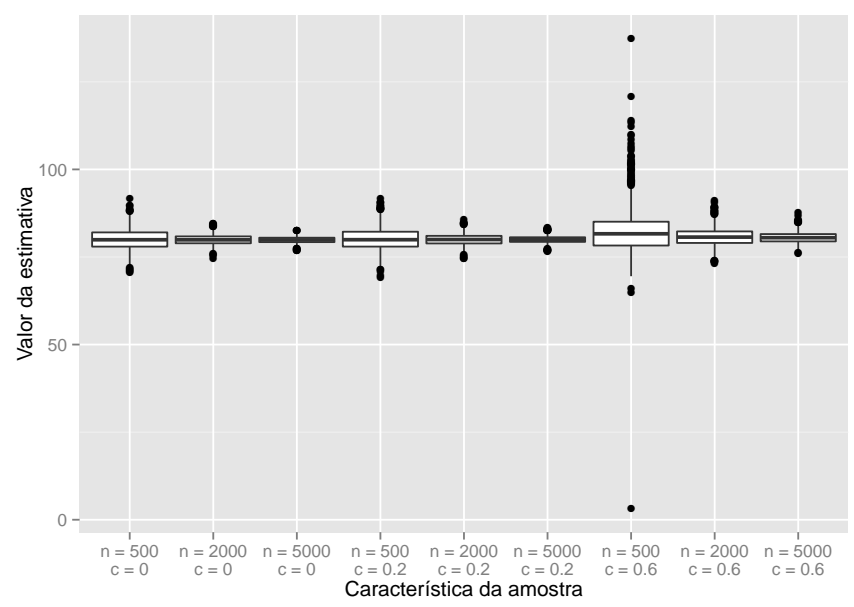

Figura B.42: $t_{\mathrm{Md}}$ segundo tempos de promoção.

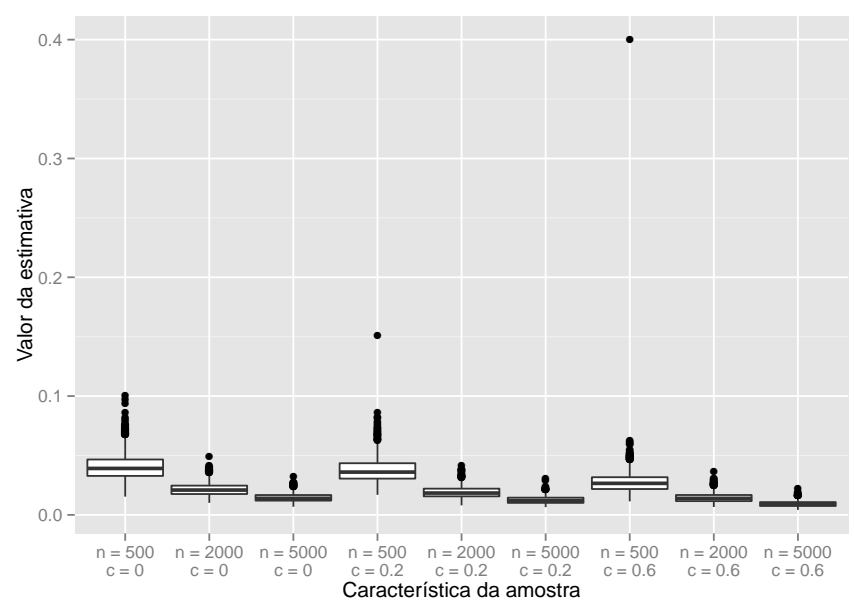

Figura B.44: $d_{\max }$ segundo tempos de promoção. 


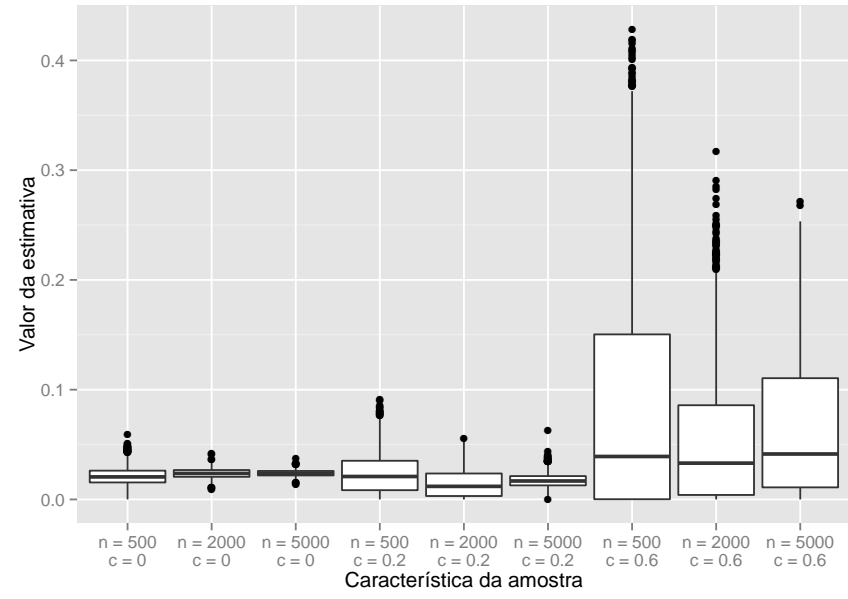

Figura B.45: cura segundo o modelo proposto.

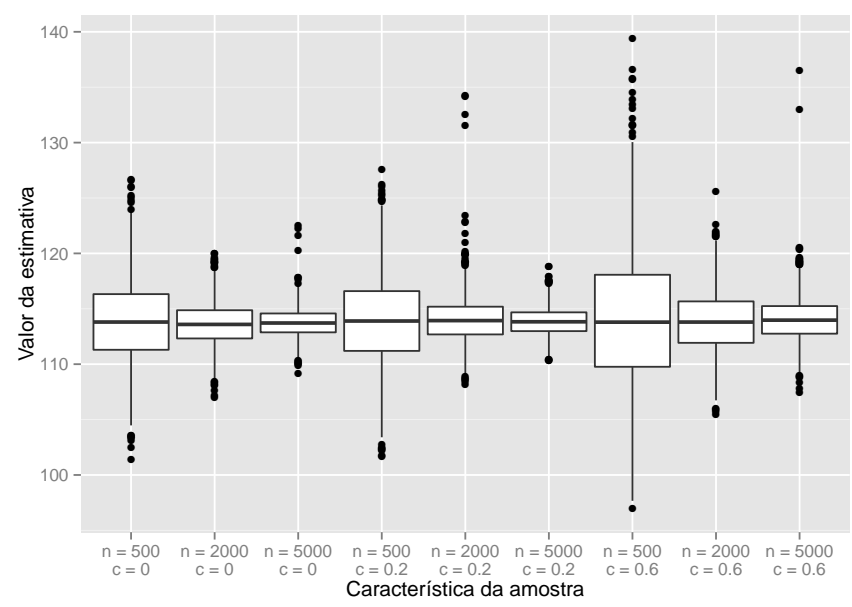

Figura B.47: $t_{\mathrm{Md}}$ segundo o modelo proposto.

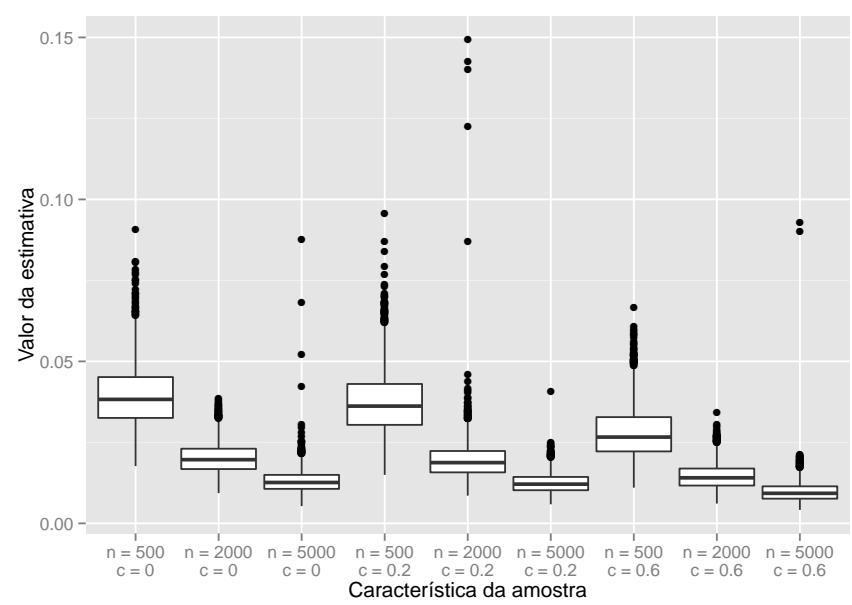

Figura B.49: $d_{\max }$ segundo o modelo proposto.

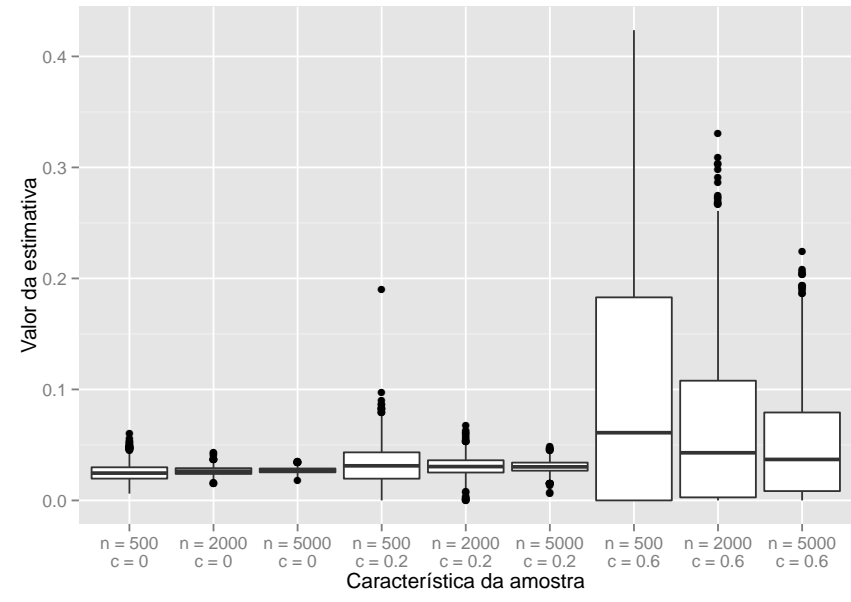

Figura B.46: cura segundo tempos de promoção.

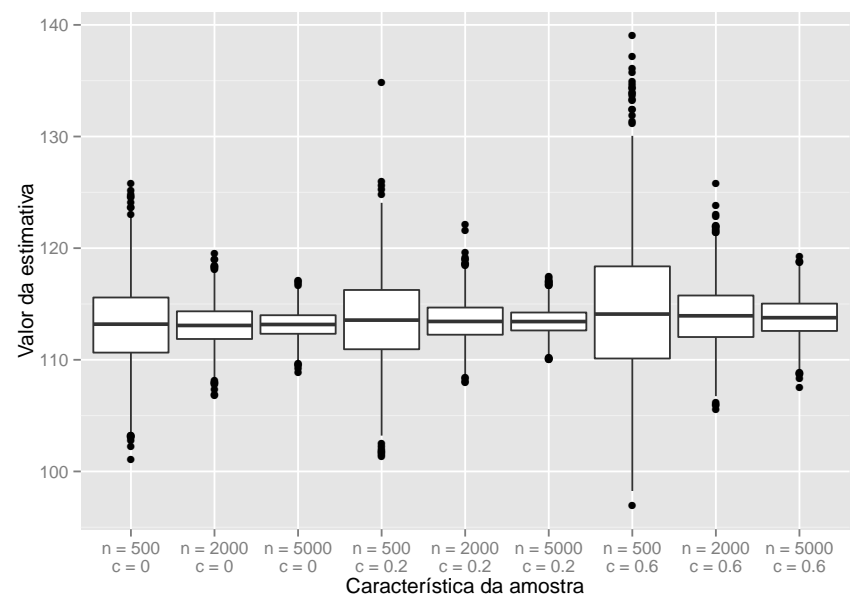

Figura B.48: $t_{\mathrm{Md}}$ segundo tempos de promoção.

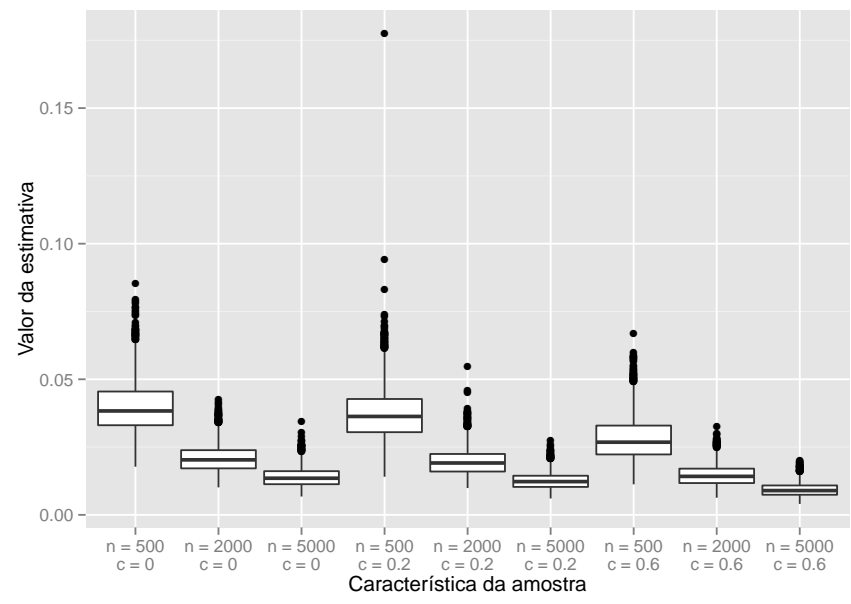

Figura B.50: $d_{\max }$ segundo tempos de promoção. 


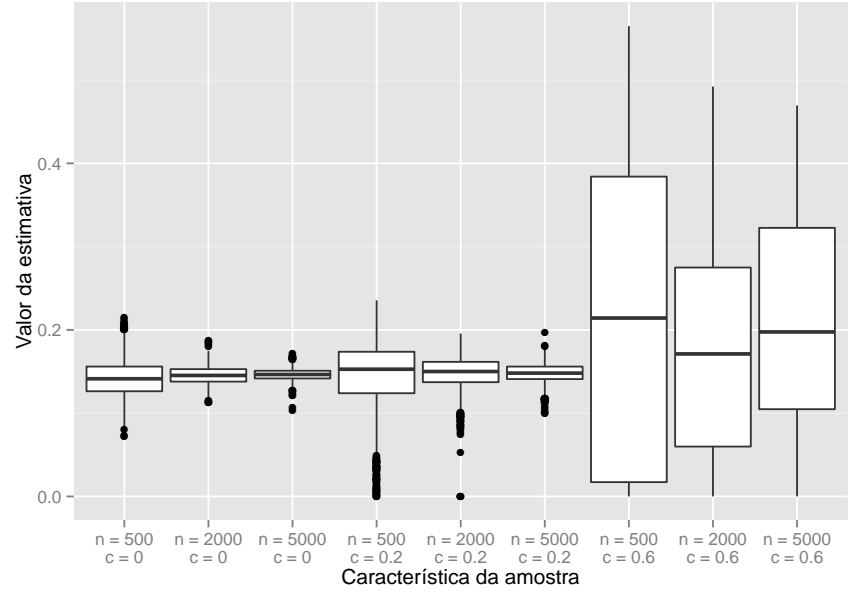

Figura B.51: cura segundo o modelo proposto.

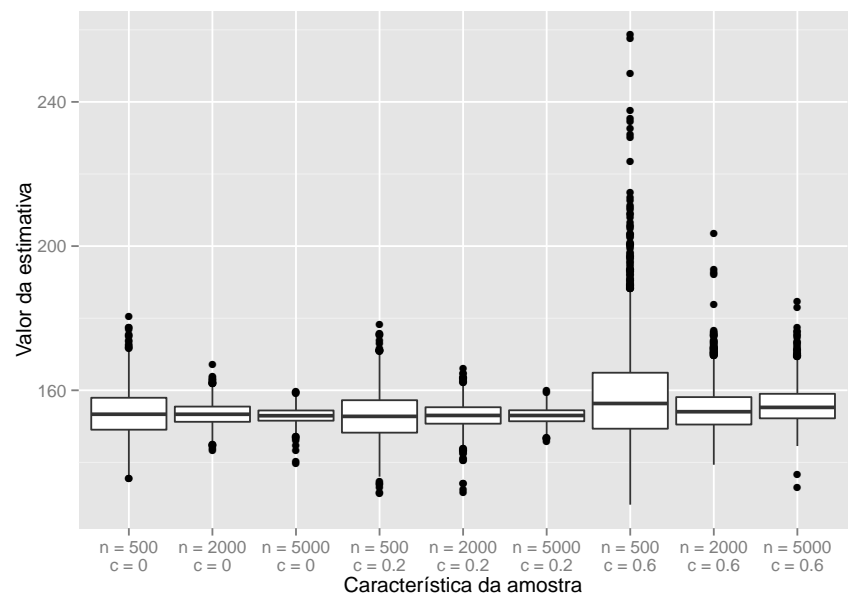

Figura B.53: $t_{\mathrm{Md}}$ segundo o modelo proposto.

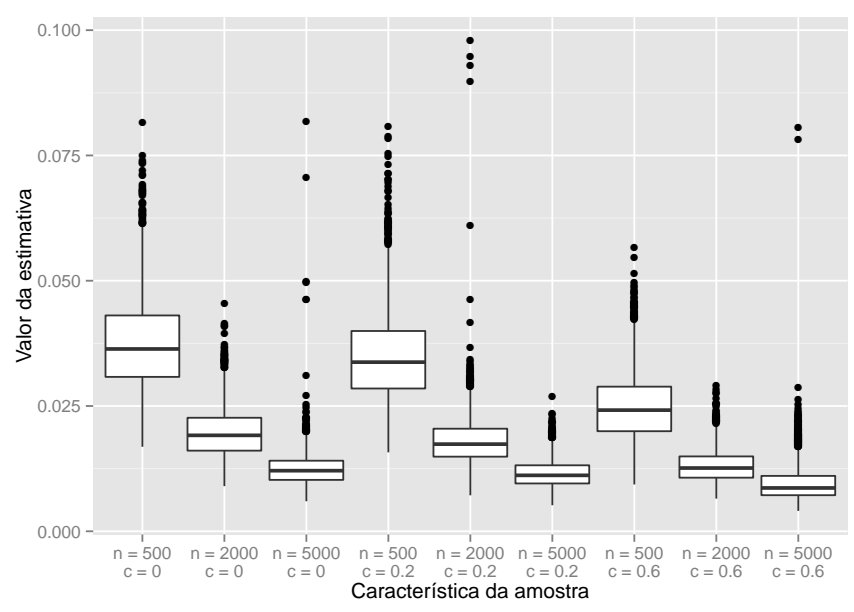

Figura B.55: $d_{\max }$ segundo o modelo proposto.

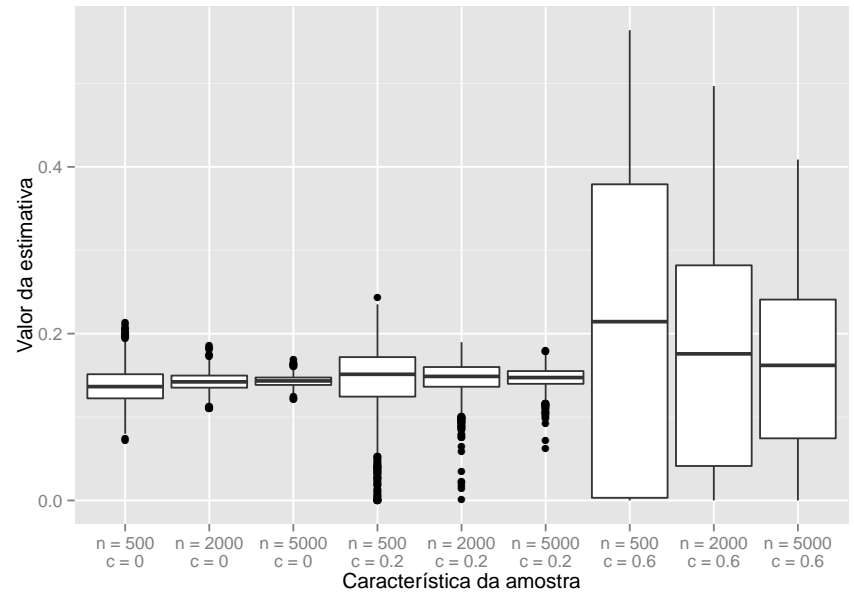

Figura B.52: cura segundo tempos de promoção.

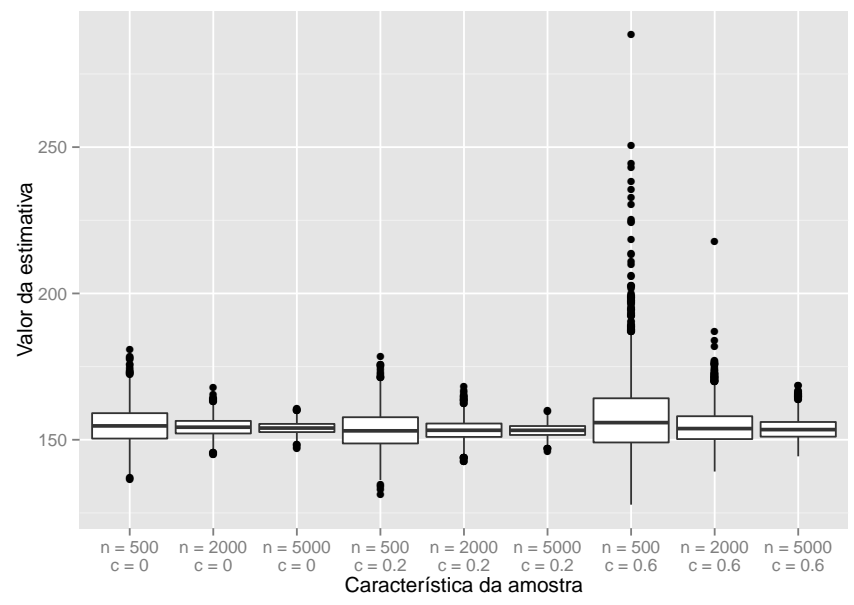

Figura B.54: $t_{\mathrm{Md}}$ segundo tempos de promoção.

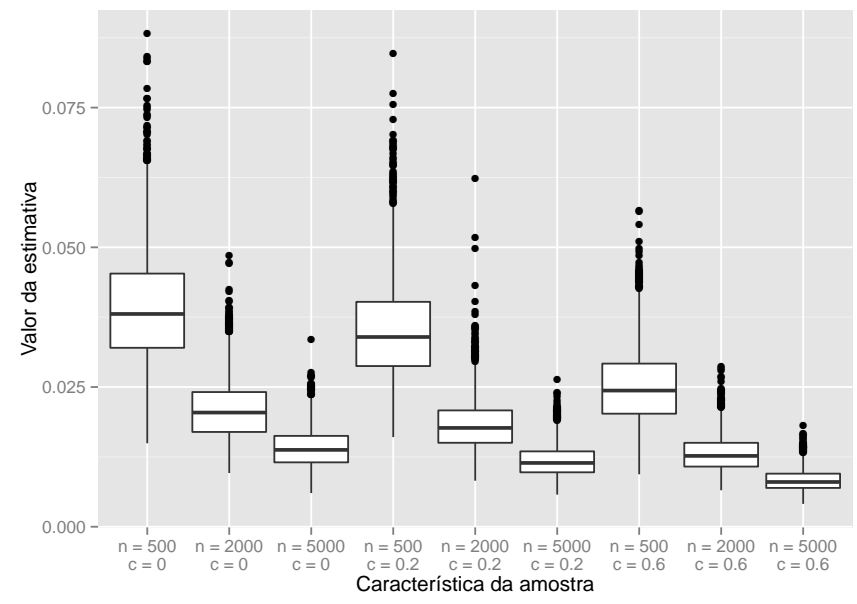

Figura B.56: $d_{\max }$ segundo tempos de promoção. 


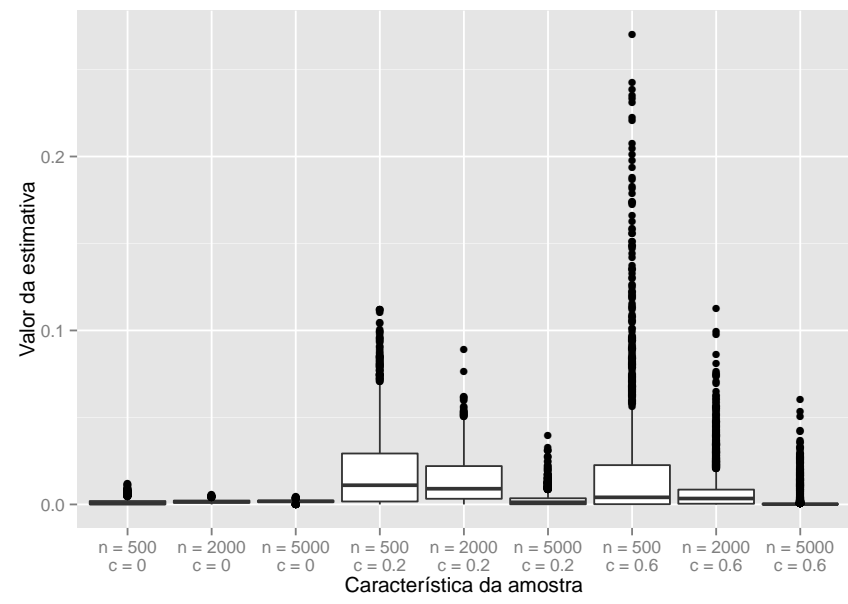

Figura B.57: cura segundo o modelo proposto.

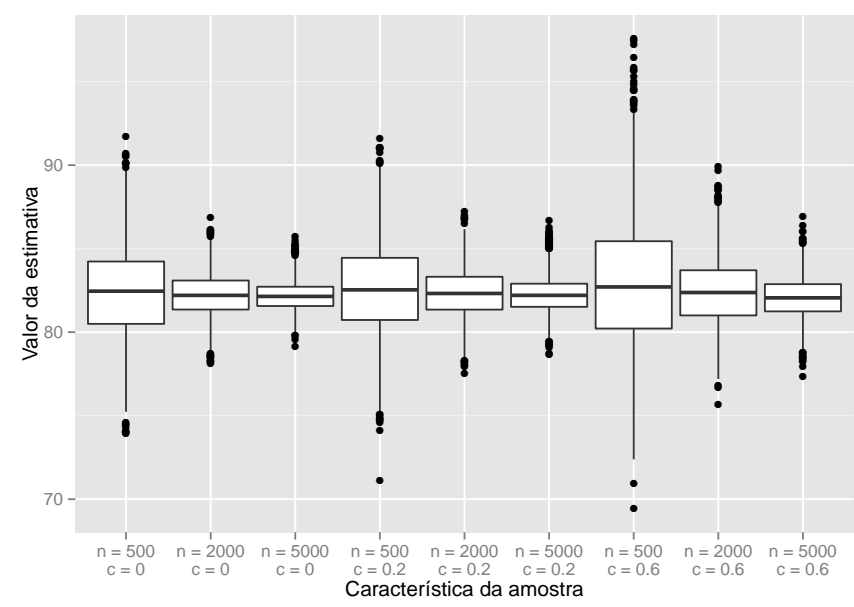

Figura B.59: $t_{\mathrm{Md}}$ segundo o modelo proposto.

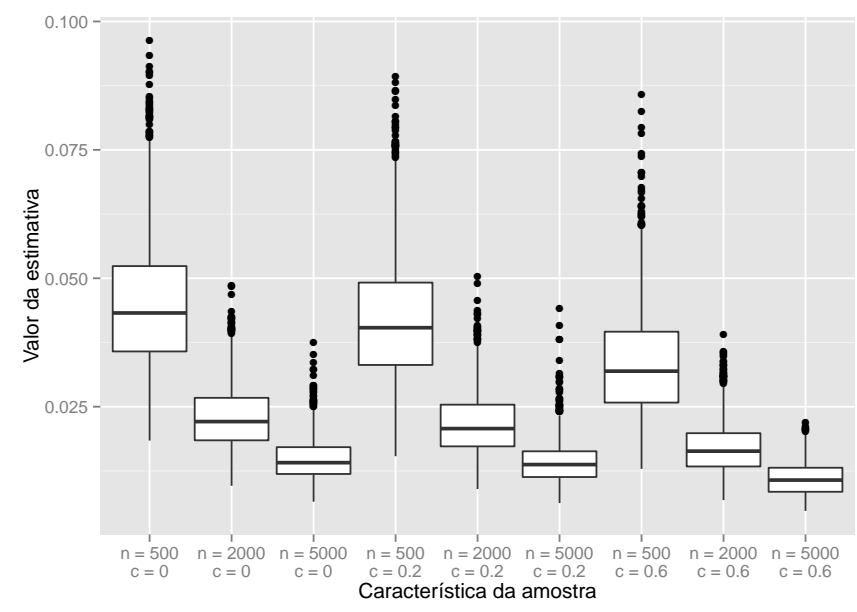

Figura B.61: $d_{\max }$ segundo o modelo proposto.

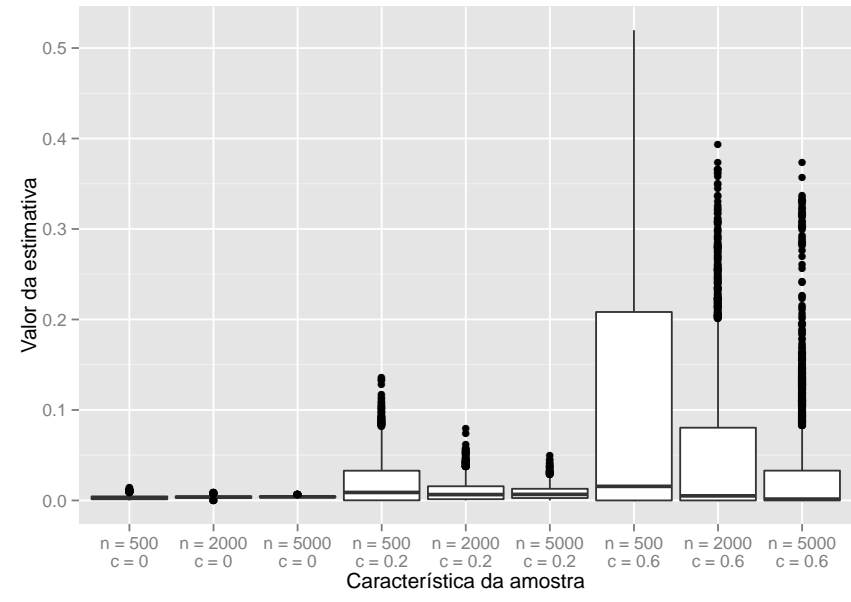

Figura B.58: cura segundo tempos de promoção.

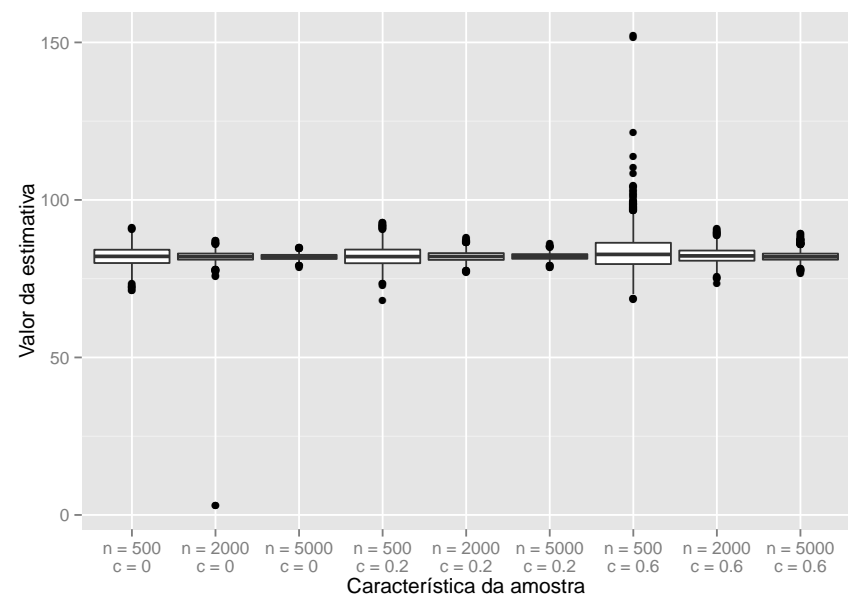

Figura B.60: $t_{\mathrm{Md}}$ segundo tempos de promoção.

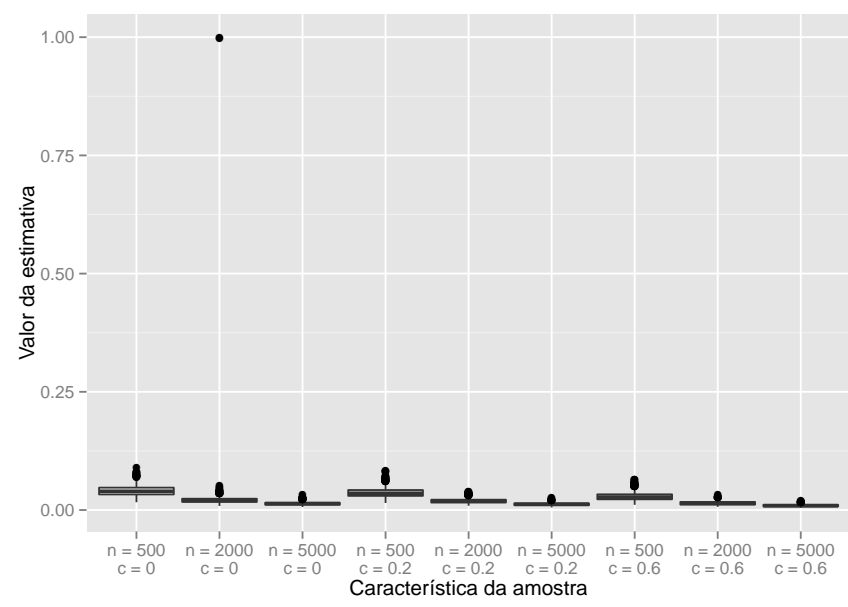

Figura B.62: $d_{\max }$ segundo tempos de promoção. 


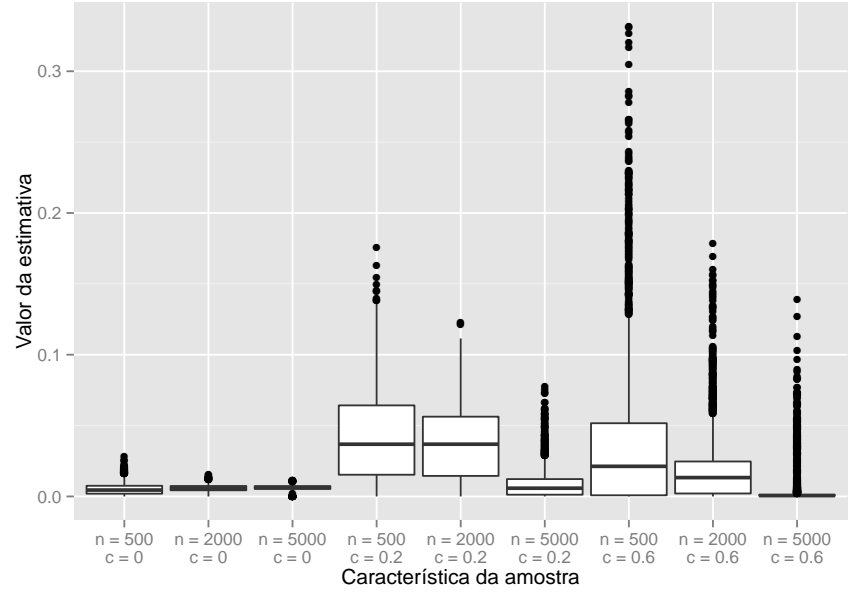

Figura B.63: cura segundo o modelo proposto.

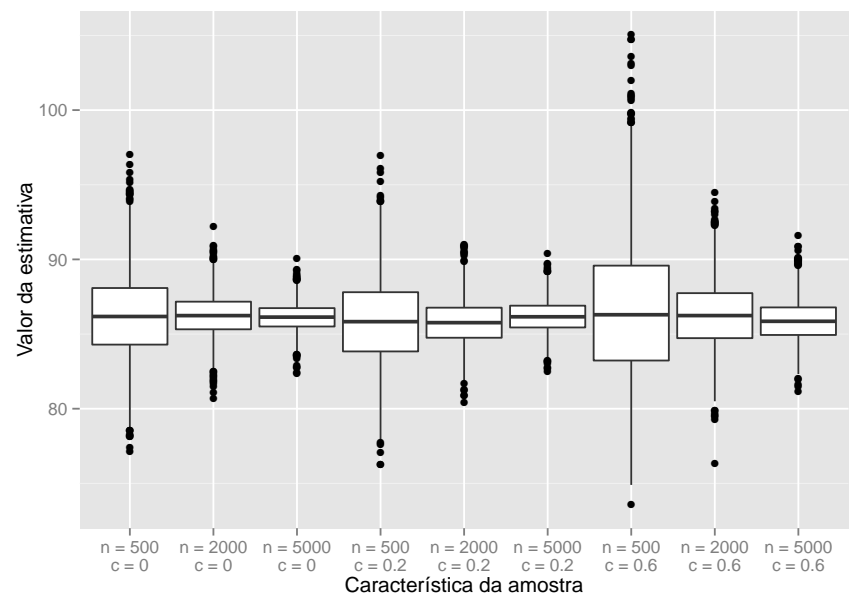

Figura B.65: $t_{\mathrm{Md}}$ segundo o modelo proposto.

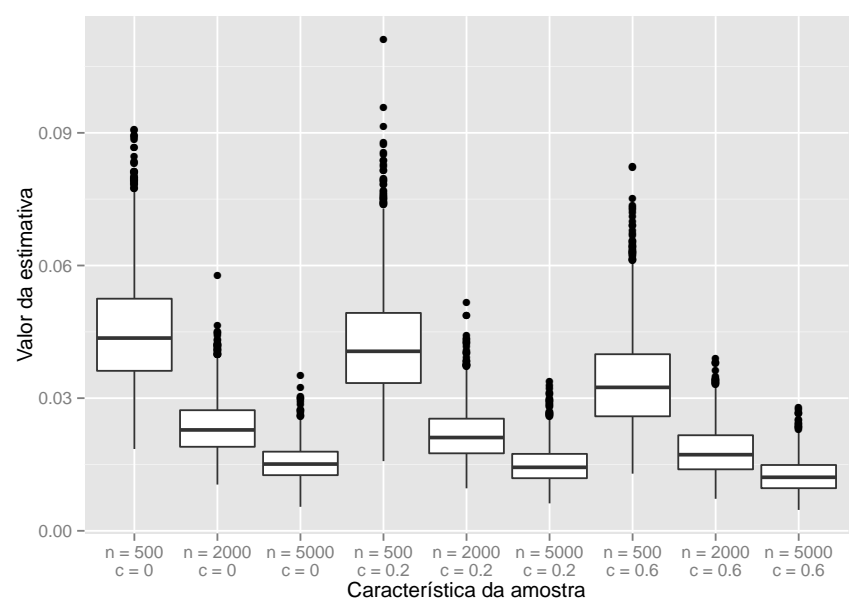

Figura B.67: $d_{\max }$ segundo o modelo proposto.

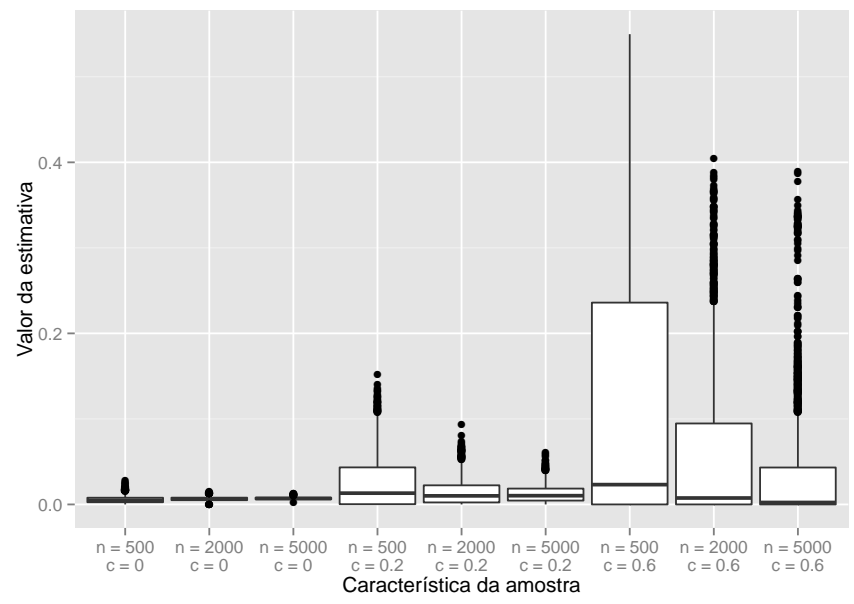

Figura B.64: cura segundo tempos de promoção.

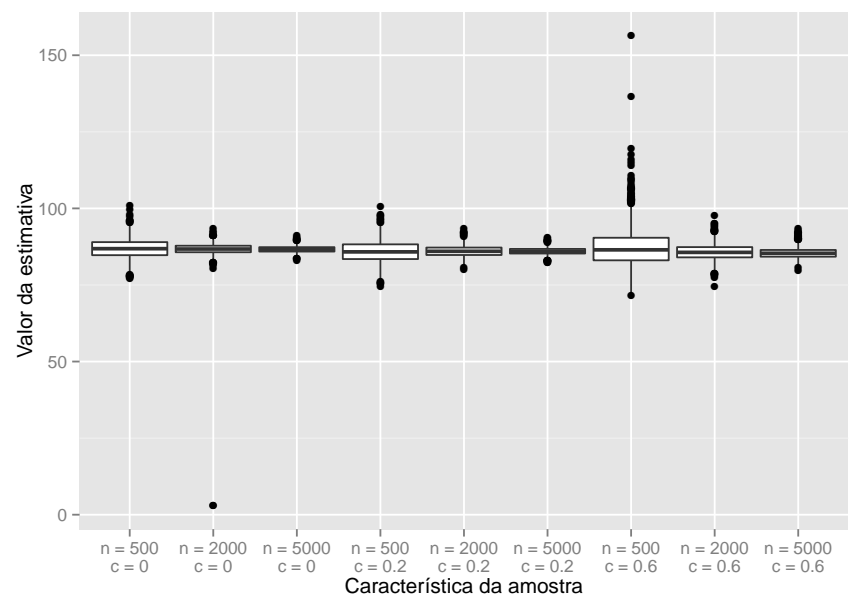

Figura B.66: $t_{\mathrm{Md}}$ segundo tempos de promoção.

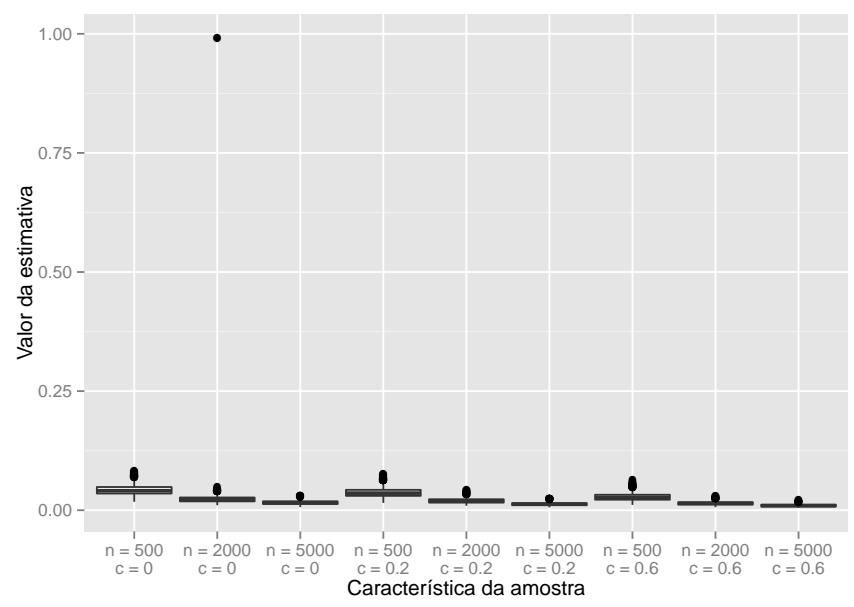

Figura B.68: $d_{\max }$ segundo tempos de promoção. 


\section{Referências Bibliográficas}

AKAIKE, H. (1974). A new look at the statistical model identification. Automatic Control, IEEE Transactions on 19, 716-723. (Citado na pág. 27.)

Armitage, P. \& Doll, R. (1954). The age distribution of cancer and a multi-stage theory of carcinogenesis. British journal of cancer 8, 1. (Citado na pág. 6.)

AtKinson, K. E. (2008). An introduction to numerical analysis. John Wiley \& Sons. (Citado na pág. 19.)

Berkson, J. \& Gage, R. (1952). Survival curve for cancer patients following treatment. Journal of the American Statistical Association , 501-515. (Citado nas págs. 1 e 7.)

BoAG, J. (1949). Maximum likelihood estimates of the proportion of patients cured by cancer therapy. Journal of the Royal Statistical Society. Series B (Methodological) 11, 15-53. (Citado na pág. 7.)

Chen, M., Ibrahim, J. \& Sinha, D. (1999). A new bayesian model for survival data with a surviving fraction. Journal of the American Statistical Association , 909-919. (Citado nas págs. 1 e 7.)

Chen, M.-H. \& Ibrahim, J. G. (2001). Maximum likelihood methods for cure rate models with missing covariates. Biometrics 57, 43-52. (Citado na pág. 16.)

ChiAng, C. L. (1980). An introduction to stochastic processes and their applications. Krieger. (Citado nas págs. 10 e 12.)

Cox, D. (1972). Regression models and life-tables. Journal of the Royal Statistical Society. Series B (Methodological) , 187-220. (Citado na pág. 7.)

Efron, B. (1979). Bootstrap methods: another look at the jackknife. The annals of Statistics , 1-26. (Citado na pág. 27.)

Henningsen, A. \& Toomet, O. (2011). maxlik: A package for maximum likelihood estimation in R. Computational Statistics 26, 443-458. (Citado na pág. 40.)

Hoggart, C. \& Griffin, J. (2001). A bayesian partition model for customer attrition. (Selected Papers from ISBA 2000): The Sixth World Meeting of the International Society for Bayesian Analysis , 223-232. (Citado na pág. 2.)

Ibrahim, J. G., Chen, M.-H. \& Sinha, D. (2001). Bayesian semiparametric models for survival data with a cure fraction. Biometrics 57, 383-388. (Citado na pág. 7.)

Kaplan, E. L. \& Meier, P. (1958). Nonparametric estimation from incomplete observations. Journal of the American statistical association 53, 457-481. (Citado na pág. 35.) 
Kim, S., Chen, M.-H., Dey, D. K. \& Gamerman, D. (2007). Bayesian dynamic models for survival data with a cure fraction. Lifetime Data Analysis 13, 17-35. (Citado na pág. 7.)

Klein, G. \& Klein, E. (1984). Oncogene activation and tumor progression. Carcinogenesis 5, 429-435. (Citado na pág. 6.)

Lambert, P. C., Thompson, J. R., Weston, C. L. \& Dickman, P. W. (2007). Estimating and modeling the cure fraction in population-based cancer survival analysis. Biostatistics 8, 576-594. (Citado na pág. 15.)

Moolgavkar, S. H., Dewanji, A. \& Venzon, D. J. (1988). A stochastic two-stage model for cancer risk assessment. i. the hazard function and the probability of tumor. Risk Analysis 8, 383-392. (Citado na pág. 6.)

Moolgavkar, S. H. \& Knudson, A. G. (1981). Mutation and cancer: a model for human carcinogenesis. Journal of the National Cancer Institute 66, 1037-1052. (Citado na pág. 6.)

Moolgavkar, S. H. \& Luebeck, E. G. (1992). Multistage carcinogenesis: population-based model for colon cancer. Journal of the National Cancer Institute 84, 610-618. (Citado na pág. 7.)

Moolgavkar, S. H. \& Venzon, D. J. (1979). Two-event models for carcinogenesis: incidence curves for childhood and adult tumors. Mathematical Biosciences 47, 55-77. (Citado na pág. 6.)

Peng, Y. (2003). Fitting semiparametric cure models. Computational statistics \& data analysis 41, 481-490. (Citado na pág. 7.)

Peng, Y. \& Dear, K. B. (2000). A nonparametric mixture model for cure rate estimation. Biometrics 56, 237-243. (Citado na pág. 7.)

Quenouille, M. H. (1949). Approximate tests of correlation in time-series. Journal of the Royal Statistical Society. Series B (Methodological) 11, 68-84. (Citado na pág. 47.)

R Core Team (2012). R: A Language and Environment for Statistical Computing. R Foundation for Statistical Computing, Vienna, Austria. Http://www.R-project.org/. (Citado na pág. 40.)

Robert, C. P. \& Casella, G. (2004). Monte Carlo statistical methods, vol. 319. Citeseer. (Citado na pág. 40.)

Rodrigues, J., Cancho, V., de Castro, M. \& Louzada-Neto, F. (2009a). On the unification of long-term survival models. Statistics \& Probability Letters 79, 753-759. (Citado na pág. 1.)

Rodrigues, J., De Castro, M., Balakrishnan, N. \& Cancho, V. (2011). Destructive weighted poisson cure rate models. Lifetime data analysis 17, 333-346. (Citado nas págs. 1 e 2.)

Rodrigues, J., de Castro, M., Cancho, V. G. \& Balakrishnan, N. (2009b). Com-poisson cure rate survival models and an application to a cutaneous melanoma data. Journal of Statistical Planning and Inference 139, 3605-3611. (Citado na pág. 1.)

Schwarz, G. (1978). Estimating the dimension of a model. The annals of statistics 6, 461-464. (Citado na pág. 27.)

SHAO, J. \& Tu, D. (1995). The jackknife and bootstrap. Springer. (Citado na pág. 47.)

Sposto, R. (2002). Cure model analysis in cancer: an application to data from the children's cancer group. Statistics in medicine 21, 293-312. (Citado nas págs. 15 e 16.) 
TAN, W. \& Chen, C. (1995). A bivariate stochastic model of carcinogenesis involving two cancer tumors. In the Proceeding of the 9 th International Conference on Mathematical and Computer Modelling. (Citado na pág. 6.)

TAN, W. \& Chen, C. (1998). Stochastic modeling of carcinogenesis: some new insights. Mathematical and computer modelling $\mathbf{2 8}, \mathbf{4 9 - 7 1 . ~ ( C i t a d o ~ n a ~ p a ́ g . ~ 6 . ) ~}$

TAN, W. \& Singh, K. P. (1987). Assessing the effects of metabolism of environmental agents on cancer tumor development by a two-stage model of carcinogenesis. Environmental health perspectives 74, 203. (Citado na pág. 6.)

TAN, W.-Y. (1991). Stochastic Models for Carcinogenesis, vol. 116. CRC Press. (Citado na pág. 6.)

TAN, W. Y. (2002). Stochastic Models With Applications to Genetics, Cancers, AIDS And Other Biomemedical Systems, vol. 4 of Series on Concrete and Applicable Mathematics. World Scientific. (Citado na pág. 5.)

Tournoud, M. \& Ecochard, R. (2007). Application of the promotion time cure model with time-changing exposure to the study of hiv/aids and other infectious diseases. Statistics in medicine 26, 1008-1021. (Citado nas págs. 1 e 2.)

Tsodikov, A., Ibrahim, J. \& Yakovlev, A. (2003). Estimating cure rates from survival data. Journal of the American Statistical Association 98, 1063-1078. (Citado nas págs. 1 e 5.)

Yakovlev, A., Tsodikov, A. \& BAss, L. (1993). A stochastic model of hormesis. Mathematical biosciences 116, 197-219. (Citado nas págs. 1, 3 e 7.)

YAKovlev, A. Y. \& Tsodikov, A. D. (1996). Stochastic models of tumor latency and their biostatistical applications, vol. 1 of Series in Mathematical Biology and Medicine. World Scientific. (Citado nas págs. 4 e 5.)

YANG, G. L. \& Chen, C. W. (1991). A stochastic two-stage carcinogenesis model: a new approach to computing the probability of observing tumor in animal bioassays. Mathematical biosciences 104, 247-258. (Citado na pág. 6.)

Yin, G. \& Ibrahim, J. G. (2005). A general class of bayesian survival models with zero and nonzero cure fractions. Biometrics 61, 403-412. (Citado na pág. 7.) 